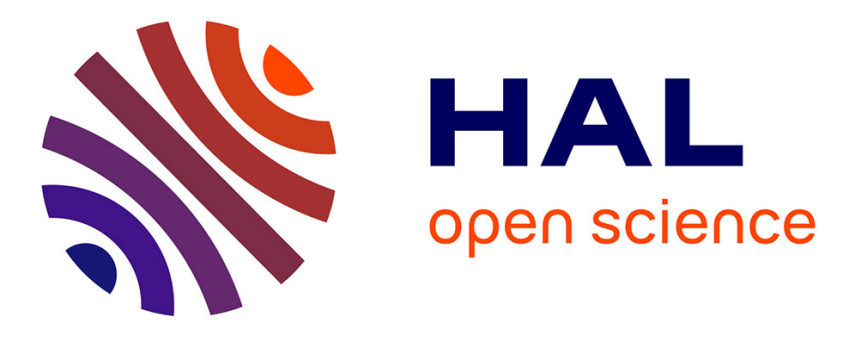

\title{
The Sample Analysis at Mars Investigation and Instrument Suite
}

Paul R. Mahaffy, Christopher R. Webster, Michel Cabane, Pamela G. Conrad, Patrice Coll, Sushil K. Atreya, Robert Arvey, Michael Barciniak, Mehdi Benna, Lora Bleacher, et al.

\section{To cite this version:}

Paul R. Mahaffy, Christopher R. Webster, Michel Cabane, Pamela G. Conrad, Patrice Coll, et al.. The Sample Analysis at Mars Investigation and Instrument Suite. Space Science Reviews, 2012, 170 (1-4), pp.401-478. 10.1007/s11214-012-9879-z . hal-00694758

\section{HAL Id: hal-00694758 https://hal.science/hal-00694758}

Submitted on 14 Jan 2020

HAL is a multi-disciplinary open access archive for the deposit and dissemination of scientific research documents, whether they are published or not. The documents may come from teaching and research institutions in France or abroad, or from public or private research centers.
L'archive ouverte pluridisciplinaire $\mathbf{H A L}$, est destinée au dépôt et à la diffusion de documents scientifiques de niveau recherche, publiés ou non, émanant des établissements d'enseignement et de recherche français ou étrangers, des laboratoires publics ou privés. 


\section{The Sample Analysis at Mars Investigation and Instrument Suite}

Paul R. Mahaffy • Christopher R. Webster • Michel Cabane • Pamela G. Conrad • Patrice Coll - Sushil K. Atreya - Robert Arvey - Michael Barciniak • Mehdi Benna • Lora Bleacher • William B. Brinckerhoff • Jennifer L. Eigenbrode · Daniel Carignan • Mark Cascia • Robert A. Chalmers · Jason P. Dworkin - Therese Errigo • Paula Everson · Heather Franz • Rodger Farley • Steven Feng • Gregory Frazier • Caroline Freissinet • Daniel P. Glavin • Daniel N. Harpold • Douglas Hawk • Vincent Holmes • Christopher S. Johnson • Andrea Jones • Patrick Jordan • James Kellogg • Jesse Lewis • Eric Lyness • Charles A. Malespin • David K. Martin • John Maurer • Amy C. McAdam • Douglas McLennan • Thomas J. Nolan • Marvin Noriega - Alexander A. Pavlov • Benito Prats • Eric Raaen • Oren Sheinman • David Sheppard · James Smith · Jennifer C. Stern · Florence Tan • Melissa Trainer • Douglas W. Ming • Richard V. Morris · John Jones • Cindy Gundersen • Andrew Steele · James Wray • Oliver Botta - Laurie A. Leshin - Tobias Owen • Steve Battel • Bruce M. Jakosky • Heidi Manning • Steven Squyres • Rafael Navarro-González • Christopher P. McKay • Francois Raulin • Robert Sternberg • Arnaud Buch • Paul Sorensen • Robert Kline-Schoder • David Coscia - Cyril Szopa · Samuel Teinturier • Curt Baffes · Jason Feldman • Greg Flesch • Siamak Forouhar • Ray Garcia • Didier Keymeulen - Steve Woodward • Bruce P. Block • Ken Arnett • Ryan Miller • Charles Edmonson • Stephen Gorevan • Erik Mumm

P.R. Mahaffy $(\varangle)$ · P.G. Conrad · R. Arvey · M. Barciniak · M. Benna · L. Bleacher · W.B. Brinckerhoff · J.L. Eigenbrode - D. Carignan · M. Cascia · R.A. Chalmers · J.P. Dworkin · T. Errigo · P. Everson - H. Franz - R. Farley · S. Feng · G. Frazier · C. Freissinet · D.P. Glavin · D.N. Harpold - D. Hawk · V. Holmes · C.S. Johnson - A. Jones · P. Jordan - J. Kellogg · J. Lewis · E. Lyness · C.A. Malespin - D.K. Martin · J. Maurer · A.C. McAdam · D. McLennan · T.J. Nolan · M. Noriega · A.A. Pavlov · B. Prats · E. Raaen · O. Sheinman · D. Sheppard · J. Smith · J.C. Stern · F. Tan $\cdot$ M. Trainer NASA Goddard Space Flight Center, 8800 Greenbelt Rd., Greenbelt, MD 20771, USA e-mail: Paul.R.Mahaffy@nasa.gov

C.R. Webster · C. Baffes · J. Feldman · G. Flesch · S. Forouhar · R. Garcia · D. Keymeulen · S. Woodward Jet Propulsion Laboratory, Pasadena, CA 91109, USA 
P. Coll

Denis Diderot et CNRS, Membre de l'Institut Universitaire de France, CMC, LISA, Universités Paris Est-Créteil, Paris 7, 61 av. du Général de Gaulle, 94010 Créteil cedex, France

\section{S.K. Atreya}

Department of Atmospheric, Oceanic, and Space Sciences, University of Michigan, Ann Arbor, MI 48109-2143, USA

D.W. Ming · R.V. Morris · J. Jones

NASA Johnson Space Center, 2101 NASA Parkway, Houston, TX 77058, USA

C. Gundersen

AMU Engineering, Miami, FL 33156, USA

A. Steele

Geophysical Laboratory, Carnegie Institution of Washington, Washington, DC 20015, USA

J. Wray

School of Earth and Atmospheric Sciences, Georgia Institute of Technology, Atlanta, GA 30332, USA

O. Botta

Swiss Space Office, Berne, Switzerland

L.A. Leshin

Department of Earth and Environmental Sciences, Rensselaer Polytechnic Institute, Troy, NY 12180, USA

T. Owen

Institute for Astronomy, University of Hawaii, Honolulu, HI 96822, USA

S. Battel

Battel Engineering, Scottsdale, AZ 85253, USA

B.M. Jakosky

Laboratory for Atmospheric and Space Physics, University of Colorado, Boulder, CO 80303, USA

H. Manning

Concordia College, Moorehead, MN 56562, USA

S. Squyres

Department of Astronomy, Cornell University, Ithaca, NY 14853, USA

R. Navarro-González

Laboratorio de Química de Plasmas y Estudios Planetarios, Instituto de Ciencias Nucleares, Universidad Nacional Autónoma de México, Circuito Exterior, Ciudad Universitaria, Apartado Postal 70-543, México, D.F. 04510, Mexico

C.P. McKay

NASA Ames Research Center, Moffett Field, CA 94035, USA

F. Raulin · R. Sternberg

LISA, Universités Paris Est-Créteil, Paris 7, Denis Diderot et CNRS, CMC, 61 av. du Général de Gaulle, 94010 Créteil cedex, France 


\begin{abstract}
The Sample Analysis at Mars (SAM) investigation of the Mars Science Laboratory (MSL) addresses the chemical and isotopic composition of the atmosphere and volatiles extracted from solid samples. The SAM investigation is designed to contribute substantially to the mission goal of quantitatively assessing the habitability of Mars as an essential step in the search for past or present life on Mars. SAM is a $40 \mathrm{~kg}$ instrument suite located in the interior of MSL's Curiosity rover. The SAM instruments are a quadrupole mass spectrometer, a tunable laser spectrometer, and a 6-column gas chromatograph all coupled through solid and gas processing systems to provide complementary information on the same samples. The SAM suite is able to measure a suite of light isotopes and to analyze volatiles directly from the atmosphere or thermally released from solid samples. In addition to measurements of simple inorganic compounds and noble gases SAM will conduct a sensitive search for organic compounds with either thermal or chemical extraction from sieved samples delivered by the sample processing system on the Curiosity rover's robotic arm.
\end{abstract}

Keywords Mars, organic compounds · Volatiles · Isotopes $\cdot$ Evolved gas analysis $\cdot$ Gas chromatography mass spectrometry $\cdot$ Noble gases $\cdot$ Habitability $\cdot$ Mars Science Laboratory $\cdot$ Curiosity Rover $\cdot$ Gale crater

\title{
1 Introduction
}

The Sample Analysis at Mars (SAM) investigation addresses three primary science questions to contribute to the mission goal of the Mars Science Laboratory (MSL) to quantitatively assess the habitability potential of Mars:

- What does the inventory of carbon compounds, or lack thereof, near the surface of Mars reveal about its potential habitability?

- What are the chemical and isotopic states of the lighter elements in the rocks, soils, and atmosphere and what do these reveal about potential habitability?

- How were past environmental conditions different from today's?

Diverse environments from different epochs of Mars' history have been revealed with increasing detail in recent years through orbital survey and landed missions. This wealth of new data provides new paradigms for understanding the history of Mars (e.g. Bibring et al. 2006a, 2006b, 2006c) as well as unprecedented context and motivation for the geological

A. Buch

Ecole Centrale de Paris, Chatenay-Malabry, France

P. Sorensen · R. Kline-Schoder

Creare Corporation, Hanover, NH 03755, USA

D. Coscia $\cdot$ C. Szopa $\cdot$ S. Teinturier

UPMC Univ. Paris 06, Université Versailles St-Quentin, CNRS/INSU, LATMOS-IPSL, 4 Place Jussieu, 75005 Paris cedex, France

B.P. Block · K. Arnett · R. Miller · C. Edmonson

Space Physics Research Laboratory, University of Michigan, Ann Arbor, MI 48109, USA

S. Gorevan · E. Mumm

Honeybee Robotics Spacecraft Mechanisms Corporation, New York, NY 10001, USA 
and in situ geochemical studies planned for MSL's Curiosity rover after its arrival on the floor of Gale Crater in August of 2012. Recently, Mars Reconnaissance Orbiter has systematically acquired high-resolution imaging and spectroscopic studies of Gale Crater and the surrounding regions (Zurek and Smrekar 2007). These data have enabled a more detailed understanding of the landscape that MSL will traverse and the broader context of this site. MSL's task is to characterize this site with a new generation of robotic analytical tools to identify the most promising rock targets for revealing if conditions on ancient Mars were favorable for life.

The present surface conditions on Mars could be challenging for life given the lack of abundant liquid water, low temperatures, significant surface ultra-violet and near surface cosmic radiation, and potentially oxidizing surface conditions. It is commonly hypothesized that the climate on Mars was more benign several billion years ago, with a thicker atmosphere protected for a time by an early planetary magnetic field (Acuna et al. 1999). Although this hypothesis has been critically examined using climate models (Tian et al. 2010) significant surface water was evidently created, possibly by episodic flooding, subsurface hydrothermal systems, large impacts, and/or atmospheric transport (Carr and Head 2010). At the time when life appears to have originated on Earth (Nisbet and Sleep 2001), it is possible that microbial life may have similarly emerged on Mars drawing from a planetary inventory of elements and carbon-based molecules such as those found in carbonaceous meteorites (e.g. Sephton et al. 2002). If such life existed, it may have been critically dependent on the hypothetical clement conditions of early Mars and unable to adapt to planetary changes. It then might have become extinct or taken refuge below the surface as much as 3.5 billion years ago. The tectonic quiescence of Mars might have enabled preservation of morphological and chemical evidence of biological or pre-biotic activity in sedimentary deposits. Such evidence may still be present in the martian rock record, in contrast to the early organic chemical history of the Earth, which has been substantially altered or erased by perennially active hydrological and tectonic processes. Original organic chemicals in the shallow sediments and exposed rock accessible to a rover, might be destroyed by the significant ionizing radiation and chemical oxidation present at the martian surface. Altered surviving organic compounds may comprise refractory material more resistant to such alteration, with isotopic signatures left as the only clues to its biogenic origin. The SAM investigation together with the other investigations of the powerful MSL scientific payload is designed to explore potential windows into past conditions on Mars.

The ground beneath Curiosity will be probed by the Dynamic Albedo of Neutrons (DAN) instrument as the rover traverses. DAN searches for high subsurface hydrogen abundance associated with hydrated minerals. MSL instruments such as the Mast Camera (Mastcam), the Navigation Cameras (NavCam's), and the Chemistry \& Camera (ChemCam) instruments will help the science team identify targets. The initial study of the targets will employ robotic arm tools: Mars Hand Lens Imager (MAHLI) and the Chemistry and Alpha Particle X-ray Spectrometer (APXS). Subsequently, target samples will be collected and delivered into SAM and the Chemistry and Mineralogy (CheMin) experiment using the Sample Acquisition, Processing, and Handling (SA/SPaH) subsystem. CheMin, located next to SAM in the interior of the rover, is a powder X-ray Diffraction (XRD) instrument that provides mineralogical information. SAM (Fig. 1) complements this experiment, in particular, by analyzing the thermally evolved gas after thermal decomposition of identically manipulated and delivered samples. The SAM evolved gas analysis (EGA) may liberate $\mathrm{SO}_{2}, \mathrm{CO}_{2}$ and $\mathrm{H}_{2} \mathrm{O}$ or other volatiles associated with mineral breakdown. 
Fig. 1 A model of the SAM suite (upper panel) showing the location of the instruments and the sample inlets. Pictures of the suite with and without side panels in place before delivery for integration into the rover are shown in the bottom panes (TLS $=$ Tunable Laser Spectrometer; QMS = Quadrupole Mass Spectrometer; GC = Gas Chromatograph; SSIT $=$ Solid Sample Inlet Tube; SMS = Sample Manipulation System)

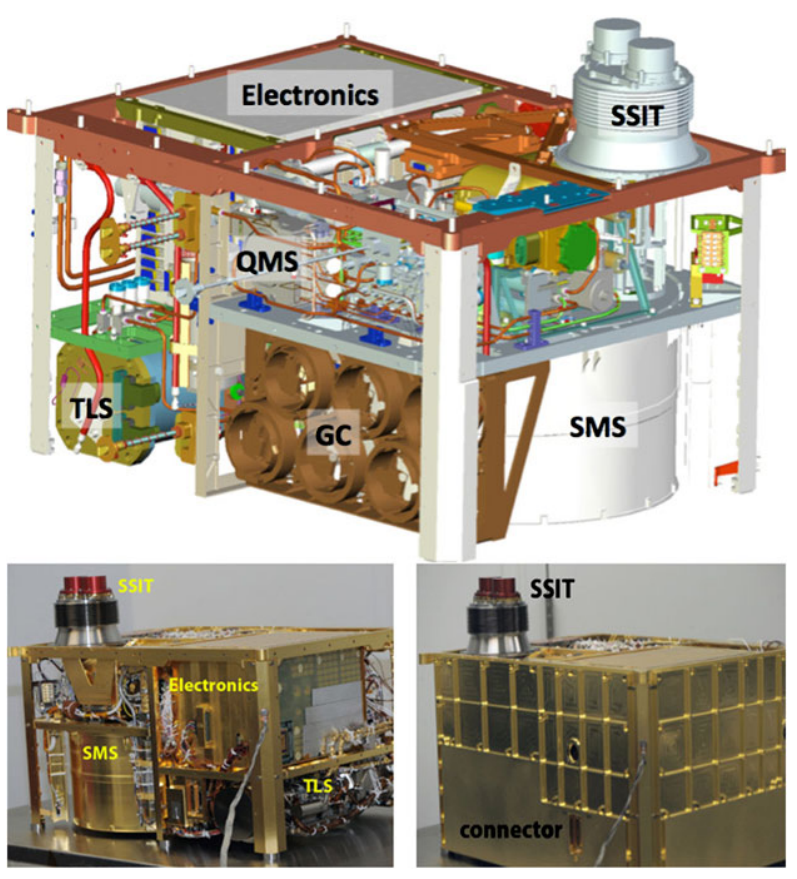

The primary science goals for the MSL mission are to assess the biological potential of the landing site, to characterize its geology and geochemistry, to investigate the role of water, and to measure the spectrum of surface radiation. Investigations of atmospheric composition will provide additional clues to the history of volatiles and climate. This manuscript describes the SAM instrument suite and how the SAM experiments expect to address these mission goals. We summarize the current state of knowledge in several target measurement areas, describe the SAM analytical approach within the MSL operation plan, give an overview of the measurement requirements and capabilities of the individual and integrated SAM instruments (Figs. 1, 2, and 3), and discuss scientific synergies with other MSL investigations. A more detailed description of the SAM suite subsystems (i.e., Quadrupole Mass Spectrometer, QMS; Gas Chromatograph, GC; Tunable Laser Spectrometer, TLS; Gas Processing System, GPS; electronics; SAM calibrants; thermal and mechanical subsystems; and flight software), SAM development and qualification, contamination-control measures, calibration achieved prior to delivery to the Jet Propulsion Laboratory (JPL), and the planned SAM archival products are also presented. Current plans include refinement and testing of new scripts on the SAM testbed (under development) and studies on standards and Mars analogue samples using breadboards that approximate SAM operating conditions. We conclude with a summary of our expectations for the products of the SAM investigation at the Curiosity landing site in Gale Crater.

\section{Overview of SAM Science Goals}

\subsection{Sources and Transformation Paths for Organic Compounds}

A primary goal of the SAM investigation is to carry out a broad and sensitive search for organic compounds and to measure the isotopic composition of carbonaceous material. 


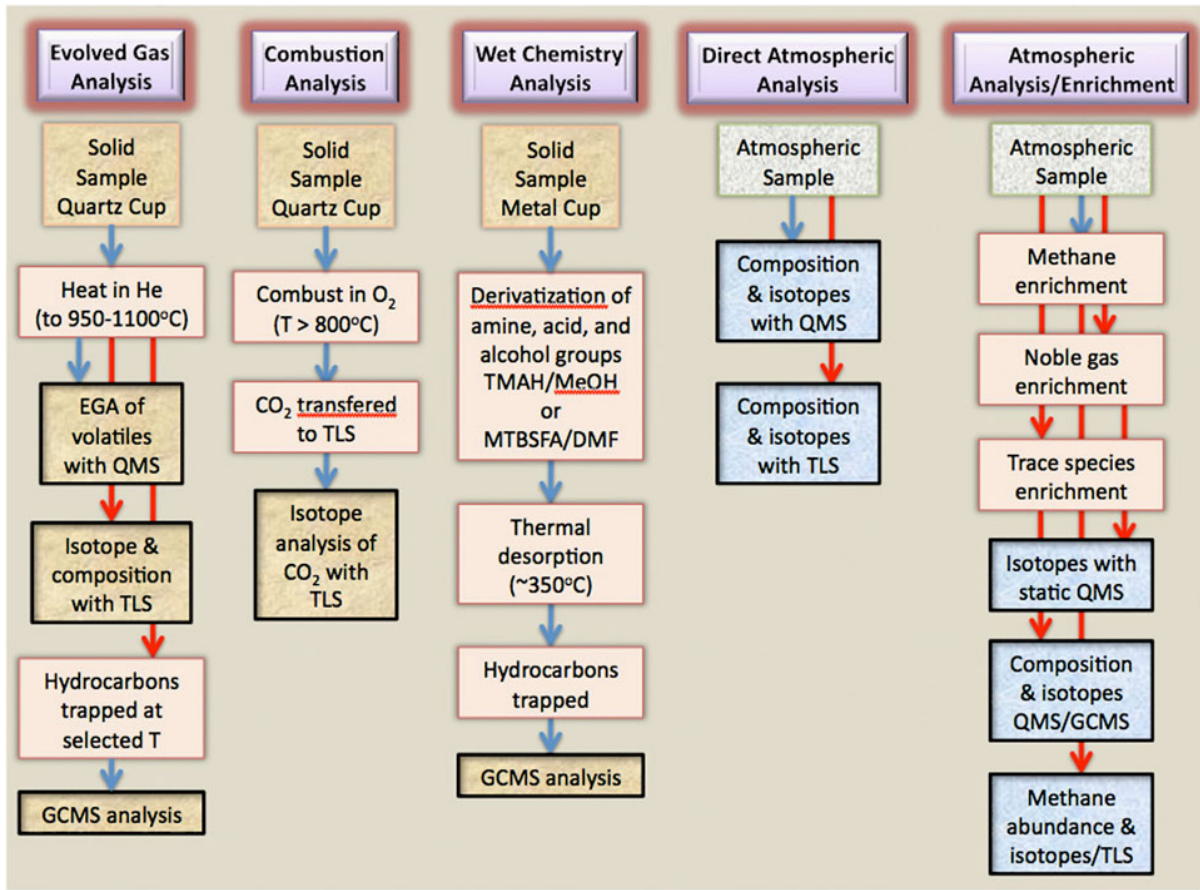

Fig. 2 The three SAM instruments the Quadrupole Mass Spectrometer (QMS), the Gas Chromatograph (GC), and the Tunable Laser Spectrometer (TLS) operate on either atmospheric or solid samples. The SAM inlet funnels and the Sample Manipulation System serve to position and seal a solid sample in an oven for wet or dry gas extraction. Gases produced from solids or introduced directly from the atmosphere can be further processed by a system of chemical scrubbers, traps, and getters that enable a measurement focus on the specific species of interest

Sources of organic compounds that SAM might detect could be exogenous, indigenous, or terrestrial contamination. We will consider exogenously sourced compounds in the following discussion to mean those that are directly derived from in-fall of meteorites, interplanetary dust particles (IDPs), and larger volatile-rich impactors such as comets or carbonaceous asteroids.

Exogenous organic carbon is expected to share chemical characteristics of carbon-rich meteorites. The bulk of the organic carbon in carbonaceous chondrites is in a kerogenlike macromolecular form (e.g. Pizzarello 2006), consisting predominantly of stacked layers of aromatic hydrocarbons linked together by aliphatic carbon chains (Krishnamurthy et al. 1992; Komiya and Shimoyama 1996). However, many pre-biotic compounds, including amino acids, carboxylic acids, and nucleobases, have also been identified in these meteorites (e.g. Botta et al. 2001).

Indigenously sourced compounds could be produced biologically or be geochemical processes on Mars. While no organic compounds have been definitively identified on Mars, there is strong evidence of an indigenous, high-temperature released, reduced organic phase in several martian meteorites (Table 1) based upon data from $\delta^{13} \mathrm{C}$ analysis of Nakhla, and ALH84001 martian meteorites. The source of these meteoritic organics is unclear (e.g. Becker et al. 1997). Indigenous non-biological organic chemistry could come from hydrothermal Fischer-Tropsch type processes by $\mathrm{CO}_{2}$ reduction associated with carbonate deposition, serpentinization reactions, UV catalysis, or hydrothermal 


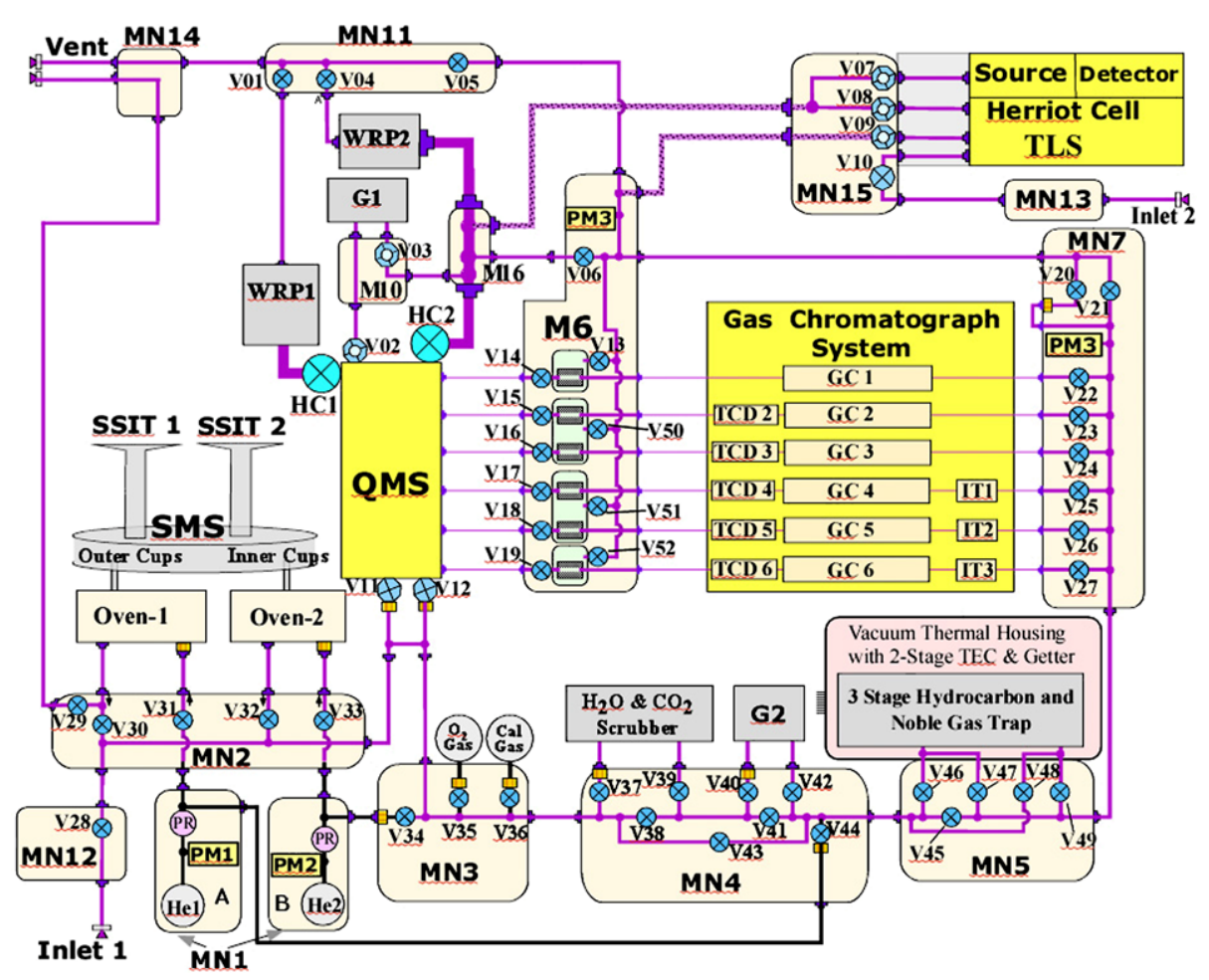

Fig. 3 The SAM detailed gas flow diagram showing the Quadrupole Mass Spectrometer (QMS), the Tunable Laser Spectrometer (TLS), the Gas Chromatograph System, the manifolds (MNx), the microvalves (Vx), the Sample Manipulation System (SMS) with its Solid Sample Inlet Tubes (SSITx), and the High Conductance Valves $(\mathrm{HCx})$. Not shown in this diagram are the manifold and pipe heaters and the temperature sensors associated with each heater

breakdown of siderite (Anpo and Chiba 1992; Zolotov and Shock 2000; McCollom 2003; Steele et al. 2007). In contrast to Earth, where biotic compounds are ubiquitous making it difficult to confirm abiotic geochemical sources, on Mars the ultimate challenges may be detect and confirm biotic sources, should they exist.

\subsection{Organic Compounds of Biotic and Prebiotic Relevance}

If ancient Mars rocks are found to preserve organic compounds, then the priority will be to understand the nature of their source and preservation. On Earth, organisms leave distinctive chemical records in sediments that reflect biological utilization of highly restricted sets of compounds (e.g. amino acids), biosynthesis of ordered patterns in the length and structure of compounds that are, for example, incorporated in microbial cell membranes (Summons et al. 2008) and molecular and isotopic distributions in rock facies that record ecological variance (Eigenbrode 2008). Organic compounds produced by radiation processes in space and then possibly transformed by aqueous processes in the parent body of a carbonaceous meteorite, such as Murchison, demonstrate much different characteristics. In these primitive materials, a wide variety of amino acids are present, most of which are not utilized by terrestrial life. Highly branched alkyl hydrocarbons dominate over the more linear structures common of terrestrial molecular fossils. The odd/even or even/odd enhancements in 
Table 1 Provenance \& carbon isotopes of organics in martian meteorites

\begin{tabular}{|c|c|c|c|}
\hline Meteorite & Type of organic material & $\delta \mathrm{C}^{13}(\%)$ & Lithology \\
\hline \multirow[t]{3}{*}{ ALH84001 } & PAHs & $\mathrm{NM}$ & $\begin{array}{l}\text { Within carbonates } \\
\text { and along silicate } \\
\text { grain boundaries }\end{array}$ \\
\hline & Macromolecular & $\sim-11$ & Ibid \\
\hline & Carbon & -15 & Bulk matrix \\
\hline \multirow[t]{2}{*}{ Nakhla } & Simple aromatics & $\sim-22$ & $\begin{array}{l}\text { Cracks and grain } \\
\text { boundaries }\end{array}$ \\
\hline & Macromolecular carbon & -33 and -15 & Unknown \\
\hline $\begin{array}{l}\text { Los Angeles, QUE94201, } \\
\text { Shergotty, Zagami, DaG 476, } \\
\text { Dho 019, NWA1183, SaU 005, } \\
\text { ALH 77005, LEW 88516, } \\
\text { Y793605, Chassigny }\end{array}$ & Macromolecular carbon & $\sim-20$ & $\begin{array}{l}\text { Silicates (i.e. } \\
\text { pyroxene/olivine) }\end{array}$ \\
\hline
\end{tabular}

Data from (Wright et al. 1992; McKay et al. 1996; Grady et al. 1997, 2004; Jull et al. 1998a, 1998b; Becker et al. 1999; Sephton et al. 2002; Steele et al. 2007)

chain length that are the signatures of terrestrial cellular life are absent. Many of the interesting compounds such as carboxylic acids, nucleobases, and amino acids that might be diagnostic for sources are too polar to be analyzed by pyrolysis-gas chromatography-mass spectrometry (GCMS) alone. For this reason, SAM includes nine cups that contain sealed volumes of liquid chemical compounds designed to produce derivatized products of these polar compounds, making them sufficiently volatile and amenable to SAM GCMS analysis. The measurement protocols using these wet chemistry techniques are described in Sect. 4.6.

\subsection{Chemical and Isotopic States of Inorganic Compounds}

Assessment of potential martian habitability must include an understanding of geochemical context. On Earth this term refers to the many materials and processes that interact with the extensive biosphere, but on Mars the geochemical context is, a priori, an essentially inorganic data set that constrains the search for and interpretation of any organic chemistry there. Such data are primarily acquired by determination of the elemental and mineralogical compositions of rocks and soils; preferably the same samples undergoing organic and isotopic analyses. These data are critical to understanding both the origin and evolution of these samples and the positive or negative results of a survey of their organic content. "First-order" elemental and mineralogical analysis of surface samples will support geological site characterization. This preliminary analysis can support decisions about whether, and from what location, to obtain samples for further analysis by SAM and CheMin. SAM will complement the other payload measurements by generating a set of more detailed and definitive inorganic geochemical analyses than have been possible with previous landed missions. These analyses will provide a geochemical fingerprint of rock type, information of the depositional environment (igneous or sedimentary), and constraints on the nature, history, and extent of aqueous and/or thermal histories of a sample. These martian observations may imply a variety organic formation processes and reveal clues regarding organic preservation potential, as analogous data do on Earth (Summons et al. 2011). The SAM evolved gas analysis will help constrain abundances of the key elements such as $\mathrm{H}, \mathrm{C}, \mathrm{O}$, and $\mathrm{S}$ within the host mineral phases of potential organic compounds. Isotopic measurement on simple evolved gases may 
help interpret data suggestive of life, or lack thereof, and will set the strategy for further analysis, such as repeating the experiment with a longer integration time or analyzing the sample in a subsequent derivatization experiment.

In the chemical associations and spatial distributions of light elements found near the surface of the Earth, the signatures of life are abundant. Our understanding of the dynamic resource exchange between microbial life and its environment (i.e. terrestrial microbial ecology) and the range of environmental characteristics that are conducive to life have advanced greatly since the pioneering Viking search for life on Mars (Toporski and Stale 2004). Tantalizing clues about the former chemical potential of Mars are found in the SNC meteorites (e.g. Bridges and Grady 2000; Bridges and Warren 2006) including the scale and timing of aqueous alteration (e.g. Bouvier et al. 2005). While this information suggests types of environments that might contain life, it is limited by our knowledge of a single suite of organisms having a common origin and evolving in a unique biosphere, Earth. By also looking to Mars, we can dramatically expand our insight into the geochemical and biogeochemical processes that drive light-element chemistries on Earth and its closest neighbor. The key SAM experiment that addresses light-element chemistries is EGA, which will be used to characterize thermal and compositional features of reduced organic components, measure the relative amount of hydration water in minerals, and support the indirect detection of some redox mineral phases having varying degrees of aqueous alteration via thermal profiling of evolved decomposition products. For example, simple gases, such as $\mathrm{CO}_{2}, \mathrm{H}_{2} \mathrm{O}$, and $\mathrm{SO}_{2}$ produced from thermally decomposed carbonates, sulfates, and hydrated minerals, are detected by the QMS and $\mathrm{CO}_{2}$ and $\mathrm{H}_{2} \mathrm{O}$ by the TLS. The QMS and the TLS also measure a suite of isotope ratios in these evolved gases. Light-element chemistry studies of the Gale crater lower mound strata may help reveal variations in ancient environmental conditions and planetary-scale processes on Mars.

\subsection{Atmospheric Composition and Surface Interactions}

The exchange of volatiles between the martian atmosphere and its surface is an important process with respect to habitability potential. Exposed organic matter in the surface sediments is susceptible to redox reactions with atmospheric gases and near-surface sedimentary volatiles, $\mathrm{H}_{2} \mathrm{O}$ in particular, that may be in exchange with the atmosphere. Also, the movement of near-surface volatiles could affect the nature and stability of oxidants with implications for organic degradation. Atmosphere-surface exchange provides information on the nature of the surface as a site for the adsorption of volatiles, which in turn has implications for understanding the climate history of Mars.

The magnitude of the gas exchange between the atmosphere and the surface depends upon the season. In the first 90 sols of the MSL mission there should be measurable changes in the $\mathrm{H}_{2} \mathrm{O}$ and $\mathrm{CO}_{2}$ concentrations in the surface soil due to seasonal effects. Jakosky et al. (1997) argued that measurements from the Viking lander indicate that even diurnal cycles cause more than half of the atmospheric water vapor to exchange with the surface implying that seasonal effects should be even more pronounced. Coordinated measurements (Tamppari et al. 2010) in 2008 from the Phoenix Lander and the Mars Reconnaissance Orbiter allowed both dust and water vapor variability to be studied over part of a seasonal cycle and the extent of the diurnal atmosphere/surface exchange in the polar region to be determined. $\mathrm{CO}_{2}$ will also condense on the surface in the winter latitudes and adsorb into the surface at all latitudes. The magnitude and kinetics of $\mathrm{CO}_{2}$ adsorption will provide important information on the role of the surface in regulating atmospheric pressure. $\mathrm{O}$ and $\mathrm{CO}$, while present in the martian atmosphere at low levels, may also react with surface material in a seasonally 
dependent way due to changes in temperature. SAM will not be expected to measure surface adsorbed $\mathrm{H}_{2} \mathrm{O}$ and $\mathrm{CO}_{2}$ well because of the interval of time between sampling by the MSL arm and delivery into a SAM cup where the sample will be sealed. However it is planned to sample the atmosphere at different points in the diurnal cycle and isotope fractionation will occur as volatiles condense and evaporate. Variations in atmospheric isotopic composition with the precisions provided by the TLS (Sect. 4.2) may provide constraints on the rate or extent of exchange between the atmosphere and surface reservoirs.

\subsection{Atmospheric Evolution Probed through Isotope Measurements of Noble Gases and Light Elements}

Isotopic distributions of the chemically inert noble gases carry no information on past or present biological activity. They are, however, excellent tracers of fractionation due to physical processing. As such, they are powerful recorders of volatile sources on and in terrestrial planets, and markers of processes that could have driven the earliest atmospheres along planet-specific evolutionary paths to the divergent compositional states observed today. The large fractionation in ${ }^{15} \mathrm{~N} /{ }^{14} \mathrm{~N}$ ratio in the martian atmosphere as inferred from Viking mass spectrometer measurements (Nier et al. 1976) has been used as a basis for models (Jakosky et al. 1994) of long term atmospheric loss. Noble gases and nitrogen therefore offer the best chance to address the histories of atmospheric pressure, composition, and a coupled surface-atmosphere volatile exchange system that could have supported development of a native biota on Mars. Progress toward the ultimate objective of understanding all three of the terrestrial planets as a linked class of objects clearly hinges on how much more we can learn about volatile abundance and compositions of noble gases and other atmophile species on Mars and Venus. Section 3.3 expands on specific measurements that are planned for SAM in this regard.

\section{Current Knowledge and Next Steps}

\subsection{Sources and Oxidation Sinks for Organic on Mars}

The fate of organics exogenously delivered to Mars is an intriguing question. Organic molecules have been detected in the interstellar medium (e.g. Ehrenfreund and Charnley 2000; Millar 2004), comets (e.g. Crovisier et al. 2004) as well as meteorites (e.g. Botta and Bada 2002; Sephton 2004). The latter contain on the order of a few percent organic carbon, most of which is locked in an insoluble organic matrix. In addition, a wide variety of compounds, both in the soluble and insoluble fractions, are present in these meteorites, with carboxylic acids being the most abundant distinct class of compounds in carbonaceous chondrites, such as the Murchison meteorite. Organic compounds related to life, including amino acids and nucleobases, have also been detected in a number of carbonaceous chondrites. Most recently, a range of extraterrestrial nucleobases have been identified in carbonaceous chondrites (Callahan et al., 2011), demonstrating again the high potential of abiotic chemical synthesis of organic compounds in a variety of extraterrestrial environments and their delivery to the inner planets in the earliest epochs of solar system formation. Martian meteorites have also been shown to contain some form of reduced carbon (Sect. 2.1 and Table 1).

Considering the bombardment history of planets and satellites in the solar system, including flux rates of different sized particles, and the survival of organic compounds during 
impact as well as their abundances in different objects it is possible to estimate the annual accumulation of organic material on a planetary surface. For Mars, this accumulation has been estimated to be on the order of $10^{6} \mathrm{~kg}$ per year (Flynn 1996), but may have been significantly higher in the past (Chyba and Sagan 1992). The constraints on meteoritic material provided by the MER measurements (Yen et al. 2006) allowed an estimate of hundreds of ppm of meteoritic organic carbon. The Curiosity elemental analysis tools are expected to enable similar constraints on meteoritic contributions to the sampled materials in Gale crater and the sensitivity of SAM for organic carbon is discussed in Sect. 4.2.

The Viking Life Science Experiment package that explored the Martian surface in 1976 detected an extraordinary reactivity of the soil. Specifically, $\mathrm{O}_{2}$ was released upon humidification or wetting the soil in the Viking Gas Exchange (GEx) experiment (Klein et al. 1976; Oyama and Berdahl 1977) and $\mathrm{CO}_{2}$ was produced when an organic solution was added into the soil by the Labeled Release (LR) experiment (Levin and Straat 1976, 1977). Most surprisingly, no organics were detected in the soil when heated up to $500{ }^{\circ} \mathrm{C}$, except for some chlorohydrocarbons that were attributed to terrestrial contamination from cleaning fluids in the gas chromatograph mass spectrometer (GCMS) experiments (Biemann et al. 1976, 1977). Research following the Viking mission focused on laboratory simulations under martian surface conditions aimed to identify the underlying chemical mechanisms that may explain the Viking results. Interpretations suggest the presence of different types of oxidants in the Martian soil: (1) a thermally labile oxidant at levels $>1$ ppm for the LR experiment; (2) thermally stable oxidants at levels of $10 \mathrm{ppm}$ for the GEx experiment and at $\leq 0.1 \%$ for explaining the lack of organics by the GC-MS experiments (Navarro-Gonzalez et al. 2010) and (3) a weak oxidant (e.g., Fe(III)) required to explain the slow oxidation of nutrient(s) in the LR experiment (Zent and McKay 1994) and the lack of organics by the GC-MS experiments. Table 2 lists different types of oxidants that have been suggested in the literature to explain the Viking results. Of these, the only oxidants detected, to date, are $\mathrm{H}_{2} \mathrm{O}_{2}$ in the Martian atmosphere (Clancy et al. 2004; Encrenaz et al. 2004), Fe(III) in Fe-bearing phases such as nanophase iron-oxides and jarosite (e.g. Klingelhofer et al. 2004), and perchlorate (Hecht et al. 2009) in the Martian soil. It has been suggested that potentially large quantities of $\mathrm{H}_{2} \mathrm{O}_{2}$ in the soil may result from geologic processes (Hurowitz et al. 2005) or electrochemistry triggered by triboelectric process in the Martian convective storms (Atreya et al. 2006; Delory et al. 2006). A recent 1D photochemical model (Zahnle et al. 2008) postulates a major surface sink for reactive oxygen in the form of $\mathrm{O}_{3}$ and $\mathrm{H}_{2} \mathrm{O}_{2}$ for both the present atmosphere and postulated earlier atmospheres with different solar forcing and $\mathrm{CO}_{2}$ and $\mathrm{H}_{2} \mathrm{O}$ abundances.

Using a Wet Chemistry Laboratory instrument the 2008 Phoenix lander mission detected perchlorates in a sample of Martian soil from the polar region (Hecht et al. 2009). If these polar perchlorates were produced by gas phase chemistry in the atmosphere following volcanic activity (Catling et al. 2010) they could be widely distributed around the planet. With regard to their impact on an EGA analysis of a martian sample, perchlorates can promote the combustion of organic compounds under high temperatures. During pyrolytic processes in the experiment ovens Phoenix analyzed five soil samples with the Thermal and Evolved Gas Analyzer (TEGA) instrument, using thermal gas evolution, and did not report any organic material or its decomposition products within a mass range of 2-140 Dalton. In one TEGA experiment, however, a mid-temperature $\left(400-600^{\circ} \mathrm{C}\right)$ release of $\mathrm{CO}_{2}$ was speculated to be the result of combustion of organics by soil oxidants although other possible sources of this $\mathrm{CO}_{2}$ release include $\mathrm{Mg}$ or $\mathrm{Fe}$-carbonate, or $\mathrm{CO}_{2}$ adsorbed to mineral grains (Boynton et al. 2009). The presence of perchlorates and other oxidants, if present in the martian soil and rocks at Gale crater, could have implications for the SAM search for organics as some of 
Table 2 Oxidants suggested to explain the Viking results ${ }^{\mathrm{a}}$

\begin{tabular}{|c|c|}
\hline Oxidant & Notes and reference \\
\hline \multicolumn{2}{|c|}{ Gas exchange experiment release of $\mathrm{O}_{2}$ on humidification or wetting } \\
\hline $\mathrm{KO}_{2}$ & Ponnamperuma et al. (1977) \\
\hline $\mathrm{ZnO}_{2}$ & Ponnamperuma et al. (1977) \\
\hline $\mathrm{Ca}\left(\mathrm{O}_{2}\right)_{2}$ & Ponnamperuma et al. (1977) \\
\hline $\mathrm{MnO}_{2}$ & Irradiated (Blackburn et al. 1979) \\
\hline $\mathrm{H}_{2} \mathrm{O}_{2}$ & Huguenin et al. (1979) \\
\hline Oxyhalides & $\begin{array}{l}\text { Of chlorine and bromine }\left(\mathrm{ClO}_{4}^{-}, \mathrm{ClO}_{3}^{-}, \mathrm{ClO}_{2}^{-}, \mathrm{ClO}^{-}, \mathrm{BrO}_{4}^{-}, \mathrm{BrO}_{3}^{-}, \mathrm{BrO}_{2}^{-}, \mathrm{BrO}^{-} \text {) }\right. \\
\text { (Zent and McKay 1994) }\end{array}$ \\
\hline Oxyhalides & of chlorine $\left(\mathrm{ClO}_{4}^{-}, \mathrm{ClO}_{3}^{-}, \mathrm{ClO}_{2}^{-}, \mathrm{ClO}^{-}\right)($Quinn et al. 2011) \\
\hline \multicolumn{2}{|c|}{ Labeled release of added nutrient } \\
\hline $\mathrm{H}_{2} \mathrm{O}_{2}$ & Ponnamperuma et al. (1977); Levin and Straat (1981) \\
\hline $\mathrm{NOO}_{2}^{-}$ & Peroxonitrite (Plumb et al. 1989) \\
\hline Clays & Fe-rich smectite clays (Banin and Margulies 1983) \\
\hline Oxyhalides & of chlorine $\left(\mathrm{ClO}_{4}^{-}, \mathrm{ClO}_{3}^{-}, \mathrm{ClO}_{2}^{-}, \mathrm{ClO}^{-}\right)($Quinn et al. 2011) \\
\hline \multicolumn{2}{|c|}{ Lack of organics by GCMS in soil } \\
\hline Superoxide ions & $\begin{array}{l}\text { (Yen et al. 2000) laboratory studies of plagioclase feldspar exposed to UV and } \\
\text { oxygen }\end{array}$ \\
\hline Fe-rich soils & (Nano-phase iron oxide in palagonite or jarosite) (Navarro-Gonzalez et al. 2006) \\
\hline $\mathrm{ClO}_{4}^{-}$ & Perchlorate (Navarro-Gonzalez et al. 2010) \\
\hline
\end{tabular}

adapted from Zent and McKay (1994)

these compounds could be transformed to $\mathrm{CO}_{2}$ in the oven (Navarro-Gonzalez et al. 2009, 2010). If perchlorates are present, a small amount of the organics may form chlorohydrocarbons that could be detected by the SAM GCMS experiment. The presence of perchlorates may also be indicated by $\mathrm{O}_{2}, \mathrm{HCl}$, or $\mathrm{Cl}_{2}$ evolution in a SAM EGA experiment or these compounds may be detected by the ChemCam instrument (Schroder et al. 2011). Similarly, the presence of high concentration $\mathrm{H}_{2} \mathrm{O}_{2}$ in sediments could manifest itself in the form of formaldehyde or methanol, species that are potentially detectable by SAM.

An alternative explanation (Benner et al. 2000) for the lack of evidence for organic compounds in soil analyzed by the Viking GCMS suggested that all organic molecules that would be delivered to the Martian surface by meteorites and comets would most likely be converted to carboxylic acid derivatives that are non-volatile and therefore not easily detected directly by that GCMS.

Ancient indigenous organic molecules could be also destroyed or transformed by the ionizing radiation in the shallow subsurface of Mars. Due to a thin martian atmosphere and lack of magnetic field, the surface of Mars has been bombarded continuously by the energetic particles of the galactic and solar cosmic rays (GCRs and SCRs) for much of its history. Unlike UV radiation which is absorbed in the first mm of soil (Mancinelli and Klovstad 2000; 
Cockell et al. 2005), GCRs can penetrate down to $\sim 1$ meter below the surface (Dartnell et al. 2007). Over the long period of exposure, cosmic rays particles have the capacity to transform complex organic compounds into macromolecules having different, more refractory chemistry and/or into smaller molecules broken from a parent molecule. The latter case may occur either by direct impacts or by secondary reaction with oxidative radicals produced by radiation in the immediate vicinity of the organic molecules (Dartnell et al. 2008). It is not clear how such long-term degradation would affect SAM's measurements of organic compounds at the ancient geologic outcrops because the rates of erosion are highly variable on Mars (Golombek et al. 2006). Erosion of the ancient rock would naturally expose "fresh" (less irradiated) material to the surface with potentially "unbroken" organic molecules. Furthermore, SCRs, which are less energetic than GCRs, cannot penetrate and destroy organic matter deeper than $2 \mathrm{~cm}$ below the surface (Pavlov 2011). Therefore, MSL's drilling and sampling of outcrops from $5 \mathrm{~cm}$ below the surface will exclude the effects of degradation of organic matter by solar cosmic rays. Finally, using the radiolysis constants of amino acids Kminek and Bada (2006) and Pavlov (2011) demonstrated that simple organic compounds with masses below $100 \mathrm{amu}$, should have a good chance to survive long-term exposure to GCRs in the shallow subsurface even extremely low surface erosion rates. Results from Curiosity's Radiation Assessment Detector (RAD) will provide modern radiation characteristics that will help improve long-term modeling of the surface radiation on Mars and possibly constrain its affects on near surface organic chemistry.

\subsection{Atmospheric Composition}

The terrestrial planets have all evolved differently. Mars has taken a path that resulted in a very thin atmosphere and cold surface temperatures with no firm evidence of surface liquid water. On Earth, $\mathrm{CO}_{2}$ is largely sequestered in sedimentary rocks, while moderately warm temperatures $(288 \mathrm{~K})$ still allow vast reservoirs of liquid water to exist on the surface. Venus is believed to have undergone an episode of runaway greenhouse warming early in its geologic history that resulted in a high surface temperature of $730 \mathrm{~K}$, the loss of nearly all of its water, and retention of most of the planet's $\mathrm{CO}_{2}$ in the atmosphere.

With an average surface temperature of $220 \mathrm{~K}$ on Mars today, water presently exists as ice in the polar caps and elsewhere largely in the subsurface although possible minor mid-latitude flows in the equatorial summer have recently been reported (McEwen et al. 2011). However, Mars may harbor much greater quantities of water than those in these ice reservoirs. The $\mathrm{D} / \mathrm{H}$ ratio of $\mathrm{H}_{2} \mathrm{O}$ in the martian atmosphere suggests that a significant fraction of the water that can exchange with the atmosphere has been lost to space (Leshin 2000) but perhaps liquid water still exists in deep subsurface aquifers. There certainly is an abundance of water in near surface hydrated minerals as demonstrated by the neutron detector instrument (Mitrofanov et al. 2004) on the Mars Odyssey spacecraft, the infrared spectroscopy measurements from Mars Express and MRO (e.g. Murchie et al. 2009), and numerous discoveries from the MER mission rovers (e.g. Ming et al. 2008).

Uncertainties in the magnitude of the volcanic outgassing sources and sinks (atmospheric escape to space or to the surface) of atmospheric $\mathrm{CO}_{2}$ result in a corresponding uncertainty in the age of the present atmosphere (Gillmann et al. 2009). While a vigorous outgassing predicts a young $(\sim 1 \mathrm{Ga})$ current $\mathrm{CO}_{2}$ atmosphere (Gillmann et al. 2009) production of the atmosphere from a reduced mantle (Hirschmann and Withers 2008) may have limited $\mathrm{CO}_{2}$ degassing. Carbonates, which store much of the Earth's $\mathrm{CO}_{2}$, have been detected at only low abundance regionally on Mars, such as the Nili Fossae region from orbital observations (Ehlmann et al. 2008) and in situ by the Spirit Rover (Morris et al. 2010). Nevertheless, a recent analysis of the Phoenix mass spectrometer data (Niles et al. 2010) found 
Table 3 Martian lower atmospheric composition measurements

\begin{tabular}{|c|c|c|}
\hline Species & Mixing ratio & Notes \\
\hline $\mathrm{CO}_{2}$ & $\sim 0.95$ & \multirow{8}{*}{$\begin{array}{l}\text { Viking mass spectrometer atmosphere measurements at the Mars } \\
\text { surface }(\text { Owen et al. 1977). Mixing ratio changes with season and } \\
\text { latitude as } \mathrm{CO}_{2} \text { deposits on the polar caps. Ozone mixing ratio is } \\
\text { highly variable (Fast et al. 2006a, 2006b; Perrier et al. 2006) and } \\
\text { anti-correlated with water mixing ratio }\end{array}$} \\
\hline $\mathrm{N}_{2}$ & $2.7( \pm 0.5) \times 10^{-2}$ & \\
\hline${ }^{40} \mathrm{Ar}$ & $1.6( \pm 0.3) \times 10^{-2}$ & \\
\hline $\mathrm{O}_{2}$ & $1.3( \pm 0.3) \times 10^{-3}$ & \\
\hline $\mathrm{Ne}$ & $2.5(+3.5 /-1.5) \times 10^{-3}$ & \\
\hline $\mathrm{Kr}$ & $\sim 300 \mathrm{ppb}$ & \\
\hline $\mathrm{Xe}$ & $\sim 80 \mathrm{ppb}$ & \\
\hline $\mathrm{O}_{3}$ & $0.04-0.2 \mathrm{ppm}$ (variable) & \\
\hline $\mathrm{H}_{2} \mathrm{O}$ & $\sim 2 \times 10^{-4}$ (variable) & $\begin{array}{l}\text { Global average. Highly variable with season and latitude (Smith } \\
\text { 2004; Maltagliati et al. 2011) }\end{array}$ \\
\hline $\mathrm{CO}$ & $\sim 1 \times 10^{-3}$ & From microwave spectroscopy (Clancy et al. 1983) \\
\hline $\mathrm{CH}_{4}$ & $10( \pm 5) \mathrm{ppb}$ & $\begin{array}{l}\text { From Mars Express (Formisano et al. 2004) or ground based high } \\
\text { resolution infrared spectroscopy (Krasnopolsky et al. 2004; } \\
\text { Mumma et al. 2009) }\end{array}$ \\
\hline $\mathrm{H}_{2} \mathrm{O}_{2}$ & $10-800 \mathrm{ppb}$ & Ground based infrared spectroscopy (Encrenaz et al. 2004) \\
\hline
\end{tabular}

Table 4 Global upper limits to possible trace species in the Martian atmosphere

\begin{tabular}{|c|c|c|}
\hline Species & Mixing ratio & Notes \\
\hline $\mathrm{C}_{2} \mathrm{H}_{2}$ & $2 \times 10^{-9}$ & Derived from Mariner 9 infrared data (Maguire 1977). Additional species and their \\
\hline $\mathrm{C}_{2} \mathrm{H}_{4}$ & $5 \times 10^{-7}$ & upper limits from this investigation are $\mathrm{H}_{2} \mathrm{~S}\left(1 \times 10^{-7}\right)$; OCS $\left(1 \times 10^{-8}\right)$; $\mathrm{PH}_{3}$ \\
\hline $\mathrm{C}_{2} \mathrm{H}_{6}$ & $4 \times 10^{-7}$ & $\left(1 \times 10^{-7}\right) ; \mathrm{NH}_{3}\left(5 \times 10^{-9}\right) ; \mathrm{NO}_{2}\left(1 \times 10^{-8}\right) ; \mathrm{N}_{2} \mathrm{O}\left(1 \times 10^{-7}\right)$ \\
\hline $\mathrm{CH}_{2} \mathrm{O}$ & $3 \times 10^{-9}$ & High resolution ground based spectroscopy (Krasnopolsky et al. 1997) \\
\hline $\mathrm{SO}_{2}$ & $3 \times 10^{-10}$ & Ground based infrared spectroscopy (Encrenaz et al. 2011a, 2011b) \\
\hline
\end{tabular}

a $\delta^{13} \mathrm{C}_{\mathrm{VPDB}}=-2.5 \pm 4.3$ per mil that was interpreted as an atmospheric contribution from ongoing volcanic outgassing and carbonate formation. Impact erosion is yet another process that could remove an early massive atmosphere (Melosh and Vickery 1989, Owen and Barnun 1995a, 1995b). Perhaps a massive loss of $\mathrm{CO}_{2}$ atmosphere and/or burial of early carbonate deposits by impact ejecta and volcanism (Michalski and Niles 2010) are responsible for the lack of evident carbonates on the surface of Mars. However, recent analog studies suggest that iron oxide rock coatings and clay-carbonate mixtures obscure NIR and mid-IR carbonate spectral signatures, which may account somewhat for their lack of detection from orbit (Bishop et al. 2011). SAM should secure a $\delta^{13} \mathrm{C}$ with improved precision from past measurements (Sect. 4.2) and together with CheMin continue the search for carbonates in Gale crater.

Much of our knowledge concerning Mars' atmospheric composition was derived from the Mariner 9 and Viking missions, studies of martian meteorites, and additional valuable data on water vapor variability and distribution from more recent missions including Mars Odyssey, Mars Global Surveyor and Mars Reconnaissance Orbiter. The presently known composition of the martian atmosphere is summarized in Table 3. Several constituents for which only upper limits have been determined and are listed in Table 4. A brief summary of chemical pathways leading up to key trace constituents in the atmosphere is given below. 
From Viking measurements (Owen et al. 1977, 1992), the principal constituents of the martian atmosphere are $\mathrm{CO}_{2}, \mathrm{~N}_{2}$ and radiogenic argon, ${ }^{40} \mathrm{Ar}$, in roughly $95 \%, 2.7 \%$ and $1.6 \%$ proportion by volume respectively. $\mathrm{CO}$ and $\mathrm{O}_{2}$ are photolysis products of $\mathrm{CO}_{2}$. The ratio $\mathrm{CO} / \mathrm{O}_{2}$ is found to be 0.54 , rather than 2 , which is expected from their chemical production mechanism:

$$
\begin{aligned}
& \mathrm{CO}_{2}+h v \rightarrow \mathrm{CO}+\mathrm{O}, \quad \text { followed by } \\
& \mathrm{O}+\mathrm{O}+\mathrm{M} \rightarrow \mathrm{O}_{2}+\mathrm{M}, \quad \text { where } \mathrm{M}=\mathrm{CO}_{2} .
\end{aligned}
$$

This is because the abundances of $\mathrm{CO}$ and $\mathrm{O}_{2}$ are regulated by water vapor in the atmosphere of Mars to maintain the escape fluxes of hydrogen and oxygen in the 2:1 ratio- their stoichiometric ratio in $\mathrm{H}_{2} \mathrm{O}$ (McElroy 1972).

Ozone results from the reaction between oxygen atoms and $\mathrm{O}_{2}$ molecules in the atmosphere of Mars in the same manner as on Earth. The ozone abundance on Mars varies seasonally by up to a factor of 100 and is anticorrelated with the seasonal behavior of water vapor due to the loss of $\mathrm{O}_{3}$ on $\mathrm{OH}$, a photolysis product of $\mathrm{H}_{2} \mathrm{O} . \mathrm{H}_{2}$ was detected in the upper atmosphere (Krasnopolsky and Feldman 2001) and is ultimately derived from $\mathrm{H}_{2} \mathrm{O}$ following the recombination of $\mathrm{H}$ atoms, another product of the $\mathrm{H}_{2} \mathrm{O}$ photolysis. Hydrogen peroxide $\left(\mathrm{H}_{2} \mathrm{O}_{2}\right)$ is the only trace constituent detected so far that shows the coupling between the oxygen and hydrogen chemistry of the atmosphere. Long suspected to be present on Mars and predicted by photochemical models (Krasnopolsky 1993; Atreya and $\mathrm{Gu}$ 1994), $\mathrm{H}_{2} \mathrm{O}_{2}$ was finally detected in 2003 (Encrenaz et al. 2003; Clancy et al. 2004; Encrenaz et al. 2004). The trace levels predicted in the atmosphere combined with the $\mathrm{SAM} / \mathrm{QMS}$ background signal in this region of the mass spectra may make an in situ measurement of this species difficult or impossible. On the other hand, if large quantities of hydrogen peroxide are present in the soil, SAM/QMS could potentially infer its presence indirectly by detection of methanol, formaldehyde or other products of surface mineral reactions (Sect. 3.1).

The detection of trace quantities of methane (Table 3) with the Planetary Fourier Spectrometer on Mars Express was reported in 2004 (Formisano et al. 2004; Geminale et al. 2011). Ground-based disk-average observations with the Fourier Transform Spectrometer on the Canada France Hawaii Telescope were also reported that year (Krasnopolsky et al. 2004). A global methane abundance of approximately 10 ppbv was obtained from both sets of data. The Mars Express data also indicated a non-uniform distribution of methane over Mars, with abundance three times greater than average in certain regions. From groundbased observations made between 2003 and 2006 with the Cryogenic Echelle Spectrograph (CSHELL) at the NASA Infrared Telescope Facility (IRTF) and the Near Infrared Echelle Spectrograph (NIRSPEC) on the Keck II telescope, Mumma et al. (2009) reported that, when present, methane was found in large quantities in localized plumes. However, Zahnle et al. (2011) have argued that the methane of these data may not be of martian origin, since the doppler-shifted ${ }^{12} \mathrm{CH}_{4}$ feature attributed to Mars overlaps with the ${ }^{13} \mathrm{CH}_{4}$ isotope of the Earth's atmosphere, which is twenty times stronger. While there is currently no clear resolution for this problem, the data could still be consistent with a small amount of methane of a few ppbv to $10 \mathrm{ppbv}$ at Mars. The variability of methane at Mars is less convincing, however, since none of the present measurement techniques is optimal for the detection of a weak signature of methane. Nevertheless, if confirmed, methane is a key trace constituent for Mars habitability. Its origin whether attributed to either geology or biology, now or in the past requires liquid water (e.g. Atreya et al. 2007). If methane is being produced today, it implies the presence of liquid water in the martian subsurface. Considering the potential 
implications of methane to the habitability or geology of Mars, the Tunable Laser Spectrometer of the SAM Suite is designed to detect methane above $0.3 \mathrm{ppbv}$ and to measure the ${ }^{12} \mathrm{C} /{ }^{13} \mathrm{C}$ isotope with a precision of $\pm 2 \%$ (Webster and Mahaffy 2011).

The molecules listed in Table 4 have not yet been detected, but some are noteworthy. Photochemistry of any methane in the martian atmosphere would result in formaldehyde $\left(\mathrm{CH}_{2} \mathrm{O}\right)$, methanol $\left(\mathrm{CH}_{3} \mathrm{OH}\right)$ and hydrocarbons such as ethane and propane $\left(\mathrm{C}_{2} \mathrm{H}_{6}, \mathrm{C}_{3} \mathrm{H}_{8}\right)$. Models have suggested (Wong et al. 2003) that photochemistry would produce only parts per trillion levels of these constituents even with 100 ppmv of methane present in the atmosphere. On the other hand, the quantities of ethane and propane could be much greater in the immediate vicinity of active methane vents, or if methane is produced presently by a geological process. Serpentinization, followed by metal-catalyzed Fisher-Tropsh reactions, produces heavy hydrocarbons as well as $\mathrm{CH}_{4}$ (McCollom and Simoneit 1999; Sherwood Lollar et al. 2002). Similarly, although the global upper limits of the sulfur and halogen species in Table 4 are low, the local variability can effectively be studied with the MSL instruments. The atmospheric enrichment experiments to be validated on the SAM testbed are expected to solidify detection thresholds for a number of the trace constituents listed in Tables 3 and 4.

\subsection{Isotopic Composition of Atmospheric and Evolved Gas}

Studies of the source and evolution of the martian atmosphere depend on high precision measurements of abundances and isotope ratios of atmospheric gases. SAM is equipped to provide an improvement in the accuracy of existing values measured in situ by 10 to 100 times (Mahaffy 2009; Webster and Mahaffy 2011). Even more interesting will be the direct comparison that can be made with the $\mathrm{C}, \mathrm{O}$, and $\mathrm{H}$ isotopic composition in $\mathrm{CO}_{2}$ and $\mathrm{H}_{2} \mathrm{O}$ in the present atmosphere with the isotopic values from these same gases evolved from ancient rocks in Gale crater. These data are expected to further constrain models of atmospheric loss and provided additional insight into formation and processing paths for these minerals.

Since the noble gas in situ measurements by the Viking GCMS (Owen et al. 1977) there have been several laboratory investigations of the gases trapped in martian meteorites (Bogard et al. 2001). The most straightforward measurement of noble gases is the grand ratio ${ }^{36} \mathrm{Ar} /{ }^{84} \mathrm{Kr} /{ }^{130} \mathrm{Xe}$. This ratio appears remarkably similar on Mars and Earth, suggesting the possibility of a common outside source. Comets have been suggested as a possibility, since the ${ }^{84} \mathrm{Kr} /{ }^{130} \mathrm{Xe}$ ratio in chondritic meteorites is 10 to 20 times smaller than the value in the atmospheres of Earth and Mars (Owen et al. 1992). The Mars-Earth similarity also applies to the relative abundances of the isotopes of Xe (Owen et al. 2009). SAM will evaluate the extent of these two similarities, and the Rosetta Mission in 2014 is expected to provide complementary measurements addressing the possible role of comets in delivering noble gases to the terrestrial planets (Schwehm and Hechler 1994).

The combination of Viking in situ measurements and laboratory analyses of gases trapped in martian meteorites has provided the foundation for a quantitative view of the present state and possible evolutionary history of martian volatiles. However, these two data sets are not in complete concordance, raising concerns about possible unidentified systematic errors in the Viking data or contamination or alteration of the gases captured by the meteorites. The improved in situ accuracy expected for SAM measurements will further constrain current modeling of atmospheric origin and evolution. For example, martian meteorite analyses of the crucial ${ }^{36} \mathrm{Ar} /{ }^{38} \mathrm{Ar}$ ratio have yielded values ranging from 4.1 to as low as 3.4 (Garrison and Bogard 1998), while Viking measurements detected no deviation from the terrestrial value of 5.3. New, more accurate in situ analyses by SAM will provide 
data to reconcile the differences in this ratio. Early in the landed mission SAM is planned to measure precise values for a range of isotope ratios in $\mathrm{CO}_{2}, \mathrm{H}_{2} \mathrm{O}$, and $\mathrm{CH}_{4}$. These ratios secured from atmospheric gases can then be compared with volatiles in the minerals contained in the layers of Gale crater. This comparison combined with the better understanding of atmospheric loss rates from the present atmosphere derived from MAVEN mission measurements will provide further constraints on models of atmospheric loss. In particular, the analysis of $\mathrm{C}$ and $\mathrm{O}$ isotope ratios in ancient rocks will constrain models of contributions to atmospheric evolution from outgassing, hydrodynamic escape, sputtering, and photochemical escape as these make specific predictions regarding changes in the isotopic composition over time (Pepin 1994).

The ${ }^{36} \mathrm{Ar} /{ }^{38} \mathrm{Ar}$ ratio is important because it can constrain models of martian atmospheric escape that will decrease this ratio from its solar value of 5.5 and outgassing from the planet's interior (Pepin 1991) that should drive this ratio in the direction of the primordial value. Viking found the ${ }^{36} \mathrm{Ar} /{ }^{38} \mathrm{Ar}$ of the near-surface martian atmosphere to be $5.5 \pm 1.5$, equal within measurement uncertainty to the solar value. In martian meteorites this ratio ranges from an average of $~ 4.0$ for atmospheric gas trapped in shock-glass (Bogard 1997) to over 5.26 for high-temperature extracts from Chassigny, believed to represent a martian mantle component (Mathew and Marti 2001). There are similar inconsistencies in the Viking determined ${ }^{40} \mathrm{Ar} /{ }^{36} \mathrm{Ar}$ ratio reported as 3000 (Owen et al. 1977) and analyses of shock-glass from martian meteorites where Zagami and EET 79001 showed ${ }^{40} \mathrm{Ar} /{ }^{36} \mathrm{Ar}$ of 1500 to 2177 (Bogard 1997). Precise measurements of Mars' current atmospheric argon composition by SAM are enabled by its static sampling mode.

The isotopic composition of martian neon was not determined by Viking, but was assumed to have ${ }^{20} \mathrm{Ne} /{ }^{22} \mathrm{Ne}$ of $10 \pm 3$ in estimates of elemental abundance from Viking observations (Owen et al. 1977). Measurement of neon trapped in martian meteorites is complicated by inclusion of cosmogenic and terrestrial components, but recent analysis of shergottites suggests that two distinct neon components of martian origin are present, presumably derived from the atmosphere and interior of the planet (Mohapatra et al. 2009). SAM measurements of the martian atmospheric neon composition will help constrain mixing models devised to interpret the meteoritic trapped gases.

The ratio ${ }^{15} \mathrm{~N} /{ }^{14} \mathrm{~N}$ measured by Viking yielded $\delta^{15} \mathrm{~N}$ of $\sim 620 \%$ compared to the terrestrial standard (Owen et al. 1977). This enrichment was explained (McElroy et al. 1976) as a consequence of the preferential escape of ${ }^{14} \mathrm{~N}$ resulting from predissociation of $\mathrm{N}_{2}$ and then further examined by additional models (Fox and Dalgarno 1980; Fox 1993). The D/H ratio of the martian atmosphere is also highly mass fractionated compared to the Earth's oceans, with ground-based spectra revealing $\delta \mathrm{D}$ of $\sim 4500$, about 4 times the terrestrial value (Owen et al. 1988). However, martian meteorites appear to contain a mixture of an atmospheric component and a mantle-derived component with $\delta \mathrm{D} \sim 900 \%$ (e.g. Watson et al. 1994; Leshin 2000). More precision in the ${ }^{15} \mathrm{~N} /{ }^{14} \mathrm{~N}$ and $\mathrm{D} / \mathrm{H}$ measurements by SAM will allow an improved estimate of past nitrogen and hydrogen escape from the planet, a fundamental component of atmospheric evolution models. The atmospheric ${ }^{13} \mathrm{C} /{ }^{12} \mathrm{C}$ and ${ }^{18} \mathrm{O} /{ }^{17} \mathrm{O} /{ }^{16} \mathrm{O}$ ratios also reflect a competition between loss processes to space that make the atmospheric gases heavier and loss processes to the surface such as carbonate formation that can work in the opposite direction. For example, a recent hydrodynamic thermosphere-ionosphere model (Tian et al. 2009) predicts early Noachian massive $\mathrm{CO}_{2}$ loss resulting from the strong EUV flux present at that time giving rise to atmospheric conditions that would not support surface liquid water. Only later in the mid and late Noachian were the EUV conditions sufficient for enough of a $\mathrm{CO}_{2}$ atmosphere to develop that surface liquid water could be sustained. The authors suggest that observed $\mathrm{C}$ and $\mathrm{O}$ isotope fractionation patterns could be used to test this hypothesis. The TLS $\mathrm{CO}_{2}$ isotope measurements are intended to provide this data. 
The TLS component of SAM will have greater sensitivity than ground-based techniques for atmospheric methane detection, which will address the present controversy arising from remote spectral observations (Zahnle et al. 2011). In addition to abundance measurements, SAM will be able to determine the ${ }^{13} \mathrm{C} /{ }^{12} \mathrm{C}$ ratio of methane with high accuracy if it is present in the atmosphere at the $10 \mathrm{ppb}$ level (Webster and Mahaffy 2011). Measurements of the ${ }^{13} \mathrm{C} /{ }^{12} \mathrm{C}$ ratio in organic macromolecular material in martian meteorites (Jull et al. 1998a, 1998b; Grady et al. 2004) overlap with ranges common of terrestrial biogenic carbon and certainly no single carbon isotope signature can establish biogenicity. Nevertheless, the SAM TLS measurements of carbon isotopes from the multiple sources of atmospheric methane, atmospheric carbon dioxide, combusted organics, and methane and carbon dioxide evolved from solid samples would greatly advance our understanding of the carbon reservoirs of Mars.

The Phoenix mass spectrometer secured both $\mathrm{C}$ and $\mathrm{O}$ isotope measurements in $\mathrm{CO}_{2}$. This in situ oxygen measurement of $\delta^{18} \mathrm{O}$ of $31.0 \pm 5.7$ per mil (VSMOW) was a substantial improvement in precision over the Viking value of $0.0 \pm 50 \%$ (Nier and Mcelroy 1977) and recent ground-based spectroscopic observations (Krasnopolsky et al. 2007). Martian meteorite analysis has shown that the oxygen isotopic composition of carbonates and hydroxylbearing minerals are displaced from the principal fractionation line defined by silicates, implying the existence of at least two distinct volatile reservoirs on Mars (e.g. Karlsson et al. 1992). The SAM TLS will be able to measure (Sect. 4.2) the $\delta^{18} \mathrm{O}, \delta^{17} \mathrm{O}$, and $\delta^{13} \mathrm{C}$ in carbon dioxide and the $\delta^{18} \mathrm{O}, \delta^{17} \mathrm{O}$, and $\delta \mathrm{D}$ in water with precisions of 2 to 5 per mil both from the atmosphere and evolved from soil and rock samples. The EGA measurements may reflect the isotopic composition of an ancient atmosphere and also address the thermal and chemical conditions at the time these volatiles were trapped in minerals at the time of their formation in these ancient rocks.

Although sulfur-bearing minerals have been detected on the martian surface by orbiting spacecraft (e.g. Bibring et al. 2006a, 2006b, 2006c; Langevin et al. 2006) and landed missions (e.g. Wanke et al. 2001; Clark et al. 2005; Kounaves et al. 2010), as well as in martian meteorites (e.g. Farquhar et al. 2000; Greenwood 2000; McCubbin et al. 2009), no sulfurous gases have been detected in the martian atmosphere. Recent ground-based spectroscopic observations have placed an upper limit of $0.3 \mathrm{ppb}$ of $\mathrm{SO}_{2}$ in the current atmosphere (Encrenaz et al. 2011a, 2011b). Nevertheless, SAM will search for trace atmospheric constituents such as $\mathrm{SO}_{2}$ and $\mathrm{H}_{2} \mathrm{~S}$. The SAM QMS is expected to measure the major isotopes of $\mathrm{SO}_{2}$ evolved from solid samples containing $\mathrm{S}$ such as sulfates if these dissociate in the $950-1100{ }^{\circ} \mathrm{C}$ range of the SAM ovens. Analog studies and SAM FM calibration studies have determined that major isotopes may be measured by the QMS to $1 \%$ or better accuracy at percentage levels of sulfate mineral present in a sample in the absence of mass spectrometric interferences (Franz et al. 2011).

\subsection{Surface Mineralogy}

Since Mariner 9's discovery of fluvial valleys on Mars, supporting evidence for water-rock interactions has been sought from the surface mineralogy. Spectral observations from Earth hinted at secondary minerals such as phyllosilicates, sulfates, and carbonates in Martian regolith (e.g. McCord et al. 1982; Pollack et al. 1990; Blaney and McCord 1995). Martian meteorites contain minor ( $<1 \%$ by volume) clays, sulfates, halides, and carbonates (e.g. Bridges et al. 2001; Rao et al. 2005), and chemical data from all six Mars landers suggests that some aqueous minerals are widespread in soils (e.g. Clark and van Hart 1981; Wanke et al. 2001; Hecht et al. 2009; McSween et al. 2010). But to understand the environments that formed these minerals, they must be observed in geologic and stratigraphic context. 
At Gale crater, MSL will test the hypothesis that aqueous environments on Mars evolved from phyllosilicate-forming to sulfate-forming (Bibring et al. 2006a, 2006b, 2006c). The layered mound in Gale crater has hydrated sulfates and smectite clays that alternate stratigraphically (Milliken et al. 2010), consistent with a cyclical geochemical evolution. Curiosity can determine to what degree these minerals co-occur, or if they are segregated in distinct laminae. Crystalline red hematite is also observed in some Gale strata, distinct from the gray hematite in Meridiani and perhaps indicating a different diagenetic history-curiosity will explore this further via textural and compositional measurements.

Gale crater's sulfates are spectrally consistent with $\mathrm{Mg}$-rich varieties, but vary in their hydration from kieserite-like monohydrates $\left(\mathrm{MgSO}_{4} \cdot \mathrm{H}_{2} \mathrm{O}\right)$ to polyhydrated sulfates $\left(\mathrm{MgSO}_{4} \cdot \mathrm{nH}_{2} \mathrm{O}\right)$. Elsewhere on Mars, such varying sulfate hydration states have been interpreted as due to (1) differing water activity (or temperature) in the depositional environment (e.g. Roach et al. 2009), (2) diagenesis of polyhydrates to produce monohydrates at depth (e.g., Murchie et al. 2009a, 2009b), or (3) recent surficial hydration state changes (Mangold et al. 2008). The MSL team can test these hypotheses by better resolving hydration changes correlated to stratigraphy and/or with depth beneath the outcrop surface. Identifying the full mineral assemblage will also constrain conditions of formation-e.g., does jarosite accompany the hydrated sulfates as at Meridiani, or are Gale's sulfates consistent with less acidic fluids?

If phyllosilicates formed via alteration of basalt, this should have produced an abundance of cations $\left(\mathrm{Mg}_{2}{ }^{+2}, \mathrm{Ca}_{2}{ }^{+2}, \mathrm{Fe}_{2}{ }^{+2,3+}, \mathrm{Na}^{+}, \mathrm{K}^{+}\right)$. Leaching may have removed some of these cations as well as anions (e.g., $\mathrm{OH}^{-}, \mathrm{Cl}^{-}, \mathrm{SO}_{4}{ }^{2-}, \mathrm{CO}_{3}{ }^{2-}$ ) that could combine elsewhere to precipitate salts. The dominant anion(s) would reflect fluid chemistry and, in turn, the composition of contemporaneous volatile reservoirs, potentially including the atmosphere. Evaporite compositions may therefore yield insights into ancient cycling of $\mathrm{C}$ and $\mathrm{S}$. But most phyllosilicate spectra from Mars show little evidence for accompanying salts (Mangold et al. 2008; Milliken et al. 2009). At Gale crater, sulfates are present in some strata, but what of those beds in the lower mound with spectral evidence only for phyllosilicates (Milliken et al. 2010)? If carbonates are present here even at trace abundances, SAM's sensitivity should allow detection of these salts.

Finally, the Fe-rich smectites in Gale crater were once thought to be the most common type of phyllosilicate observed across Mars, but recent analysis suggests that clays in most other regions may instead be mixed-layer chlorite/smectites (Milliken et al. 2011). Diagenesis may gradually convert smectites into mixed-layer clays and ultimately chlorite, so the preservation of smectites over billions of years may strongly constrain the persistence of aqueous fluids (Tosca and Knoll 2009). This is especially true at Gale crater, where diagenesis should have occurred while the smectite-bearing rocks were buried beneath kilometers of overlying strata. Confirming the presence of smectites vs. chlorites based on chemistry and hydration will be an important activity for MSL.

The synergy of the MSL payload will enable an in-depth exploration of the diversity of minerals in Gale crater that is evident from orbit. A core objective of SAM is to search for organic compounds trapped in these minerals. However, whether or not organic compounds are present, the SAM EGA and isotope measurements are expected to provide data that is highly complementary to the elemental and mineralogical information provided by CheMin and other MSL instruments. 


\section{SAM Measurement Requirements, Design, and Capabilities}

\subsection{Overview of SAM Sample Processing and Gas Analysis Tools}

The SAM suite is designed to utilize a common set of solid sample and gas processing tools to service multiple instruments. This achieves not only an efficient use of resources, but also allows the three SAM instruments to analyze identical gas samples. A top-level view of the SAM sample processing flow to the gas analysis instruments is illustrated in Fig. 2 with a more detailed diagram of the SAM gas processing system and the individual instruments shown in Fig. 3.

SAM accepts either atmospheric samples or solid samples that are then processed to release gases that are analyzed by one or more of the three instruments of SAM. Gaseous samples can either be analyzed directly or after chemical enrichment and separation in the SAM GPS. Gases are extracted from solid samples by thermal processing in an inert helium gas flow, by thermal processing in an oxygen environment to combust refractory organics into carbon dioxide, or by wet chemical solvent extraction combined with chemical derivatization techniques. The SAM QMS, GC, and TLS can operate alone or in combination with one or two of the other instruments. Control of the SAM suite is achieved by a high level script language that allows the SAM technical and science team members to control the sequence of operations of the three instruments, the Solid Sample Inlet Tube (SSIT), the Sample Manipulation System (SMS), the Wide Range Pumps (WRPs), and the valves and heaters of the GPS.

\subsection{Overview of SAM Measurement Requirements and Capabilities}

The MSL mission goals summarized in Sect. 1 give rise to the five SAM science goals described in Sect. 2. These in turn give rise to the necessary data sets, performance requirements, and instrument and subsystem requirements detailed in Table 5. Section 8 illustrates performance realized by the SAM Flight Model (FM) for several of these requirements and Table 6 describes the major types of experiments that produce the required data. Typically a SAM experiment sequence of several hours duration on the surface of Mars will be required to implement several of the experiments listed in Table 6. The SAM Activity Dictionary developed for use by full MSL team reflects various options that may be exercised depending on environmental conditions and by energy resources available for SAM on that sol. Sections 4.3 through 4.7 describe the specifics of the measurement protocols developed to realize these requirements.

\subsection{Atmospheric Measurements}

The most frequent atmospheric measurement will be that designated AS-DIRECT in Table 6. This sequence has been exercised many times during SAM testing and calibration in the Mars chamber at the NASA GSFC. The QMS and TLS are evacuated by turning on WRP1 and WRP2 and opening microvalves to the TLS and the high conductance valve (HCV) to the QMS (Fig. 3). Background spectra for both of these instruments are then secured. The gas manifolds are brought to a temperature specified in the parameter file associated with the script and the entire manifold path from the gas inlet valve (V28) is cleaned by heating while being evacuated by WRP2. A typical manifold bake temperature is $135{ }^{\circ} \mathrm{C}$. The manifold is then isolated from WRP2 and gas introduced to the manifold by opening valve V28 that allows gas from outside the rover wall to be brought into SAM. QMS scans 
Table 5 SAM required data sets, performance, and implementation

\begin{tabular}{|c|c|c|}
\hline Required data sets & Required performance & Implementation \& performance \\
\hline $\begin{array}{l}\text { Inventory of volatile organic } \\
\text { compounds in rocks and soils } \\
\text { Signatures of refractory carbon } \\
\text { and its }{ }^{13} \mathrm{C} /{ }^{12} \mathrm{C} \text { ratio } \\
\text { Distribution of molecular weights } \\
\text { and chemical structures for organic } \\
\text { compounds }\end{array}$ & $\begin{array}{l}\text { Organics Analysis } \\
\text { - Volatile organics to } 1-10 \mathrm{ppb} \\
\text { (by mass) } \\
\text { - Polar and non-polar organics } \\
\text { (from saturated hydrocarbons } \\
\text { to carboxylic acids } \\
\text { - Complex organics to > }>20 \\
\text { C/molecule } \\
\text { - Refractory organics to ppm (by } \\
\text { mass) }\end{array}$ & $\begin{array}{l}\text { Gas Chromatograph Mass } \\
\text { Spectrometer } \\
\text { - Unit QMS mass resolution 2- } \\
535 \mathrm{Da} \\
\text { - } 6 \mathrm{GC} \text { columns to separate po- } \\
\text { lar and non-polar compounds \& } \\
\text { derivatized compounds (Table 9) } \\
\text { - Inert He carrier gas }\end{array}$ \\
\hline
\end{tabular}

Oxidation state distribution for organic compounds

Inventory of specific molecular classes (amino acids, amines, carboxylic acids)

\section{Atmospheric composition}

- Noble gas mixing ratios to $<15 \%$

- Major species \& trace species to ppm mixing ratios

- Ability to sample atmosphere at any time of day

\section{Mass Spectrometer}

- Dynamic range $\sim 10^{9}$

- Sensitivity $>10^{-2} \quad(\mathrm{cnts} / \mathrm{sec}) /$ (part/cc)

- 0.02 transition time to any $\mathrm{m} / \mathrm{z}$ value

- 0.1 Da scans for peak shape determination

- $25 \mathrm{Da}$ band scans to capture GC transients

Inventory and temperature profile of volatile inorganic compounds released from solids

Methane abundance and its variation in the atmosphere and its ${ }^{13} \mathrm{C} /{ }^{12} \mathrm{C}$ ratio

Seasonal and diurnal variation of major and minor atmospheric species

Atmospheric mixing ratios of noble gases $\mathrm{Xe}, \mathrm{Kr}$, Ar, and $\mathrm{Ne}$ Isotopic composition of atmospheric noble gases $\mathrm{Xe}, \mathrm{Kr}$, $\mathrm{Ar}$, and $\mathrm{Ne}$

\section{Isotopic Analysis}

- $\delta^{13} \mathrm{C}$ to 5 per mil in $\mathrm{CO}_{2}$ and 10 per mil in $\mathrm{CH}_{4}$

- $\delta^{18} \mathrm{O}$ to 5 per mil in $\mathrm{H}_{2} \mathrm{O}$ and $\mathrm{CO}_{2}$

- $\delta^{17} \mathrm{O}$ to 10 per mil in $\mathrm{H}_{2} \mathrm{O}$ and $\mathrm{CO}_{2}$

- Noble gas isotopes from $<10$ 20 per mil

- C isotopic composition of refractory organics by combustion to $\mathrm{CO}_{2}$

Evolved Gas Analysis

- Mineral classes (carbonates, sulfates, clays, etc.) with ppm sensitivity

- Features (e.g. peak T) in EGA profiles to $\pm 20^{\circ} \mathrm{C}$
Tunable Laser Spectrometer

- Separate methane (IC) and $\mathrm{CO}_{2}$ (NIR) lasers

- Wavelength shift in NIR laser for $\mathrm{H}_{2} \mathrm{O}$ isotopes

- Performance given in Table 10

Gas \& solid processing

- Turbomolecular pumps

- Heated transfer lines $\left(T \geq 135^{\circ} \mathrm{C}\right)$

- Controlled solid sample heating ambient to 900 to $1100{ }^{\circ} \mathrm{C}$ in inert quartz cups

- Solvent extraction \& derivatization

- Combustion with $\mathrm{O}_{2}$

- Getters \& scrubbers to remove selected gases

begin after gas from the manifold is introduced to the ion source of the mass spectrometer through the glass capillary inlet associated with valve V11. The pressure in the ion source of the mass spectrometer is established by the dynamic balance between the gas flow through the capillary and WRP1. Typically in the QMS atmospheric measurement sequence, 0.1 Da mass scans will be implemented for part of the scan time to improve the precision of the measurement.

Several different pressures of gas are generally sampled in the TLS to insure that a wide enough gas density range is sampled to avoid a saturated signal for any absorption line of interest. TLS measurements are always implemented in a static mode where the gas in the Herriot cell is isolated by valves V8, V9, and V10. Atmospheric samples can be introduced to the TLS either through V28 or through its dedicated inlet V10. 
Table 6 Solid sample, atmospheric, or special processing experiments

Solid Samples (SS) Experiment description

SS-EGA Evolved Gas Experiment-sample in quartz cup heated to 900-1100 C while QMS scans relevant $m / z$ values

SS-GCMS Gas Chromatograph Mass Spectrometer analysis_-gas trapped during a portion of the SS-EGA or SS-DERIV experiments is analyzed by GCMS

SS-TLS Tunable Laser Spectrometer analysis-gas trapped in TLS during SS-EGA is analyzed for abundance and isotopes

SS-COMBUST Combustion experiment-sample in quartz cup is heated in oxygen and $\mathrm{CO}_{2}$ produced introduced into the TLS for isotope analysis

SS-CH_EXT Chemical Extraction and Derivatization-foil on wet chemical lab contained in sealed metal cup is punctured and solid sample introduced. After heating and introducing vapor to SAM trap SS-GCMS experiment is carried out

Atmos. samples Experiment description

AS-DIRECT Atmospheric gas introduced into SAM manifolds and analyzed by QMS and/or TLS

AS-NG Noble gas analysis is implemented by scrubbing reactive gases and introducing purified noble gases into the QMS in static mode

AS- $\mathrm{CH}_{4} \quad$ Methane is enriched in GPS for more sensitive TLS methane measurements

AS-ENR Atmospheric enrichment-GPS processes volumes of gas for enrichment of trace atmospheric species for either QMS or GCMS analysis

Special processing

Includes experiment sequences to regenerate getters and scrubbers, bake manifold lines and QMS, and precondition a sample cup for subsequent SS-EGA experiment

$A S-N G$ (the noble gas atmospheric experiment) is designed to remove chemically active gases from the mixture introduced into the manifold for higher precision and more sensitive measurement of noble gas ratios and the isotopic distribution in $\mathrm{Ne}, \mathrm{Ar}, \mathrm{Kr}$, and $\mathrm{Xe}$. The combination of the scrubber and getter illustrated in Fig. 3 efficiently removes all gases except for the noble gases and methane, which is not efficiently removed by the getter. Argon and neon are measured following this gas separation by introduction into the mass spectrometer through a higher conductance capillary leak associated with V12. Further enrichment of the trace noble gases $\mathrm{Kr}$ and $\mathrm{Xe}$ is achieved by their separation from the Ar and $\mathrm{Ne}$ by trapping on the SAM trap (HCT) cooled by a dual stage thermo-electric cooler (TEC). Following the adsorption of $\mathrm{Kr}$ and $\mathrm{Xe}$ on the carbosieve element of this trap, the gasses not adsorbed are pumped and the $\mathrm{Kr}$ and Xe then released from the HCT into the manifold. The QMS is then isolated from WRP1 by closing HCV1 and a portion of the trace noble gases in the manifold introduced to the QMS through the capillary inlet associated with V12. After several mass scans have been secured, the pressure in the QMS is increased by introduction of additional $\mathrm{Kr}$ and $\mathrm{Xe}$ from the manifold and the scans repeated until the trace ${ }^{124} \mathrm{Xe}$ and ${ }^{126} \mathrm{Xe}$ have been measured with sufficient signal to noise. AS-NG will be fully optimized on the SAM testbed before its use on Mars.

$\mathrm{AS}-\mathrm{CH}_{4}$ is similarly designed to increase the density of methane in the TLS above the value that can be realized by direct introduction of atmospheric gas into the TLS. In this experiment the major atmospheric constituents $\mathrm{CO}_{2}$ and $\mathrm{N}_{2}$ are scrubbed from the gas in the manifold using first the scrubber and then the getter. The major constituent of the remaining gas in the manifold is $\mathrm{Ar}$, mixed with the trace amounts of $\mathrm{CH}_{4}$ that were not removed by the 
gas scrubbing system. This mixture is then introduced to the evacuated TLS by opening the valves between the getter/scrubber manifolds and the TLS. The entire process is repeated to build up the methane density in the TLS and reduce the sensitivity threshold for detection and isotope measurements.

$A S$-ENR is an experiment that will enrich $\mathrm{H}_{2} \mathrm{O}$ for more precise $\mathrm{D} / \mathrm{H}$ measurements and also to search for trace atmospheric species that might adsorb more strongly on the scrubber than the major atmospheric constituent $\mathrm{CO}_{2}$. After atmospheric gas flows over the $\mathrm{CO}_{2} / \mathrm{H}_{2} \mathrm{O}$ scrubber for a preselected time period these traps are heated to release gas into either the TLS for D/H analysis or onto the hydrocarbon trap if SAM is implementing a search for higher molecular weight atmospheric species.

\subsection{Solid Sample Measurements of Evolved Gas}

SS-EGA is the core SAM solid sample experiment with 59 of the 74 cups dedicated to this experiment. Approximately $0.078 \mathrm{cc}$ of powdered sample sieved to $\leq 150 \mu \mathrm{m}$ is released from the Curiosity Sample Processing and Handling $(\mathrm{SPaH})$ system by opening a door that delivers this material to a SAM inlet funnel. At the time of delivery, the command sequence has enabled the funnel and transfer tube of the SAM inlet to vibrate utilizing a piezoelectric actuator. This motion facilitates the transfer of sample into the quartz cup, which is then sealed in its oven for the evolved gas analysis within several minutes. The solid sample analysis sequence consists of first thermally conditioning the SAM manifolds that will be exposed to the evolved gas and securing background spectra from both the QMS and the TLS. A helium flow of approximately $0.03 \mathrm{~atm}-\mathrm{cc} / \mathrm{sec}$ is then initiated to sweep gases evolved from the sample past the capillary inlet of the mass spectrometer and into the TLS at selected sample temperature intervals. The flow of helium alone maintains the pressure in the manifold between the flow restrictor out of the helium tank and the flow restrictor associated with vale V20 at approximately 30 millibar. After bringing the sample to approximately $125^{\circ} \mathrm{C}$ for a script-selected period of time (typically $\sim 10$ minutes) the sample temperature is ramped up to its final temperature $\left(950{ }^{\circ} \mathrm{C}\right.$ to $\left.1100{ }^{\circ} \mathrm{C}\right)$ while the QMS is continuously scanned. Over most of the low and mid temperature range the temperature ramp is nearly linear with a nominal value of $35^{\circ} \mathrm{C} /$ minute. Above $\sim 800^{\circ} \mathrm{C}$ the oven power supply begins to apply its maximum possible power to the heater wire of the oven and the temperature vs. time curve begins to become non-linear. The final temperature the sample in the oven can reach will depend on the duration selected for the ramp and the oven selected. One of the ovens incorporates an auxiliary heater designed to bring the sample temperature to $1100{ }^{\circ} \mathrm{C}$ instead of the $950{ }^{\circ} \mathrm{C}$ normally realized in the other oven.

At selected intervals during the evolved gas temperature ramp the gas flow can either be diverted through the SAM HC trap for later GCMS analysis or directed into the TLS for later $\mathrm{CO}_{2}, \mathrm{H}_{2} \mathrm{O}$, and $\mathrm{CH}_{4}$ abundance and isotope measurements.

The solid sample gas chromatograph mass spectrometer analysis (SS-GCMS) will normally be performed immediately following the SS-EGA sequence. In SS-GCMS the manifold is pressurized to nearly one bar of helium utilizing either V34 or V44 from one of the two helium tanks (Fig. 3). After purging the selected GC column, organics are thermally released from the SAM HC trap and trapped on the smaller trap (IT) in line with the selected GC column or directly injected in the dedicated GC column (3 of the 6 GC columns don't have ITs). In case of IT trapping, rapid heating of the GC IT marks the start of the GC chromatogram and the eluted compounds are detected both with the thermal conductivity detector (TCD) of that column, and by the fraction of gas diverted into the mass spectrometer. The flow restrictor into the mass spectrometer consists of a laser drilled hole in a metal disc 
surrounded by a machined and sintered metal filter that serves to restrict the flow of compounds that are heavier than the helium carrier gas. This flow restrictor split design prevents the vacuum of the mass spectrometer with its miniaturized pump from being overloaded with carrier gas while still maintaining the required sensitivity for organics detection.

TLS analysis of the gas trapped at selected sample temperature intervals is normally carried out after the completion of the SS-GCMS analysis. The SS-TLS sequence utilizes the preselected laser or lasers. Nominally, with sufficient energy available from the MSL battery, scans of all three molecules of interest and their isotopes would be implemented. Since the species of interest may be present in the TLS in widely differing ratios, line saturation effects are avoided by implementing several cycles of pressure reduction in the cell to bring possibly saturated peaks into the desired measurement range.

Measurement sequences SS-EGA, SS-GCMS, and SS-TLS are normally implemented sequentially by a script that takes 4-6 hours to run depending on the environmental conditions, battery energy available, and SAM options selected for the three instruments and the GPS such as manifold temperatures, number of TLS lasers, integration times, and post experiment clean up durations. If the MSL available energy is limited on a particular sol these three experiments may also be implemented on sequential sols. SS-EGA is normally preceded by a sequence that cleans an empty cup to a temperature above $900{ }^{\circ} \mathrm{C}$ with occasional bursts of helium sent through the manifold lines to remove background gases that may have accumulated on the cup. The cup is then left in the oven in a clean state until a following sol when SA/SPaH is ready to be utilized to deliver sample to SAM. It is expected that the sol after sample delivery would normally be dedicated to the SS-EGA, SS-GCMS, and SS-TLS sequences.

\subsection{Solid Sample Measurements Based on Combustion}

The SS-COMBUST experiment utilizes $\mathrm{O}_{2}$ from an onboard SAM reservoir to combust the more refractory component of carbon that might be present in a sample and transform it into $\mathrm{CO}_{2}$. After a period of combustion at temperatures in the $700-950{ }^{\circ} \mathrm{C}$ range, the gaseous products are introduced to the pre-evacuated TLS where the $\delta^{13} \mathrm{C}$ in $\mathrm{CO}_{2}$ is measured. There is the potential for mixing of the $\mathrm{CO}_{2}$ from the combusted carbon with $\mathrm{CO}_{2}$ evolved from minerals if the sample contains carbonates. SS-EGA experiments and CheMin mineralogical characterization will be used to estimate the contribution of carbonate carbon to the bulk $\delta^{13} \mathrm{C}$ of a sample. This will help determine whether a combustion experiment on a mixed sample heated just past the temperature where $\mathrm{CO}_{2}$ is evolved from carbonates can still provide useful isotopic information. The degree to which a mass balance approach can be used to isolate $\delta^{13} \mathrm{C}$ of the organic fraction of the sample will be assessed with testbed experiments of relevant analogues involving separate pyrolysis and combustion runs of the same sample to give separate $\delta^{13} \mathrm{C}$ values representing the inorganic (pyrolysis) and total, or bulk (combustion) fractions. All testbed measurements will be validated using conventional isotope ratio mass spectrometer protocols where the combustion is preceded by acid dissolution of the carbonates.

\subsection{Solid Sample Measurements Based on Wet Chemical Processing}

The search for astrobiologically relevant organic compounds on Mars, including key molecules such as amino acids and carboxylic acids, will require a wet chemical processing and derivatization to transform these organic compounds into species that are sufficiently volatile and amenable to GC columns to be detected by GCMS (Meunier et al. 
2007; Buch et al. 2009). Chemical derivatization using dimethylformamide-dimethylacetal (DMF-DMA) has already been incorporated into the Cometary Sampling and Composition (COSAC) evolved gas experiment on ESA's Rosetta Lander and will provide amino acid detection and enantiomeric measurements on the surface of comet 67P/ChuryumovGerasimenko in 2014 (Meierhenrich et al. 2001; Szopa et al. 2003; Goesmann et al. 2007). The SAM instrument suite on MSL is designed to detect a wide range of chemical signatures, organic and inorganic, that could provide evidence of a habitable environment and possibly pointers toward life (Cabane et al. 2002; Mahaffy et al. 2010). The SAM instrument can perform a lower temperature $\left(75-300{ }^{\circ} \mathrm{C}\right)$ wet chemical processing step prior to GCMS analysis using one of seven sealed metal cups containing a mixture of N-methyl-N-(tertbutyldimethylsilyl) trifluoroacetamide (MTBSTFA) and DMF that will target less volatile and thermally less stable organic compounds such as amino and carboxylic acids that cannot be readily detected by high temperature pyrolysis and GCMS analysis alone (Fig. 1A). SAM also has the ability to detect polar molecular components that are bound into more complex organic molecules (e.g. amino acids in proteins, fatty acids in membranes, or carboxylic acids in abiotic macromolecules) by thermochemolysis (i.e., specifically, thermally assisted hydrolysis and methylation) at temperatures $>340{ }^{\circ} \mathrm{C}$ (Kaal and Janssen 2008; Geffroy-Rodier et al. 2009) using a mixture of tetramethylammonium hydroxide (TMAH) and methanol (Fig. 1B).

The nine metal wet-chemistry cups used in SAM are located in the outer ring of the Sample Manipulation System (SMS) carousel (Fig. 2). Each derivatization cup contains two separate foil capped reservoirs (outer and inner volumes) that are filled with reagent fluids and an internal reaction standard, respectively. The outer volumes of seven of the nine metal cups contain $0.5 \mathrm{~mL}$ of a 4:1 mixture of MTBSTFA (Sigma-Aldrich, $97 \%$ purity) and DMF (Pierce, >99\% purity) containing $25 \mathrm{nmol}$ of pyrene (Sigma-Aldrich, >99\% purity) that was injected through the pinch-off tube with a syringe pump. After filling, each cup was subjected to three freeze-pump-thaw cycles in order to degas the solvents and the outer reservoir was subsequently sealed via the pinch-off tube. The inner volumes of these seven derivatization cups were filled with $40 \mathrm{nmol}$ of 3-fluoro-DL-valine (Fluka, >99\% purity, hereafter, $3-\mathrm{FV})$ by injecting $50 \mu \mathrm{L}$ of a stock 3 -FV solution $\left(1 \times 10^{-3} \mathrm{M}\right)$ prepared by dissolving solid 3-FV in Millipore water. After loading, the 3-FV solution was evaporated to dryness inside the inner volume under vacuum and hermetically sealed under partial vacuum by pinch-off. 3-FV was selected as an internal reaction standard for the MTBSTFA derivatization experiment since this amino acid will readily react with MTBSTFA when exposed to the solvent after foil puncture and is not expected to be prevalent on Mars. The other two metal wet-chemistry cups in the SMS were loaded with $0.5 \mathrm{~mL}$ of TMAH and methanol (Sigma-Aldrich, $25 \%$ in methanol, product \#334901) containing $34 \mathrm{nmol} 1$ fluoronaphthalene (Sigma-Aldrich, 99.0\%, product \#196657) and $25 \mathrm{nmol}$ pyrene (dried from Sigma-Aldrich, Riedel de-Haën-OEKANAL, analytical standard, product \#36944) in the outer reservoir plus $13 \mathrm{nmol}$ of nonanoic acid (Sigma Aldrich, $97 \%$, product \#N5502) in the internal reservoir and then sealed by pinch-off with the same procedure used for the MTBSTFA derivatization cups. Nonanoic acid was selected as an internal reaction standard due to the high volatility of its methyl ester derivative. 1-fluoronaphthalene and pyrene are TMAH recovery standards which should not undergo significant reaction.

The steps required for chemical processing of a solid sample on Mars are illustrated in Fig. 2. Prior to receiving a solid sample from the MSL SA/SPaH system, the foil on a metal wet chemistry cup is punctured by raising the cup under a long-puncture pin located on the outer ring of the SMS. This step allows the derivatization fluid from the outer reservoir to begin reacting with the dry internal standard in the inner volume. The cup is 
then rotated into position directly underneath the solid sample inlet tube where the solid sample is delivered and the cup is raised into the SAM pyrolysis oven and sealed to it by a copper o-ring knife-edge seal at the base of the cup stem. For the MTBSTFA derivatization experiment, excess solvent will be pumped out of the SAM exhaust vent, after an initial thermal incubation. The remaining derivatized products will then be driven to the SAM hydrocarbon trap by heating the oven to elevated temperatures under He carrier gas at a flow rate of $\sim 0.03$ atm-cc/sec. For the TMAH thermochemolysis experiment, venting of excess reagents and unwanted reaction byproducts can occur after passing products through the SAM hydrocarbon trap. Volatile MTBSTFA or TMAH derivatives trapped on the SAM hydrocarbon trap are then released from the trap at $250{ }^{\circ} \mathrm{C}$ under $\mathrm{He}$ carrier gas flow and sent to one of six GC columns for separation and mass identification by the SAM QMS. The derivatization extraction efficiency of any organics identified in the sample will be estimated by a comparison of the peak area of the derivatized internal standard (3-FV or nonanoic acid) with the peak area observed in the procedural blank run. Since derivatization experiments were not tested on the SAM flight instrument in order to keep SAM as clean as possible before its first experiments on the surface of Mars, optimization of this experiment and clean up sequences will be performed on the ground using an identical SAM test-bed instrument.

\subsection{In Situ Calibration Capabilities}

SAM has the ability to implement in situ calibration of all three instruments through the use of either a gas sample from its on-board calibration cell or by utilization of one of the six individual metal calibration cups on the SMS. The solid and gas calibration compounds and the requirements for their utilization are described in Sects. 6.12 and 6.13 respectively. The timing and frequency of these in situ calibration experiments will be determined during the course of the landed mission by the science team.

\section{SAM Surface Operations Plan Overview}

SAM's experiments are designed to address the goals and objectives described in Sect. 2. SAM's flexible scripting enables a synergistic approach to discovery as the mission progresses and the science operations working group (SOWG) becomes more practiced in its ability to employ the payload to address complex questions. For example, the SAM evolved gas interrogation is sensitive to volatiles in mineral phases and can work together with CheMin, ChemCam and APXS to provide a comprehensive picture of the mineralogical and geochemical character of the rock and soil samples that are studied.

The SAM science team will participate in both tactical and strategic planning on every day of the mission. The team's scientific input will be through the MSL science theme groups (STG): Volatiles and Isotopic Chemistry, Inorganic Geochemistry and Mineralogy, Geology and Atmosphere and Environment, according to the interests and expertise of the co-investigators and collaborators. There are also a few SAM-specific roles. Both MSL and SAM roles are summarized in Table 7.

The mission operations concept includes a broad division of activities into six sol types as shown in Table 8. SAM experiments are conducted during the analytical sols, however the SAM team participates in operations during every sol type. The sol types were conceived for planning purposes, and as the mission progresses there may be hybrids with various combinations of activities. 
Table 7 MSL and SAM specific roles for the SAM science team

\begin{tabular}{ll}
\hline MSL role & Description \\
\hline SOWG Chair & $\begin{array}{l}\text { Meets with the SOWG documentarian, LTP, science planner, and STLs, if necessary } \\
\text { during downlink assessment. Leads meetings, and helps the SOWG reach consensus }\end{array}$ \\
& $\begin{array}{l}\text { regarding the activity plan for the next sol and adjudicates when consensus is not } \\
\text { reached. Works with the Integrated Planning and Execution element to ensure that the } \\
\text { sequences generated express the SOWG intent }\end{array}$
\end{tabular}

Documentarian Records all discussions during the Science Kickoff meeting and SOWG meetings, or any other meetings with the SOWG chair

Long Term Has a strategic and a tactical role, reporting at each SOWG meeting, reviewing future

Planner plans, results of recent sols, recording progress against metrics, reporting on the current sol path/sol tree, notifying the group of upcoming restrictions on vehicle use, resource availability and data volumes either acquired or upcoming

STG Lead Coordinates theme group discussions and activity planning. Submits an activity request at the SOWG meeting. STL presents the findings and implications from their theme group and advocates for their activity request

STG member Works in a group to analyze data, test hypotheses and prepare activity requests

SAM role Description

Payload Uplink Lead (PUL)

Payload Downlink Lead (PDL)

Script Czar

Test Bed Lead

Test Bed

Scientist

SAM Systems

PDS Archivist

Payload

Element Lead (PEL)
Provides advice and clarification on SAM and its activities. Develops command sequences based on the approved activity plan. Prepares SAM parameter tables and when necessary uploads new SAM scripts

Assess downlink and prepare higher-level products for distribution to the entire SOWG. Reports on instrument health, consumable usage, and integrity of the science data

Expert on the development, debugging and modification of SAM scripts and parameter tables

Expert in the operation and instrument health of the SAM high fidelity test bed Leads the experiments on the test bed is expert in the interpretation of data collected from TB SAM

Systems engineering lead follows the performance of both flight SAM and test bed SAM Librarian for SAM data and preparation of data for storage not only at the NASA GSFC but also for PDS

There will be a PEL for the QMS, the TLS, and the GC who will be the designated expert on that instrument as questions arise from the larger MSL team

The SAM analytical sol actually consists of at least three sols consisting of (1) sample cup conditioning, (2) sample delivery to cup and isolation of cup in oven, and (3) sample processing. This is necessary because the activities that comprise a full solid sample experiment are energy intensive. Parts of the SAM experiment, such as pre-conditioning a sample cup, must be executed before a sample is portioned and delivered because the cup must be pristine. Once powdered, the sample must be delivered into a waiting cup as soon as possible to reduce loss of volatiles from the rock. In the case of sols where there is either insufficient energy to run TLS as well as QMS and GC, or it is too hot to do so, SAM may exercise the option to ingest the evolved or atmospheric gas into the TLS and run the TLS analysis on the following sol. SAM may conduct atmospheric experiments over more than one closely spaced sols in order to capture atmosphere at different times of the diurnal cycle and still stay within the energy budget. 
Table 8 MSL sol types and SAM team activities

Sol type SAM team activity

Reconnaissance Study camera images, Chemcam target data and REMS and RAD data. In a strategic sense, the team can begin planning solid sample or atmospheric experiments

Approach DAN, REMS, RAD data are collected; cameras and Chemcam provide data for tactical planning. SAM team reviews data and participates in STGs

Contact SAM science team reviews contact data and participates in decision whether or not to drill a rock interrogated by contact instruments. If a decision is made to drill or to scoop soil, SAM must pre-condition a cup prior to drilling and sample delivery

Drilling SAM will receive a sample as soon as it is ready for portioning, and then the sample will be transported to the appropriate pyrolysis oven where it will be sealed. The team will plan for the next day's analysis, paying careful attention to REMS data and other thermal information. The SAM PUL will prepare the parameters for the SAM script to be run and also prepare the sequence to call the SAM experiment for the next sol. It is also possible that an additional sol might be spent examining a drilled or scooped sample with contact instruments before SAM and CheMin ingest the sample

Analysis SAM experiments are conducted. Depending upon the thermal environment and the energy required (or available), SAM may take two days to perform the analysis. For a solid sample experiment SAM might pyrolyze sample on one sol and analyzed the next. Or in the case of atmospheric experiments where SAM must sample at different times of the sol, SAM might require two or three consecutive sols to sample the complete diurnal cycle. On analytical sols where CheMin conducts experiments, SAM science team members participate in review of the data with the rest of the SOWG while also planning for the SAM experiments

Traverse (rover SAM team plans science with the STGs and analyzes untargeted camera and ChemCam travels $>100 \mathrm{~m}$ ) data, as well as REMS, RAD and DAN data

\section{SAM Suite Elements}

\subsection{Solid Sample Inlet System}

The SSIT (Fig. 4) consists of dual inlet funnels and tubes that direct the volume of sample released from the Curiosity sample processing system into the SAM SMS cups. The funnels and tubes are mounted on a bellows support vibrated as the sample is delivered to maximize the mass of sample delivered to the cup without adhering to the walls. The redundant piezoelectric actuators drive a frequency sweep through a series of mechanical resonances from 100 to $500 \mathrm{~Hz}$ over a period of 15 seconds resulting in a peak vibration amplitude of $0.22 \mathrm{~mm}$. The tube portion of the SSIT can be heated to $120^{\circ} \mathrm{C}$ and the funnel portion more exposed to the ambient environment to $120^{\circ} \mathrm{C}$. The requirement of robust transport of $98 \%$ of delivered sample was verified by using a variety of powdered ( $<120$ micron) analog samples such as kaolinite, goethite, JSC-Mars-1, and iron(III) oxide dropped through the SSIT at Mars pressures at a variety of angles. In order to accommodate the largest particles that might be expected from the $\mathrm{SA} / \mathrm{SPaH}$ scoop that would have passed through a $1 \mathrm{~mm}$ square mesh, the SSIT inlet tubes were sized to $4.1 \mathrm{~mm}$ inside diameter.

\subsection{Sample Manipulation System}

The SMS is an electromechanical system with robotic features integral to the SAM instrument. The SMS is an under-actuated ( 2 actuators, 3 degrees-of-freedom) system. It is responsible for controlled manipulation of the sample from SAM's sample inlet device to 
Fig. 4 The two SAM inlet funnels and tubes that transport sample to the SMS cups are shown and the position of the actuators and heaters shown. The height of the SSIT from the top of the funnel to the bottom of the sample delivery tube is $25.4 \mathrm{~cm}$

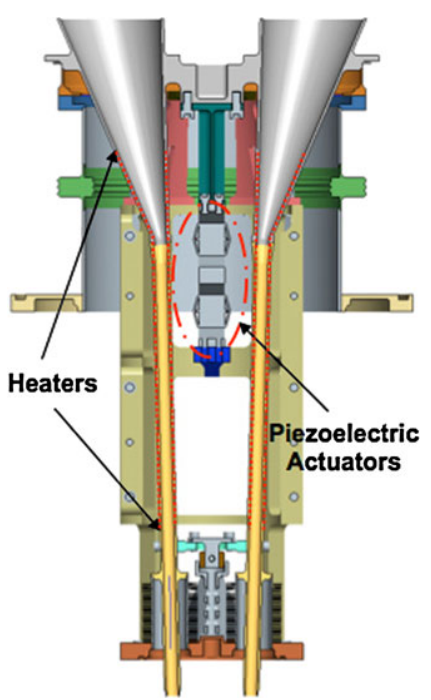

Fig. 5 The left pane shows the SMS integrated to SAM and the right pane shows both metal and quartz cups on the sample carousel
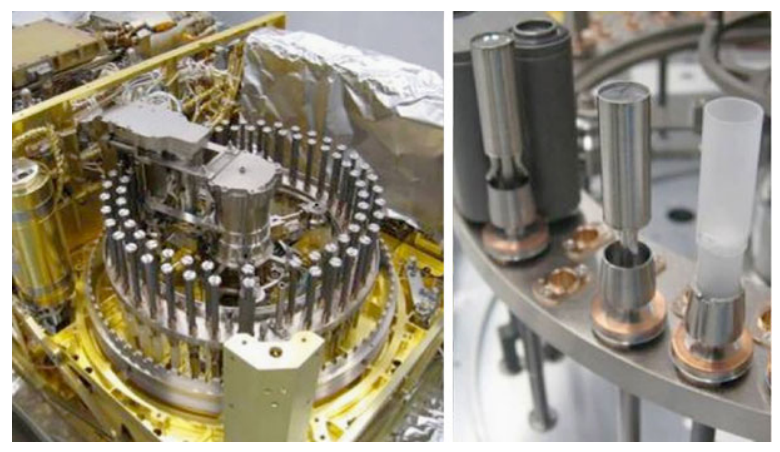

ovens, where the solid samples are pyrolyzed. The SMS also has a resettable launch lock, mechanized contamination seal, and the capability to puncture metallic foil seals for wet chemistry experiments.

SMS Driving Requirements: The SMS, Fig. 5, accepts solid material from the SA/SPaH delivered through one of the two SSIT's into any of 74 sample cups and transports the selected sample cup to a pyrolysis oven. The SMS must position each cup to within $0.71 \mathrm{~mm}$ true position at multiple interfaces including the solid sample inlet device, foil puncture stations for sealed metal cups, and a pyrolysis oven on each row of the sample carousel. Once the SMS inserts the sample cup into the oven, it creates a seal force sufficient to provide a leak tight $\left(1 \times 10^{-5} \mathrm{cc} \mathrm{He} / \mathrm{sec}\right)$ seal. To allow SAM to re-use sample cups the SMS applies seal force with an accuracy of $\pm 10 \%$ to seal a sample inside an oven. A seal is created via an annealed copper disk brazed to the sample cup and a titanium knife-edge on the oven. Each successive use of a sample cup requires a larger seal force up to a maximum force of 1350 Newtons.

Contamination concerns led to implementation of a vacuum seal to prevent contamination of the interior of the SMS during assembly, test, and launch operations. The outgassing rate of the SMS was required to be less than $4.2 \mathrm{E}-14 \mathrm{~g} / \mathrm{cm}^{2} / \mathrm{sec}$ as measured by a thermally controlled quartz crystal microbalance (TQCM). Molecular contamination of the sample 

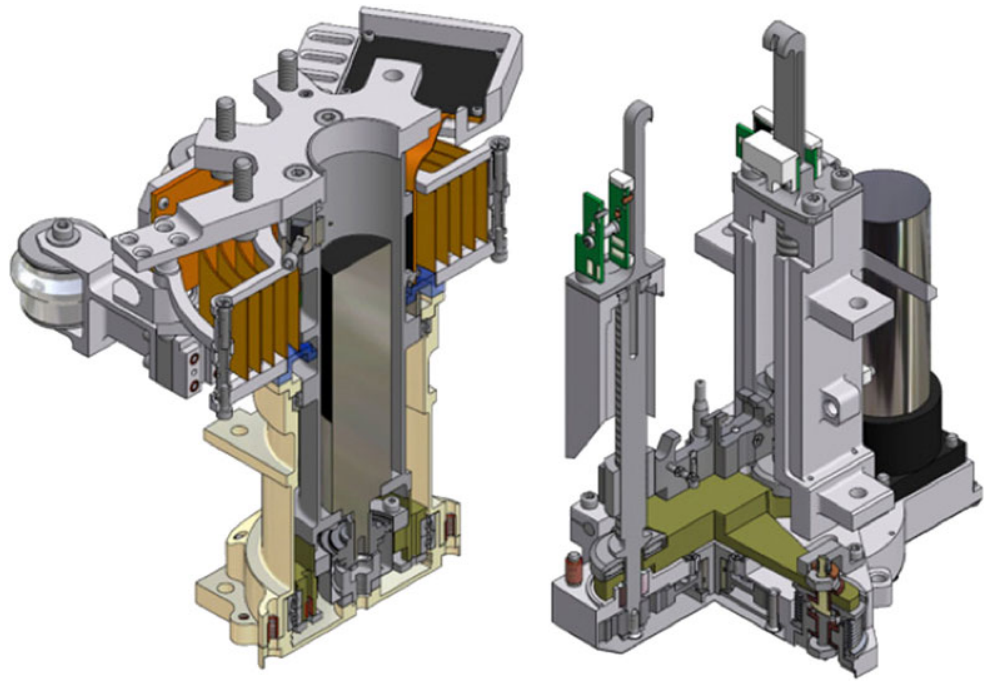

Fig. 6 The SMS actuators: (left) carousel; (right) elevator

from transport of molecular species contained in the SMS itself is minimized by leaving the cup in the clean oven after preconditioning it to above $900{ }^{\circ} \mathrm{C}$ and by rapidly moving it to the end of the SSIT when sample is ready for delivery and then rapidly back to its clean station in the oven.

The SMS consumes less than 5 watts continuous and 10 watts peak power. It is capable of recovering from an unexpected power loss through feedback devices and a robust high level control architecture that combined provide full fault detection and correction capability.

SMS System Design Overview: The SMS is comprised of a high reduction, low backlash actuator, a compliant linear actuator, restraint/release devices, a launch restraint, and a custom incremental encoder. Figure 6 depicts the two actuators, carousel and elevator, designed for the SMS. The carousel actuator rotates the sample carousel and the elevator mechanism. The elevator actuator raises and lowers sample cups at the pyrolysis ovens and sample inlet.

Carousel Drive: The carousel drive is comprised of a hall commutated brushless DC motor, 25:1 planetary gearbox, and 100:1 harmonic drive for an overall gear reduction of 2500:1 and 30000 counts per revolution (Fig. 6, left).

The carousel drive is used to position both the sample cup elevator and the sample carousel. Two degrees of freedom are achieved via one actuator. When positioning a sample cup, the carousel actuator is coupled to the sample carousel. In this position the sample cup elevator position is fixed with respect to the sample carousel. Once the sample cup is in position, the sample carousel is locked to ground via a spring-energized latch and the carousel actuator rotates the sample cup elevator relative to the sample carousel. This is necessary to position the elevator beneath the cup to be used in the experiment.

Elevator drive: The elevator drive, Fig. 6 (right panel) raises and lowers cups, provides the force required to seal cups in pyrolysis ovens, and provides a resettable launch lock for the elevator mechanism. The linear elevator drive consists of a hall commutated brushless DC motor, 80:1 planetary gearbox, 2.4:1 spur gear arrangement, and a pair of 5/16-12 ACME lead screws, resulting in resolution of 1088 hall counts per millimeter of travel. The elevator actuator delivers up to 1770 Newtons to a sample cup within $\pm 10 \%$ of the commanded force. 
Fig. 7 The fully populated SAM carousel

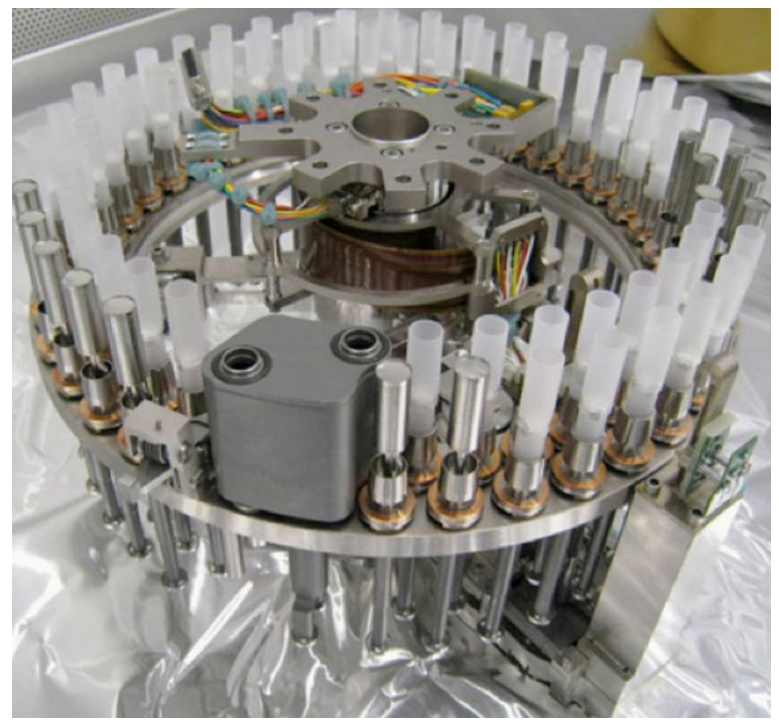

Sample Cup Carousel: The SMS accommodates 74 sample cups packaged in two polar arrays and an excess sample reservoir that also provides a contamination seal for the SMS interior. The packing density of the cups on the carousel was maximized to provide as many clean cups as possible within the allotted volume as well as provide the optimal safe distance between cups. The 74 sample cups (Fig. 7) are separated into three categories: solid sample quartz cups (59), foil topped metal cups for wet chemistry experiments (9), and foil topped metal cups containing calibration sample (6).

SMS Summary: The SMS is a precision sample delivery system designed to operate for years in the extreme temperatures and dry atmosphere of Mars. The SMS provides all the necessary precision sample manipulations required by the SAM instrument suite with the requisite reliability.

\subsection{SAM Ovens and Cups}

The SAM oven (Fig. 8) was designed to bring the sample to a maximum temperature of 900-1100 ${ }^{\circ} \mathrm{C}$ to release volatiles for QMS, TLS, and GCMS analysis. The quartz or metal cup containing respectively dry sample or wet reagents combined with sample is positioned by the SMS elevator mechanism into the center of the oven whose walls are an $0.096 \mathrm{~cm}$ Inconel 693 tube. The platinum-zirconium alloy $0.51 \mathrm{~mm}$ heater wire was threaded up and down through holes formed in an alumina insulator. A small getter pumps the vacuum housing that contains the heater wire and the Rhenium-coated precision-fabricated molybdenum radiation shields. The getter was activated with an external oven prior to installation of the oven in the suite following repeated cycles of outgassing of the materials of the oven. It was then pinched off to create a vacuum designed to be maintained for the duration of the mission. The cleats shown at the base of the oven mate with cleats on the SMS to locally react the force of sealing the metal knife edge against the copper seal brazed to the cup stem. A force of $667 \mathrm{~N}$ is utilized for a fresh copper seal and an incremental force of $111 \mathrm{~N}$ utilized for each subsequent seal up to the $1350 \mathrm{~N}$ limit at which point the seal force is kept constant. Testing showed acceptable leak rates were repeatably achieved at $1350 \mathrm{~N}$ without successively incrementing the force. The leak requirement on the seal was $1 \times 10^{-5}$ atm cc/sec-He. 

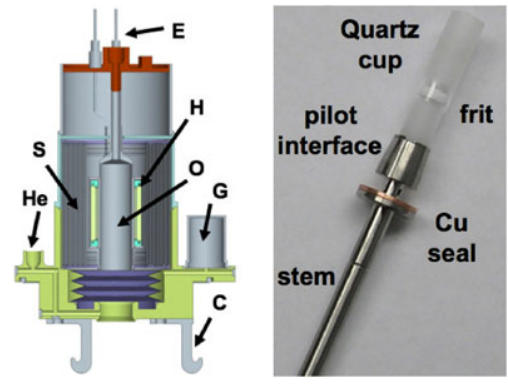

Fig. 8 The left pane illustrates the SAM oven design with the radiation shields $(S)$, the heater wire $(H)$ isolated with alumina, the reaction clamps $(C)$, the getter $(G)$, the electrical feedthrough pins $(E)$, the oven $(O)$, and the helium carrier gas inlet $(\mathrm{He})$. A quartz sample cup is shown at right that would be inserted into this oven from below and hermetically sealed. For reference the inside diameter of the oven at the sample location is approximately $1 \mathrm{~cm}$ and the inside diameter of the quartz cup $0.7 \mathrm{~cm}$

A picture of a SAM quartz sample cup is shown in Fig. 8. The porous frit, also made out of quartz, is located in the center of the cup. It allows the sample to be centered in the hottest part of the oven and allows helium to flow through the sample and rapidly sweep evolved gas away from the possibly reactive sample and through the manifold for analysis.

\subsection{Gas Processing System}

The Gas Processing System (GPS) enables the gases sampled from the atmosphere or those extracted from solid samples to be manipulated. In addition to bringing this gas to one of the three SAM instruments, gases are separated or enriched in trace species to enable improved sensitivity and accuracy in composition and isotope measurements. The gas processing system consists of the two turbomolecular pumps, the system of valves, manifolds, transfer tubes, heaters, pressure sensors, and temperature sensors. Additionally, it includes the oxygen tank utilized for combustion experiments and a number of gas scrubbers and getters.

Valves, Manifolds, Heaters, and Transfer Tubes: The two high conductance valves and 46 of the 52 micro-valves were designed, fabricated, and tested in-house at the NASA GSFC. The 6 remaining valves were valves manufactured by Aker Industries, Oakland CA. The micro-valves built in-house at the NASA GSFC were electron-beam welded into titanium manifolds as illustrated in Fig. 9. The double latching solenoid micro-valves are designed to operate temperatures of $-40{ }^{\circ} \mathrm{C}$ to $200{ }^{\circ} \mathrm{C}$ while maintaining leak rates in the range of $10^{-9} \mathrm{~atm} \mathrm{cc} / \mathrm{sec}-\mathrm{He}$. A permanent magnet latches the valve in either the open or closed position with the power off and when the solenoid is actuated with a current pulse the valve changes state. The minimum close/open/close time for the valve is 0.02 second, the cycle time of the microsequencer. SAM utilizes 14 custom fabricated manifolds with 1 to 10 valves on each. A highly miniaturized gland assembly utilizing a platinum o-ring served to create an ultra-high-vacuum seal to a $0.032 \mathrm{~cm}$ OD nickel transfer tube that served to interconnect manifolds. Inconel encased heaters from ARI Corporation were wrapped around these transfer tubes and imbedded in groves machined into each manifold. The SAM control circuit was designed to close two of the valves associated with a helium reservoir in the case of sudden power loss so that helium would not be lost while the rover recovered from an unexpected event.

Turbomolecular Pumps: The two SAM electromechanical pumps consisting of a molecular drag pump in series with and spinning on the same shaft as a turbomolecular pump 


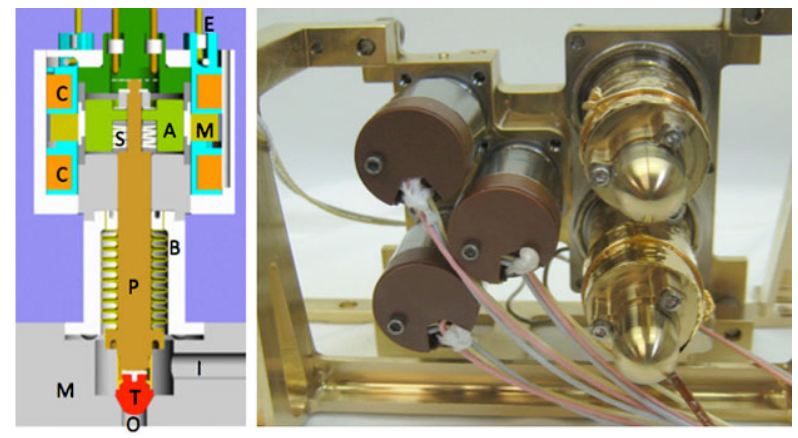

Fig. 9 A schematic of the Goddard designed micro-valve is shown at left with showing $M$ - the samarium cobalt magnets, $A$ - the armature, $B$ - the bellows, $C$ - the coils, $E$ - the electrical feedthrough pins, $P$-the pintle, $T$ - the pintle tip, $M$ - the manifold, and $I$ and $O$ the gas input and output ports respectively. At right manifold \#3 that incorporates the gas calibration reservoir and the oxygen reservoir and valves V34, V35, and V36 is shown. The valve outside diameter is $1.5 \mathrm{~cm}$. Each manifold was thermally isolated from support structure to maintain transfer lines and manifolds at the temperatures required $\left(135^{\circ} \mathrm{C}-200{ }^{\circ} \mathrm{C}\right)$ for efficient transfer of organics during evolved gas experiments

were developed by Creare Inc., Hanover, NH in collaboration with Goddard engineers who lead a study to increase their robustness and lifetime. These pumps spin at 100,000 revolutions per minute and achieve a compression ratio for $\mathrm{CO}_{2}$ of approximately $5 \times 10^{8}$. The molecular drag stage is designed to exhaust directly to the Mars ambient surface pressure of 700 to $960 \mathrm{~Pa}$. Although both pumps (designated WRP1 and WRP2 -Fig. 3) are used for several of the SAM sequences some level of redundancy is provided by the two pumps since the core sequences could be operated in a less efficient manner with only one pump. In the nominal mode of operation WRP1 is dedicated to pumping the QMS and WRP2 serves to evacuate the manifolds and the TLS. The pump controller was designed and qualified at the NASA GSFC.

Hydrocarbon Trap: The primary SAM trap (Fig. 10) utilized for trapping and transferring of organic compounds to the SAM GC is an integral part of MN5 (Fig. 3). Unlike the scrubbers and getters, the HC-trap can be cooled below ambient to more efficiently trap species of interest. It is not only utilized to trap organics extracted from solid samples as these gases are driven through the manifold lines, but also to separate the heavy noble gases $\mathrm{Kr}$ and $\mathrm{Xe}$ from $\mathrm{Ar}, \mathrm{Ne}$, and $\mathrm{He}$ by cooling the high surface adsorbent material in the trap to $-75^{\circ} \mathrm{C}$ or lower. The trapping materials consist of three adsorbents in series; 0.49 gram of $0.38 \mathrm{~mm}$ non-porous silica beads; 0.079 gram of 60/80 mesh Tenax TA; and at the end of the inlet gas stream 0.11 gram of $60 / 80$ Carbosieve $\mathrm{G}$. The volume of each trapping material is contained in a tube approximately $0.64 \mathrm{~cm}$ diameter and $1.1 \mathrm{~cm}$ long. Gas can be directed in either direction through the trap utilizing the 5-stage micro-valve manifold assembly (MN5 in Fig. 3) so when evolved gas from solid samples is being driven through the trap the gas stream first encounters the silica beads, then the more strongly adsorbing Tenax, and finally the Carbosieve. To release these adsorbed gases and transport them to the GC columns via helium carrier gas, the trap is heated and the flow direction is reversed. To minimize power consumption, the three-stage thermoelectric coolers (TEC) which cool the HC-trap are vacuum-insulated from the Mars atmosphere. A hermetic enclosure permanently pumped by a small getter activated at the time of trap assembly provides the required vacuum. Leak rates into the vacuum space are sufficiently low to maintain vacuum over the course of the nominal landed mission. The assembly also incorporates a passive, CTE- 
Fig. 10 Assembly illustration of the HC-trap. The valves are located on MN5

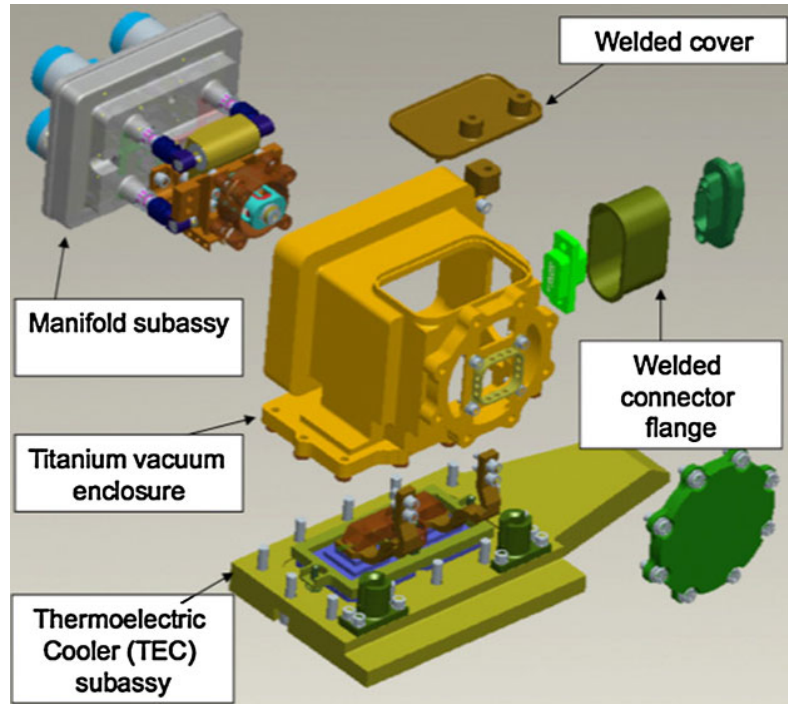

driven thermal switch to protect the TEC's during times when the traps are heated to high temperature $\left(350{ }^{\circ} \mathrm{C}\right)$ to release organic compounds.

Scrubbers and Getters: The scrubber system consisted of 0.64 grams of a zeolite (Linde $13 \times)$ in series with another cell containing a similar volume of magnesium sulfate. The zeolite serves to rapidly remove $\mathrm{CO}_{2}$ from the gas phase to enable a measurement of the residual $\mathrm{N}_{2}$ and its ${ }^{15} \mathrm{~N} /{ }^{14} \mathrm{~N}$ ratio since nitrogen adsorbs much more weakly on the zeolite. The $\mathrm{MgSO}_{4}$ reacts with water to form hydrated sulfates which can subsequently be released on heating. This scrubber serves to trap trace atmospheric water for subsequent thermal release and TLS analysis of its D/H ratio. The getter in the manifold line consists of a SAES ST175 porous mixture of sintered titanium and molybdenum powders that can be reactivated on Mars. This getter serves to remove all active gases from the manifold except $\mathrm{CH}_{4}$ and the noble gases that are not removed by this passive pump. The second getter of a similar design serves to pump active gasses from the QMS at those times when the turbomolecular pumps are not used for this purpose such as during the static mass spectrometer noble gas measurement sequence (AS-NG, Table 6). Activation heater wires were embedded in the getter and radiation shields were designed for placement around the getter to enable it to get to the activation temperature with the available power.

Helium and Calibration Gas Systems. Helium is used throughout the GPS system and serves as the carrier gas for the GC system and to transfers evolved gas to both the QMS and TLS. The Helium storage system consists of redundant manifolds, high-pressure reservoirs, pressure sensors and pressure regulators. Figure 11 shows an exploded view of one of the Helium manifold assemblies. The two-stage regulator on each helium manifold was manufactured by Autoflow Products, Gardena, CA. It was designed to maintain the output pressure constant within several percent as the pressure in each helium tank decreased over the course of the mission from $\sim 140$ to several bar. The pressure in the SAM manifold for GCMS sequences was set by these regulators to approximately 1 bar above the ambient Mars pressure to maintain the optimal conditions for chromatographic separation. The volume in each helium reservoir was $180 \mathrm{~cm}^{3}$ sufficient to utilize the full set of SAM sample cups with a safety factor. Pressure sensors manufactured by Kulite Semiconductor Products, Leonia, N.J. are used to measure the Helium pressure in the reservoirs and on manifolds 
Fig. 11 Assembly drawing of the helium reservoir and two stage regulator assembly

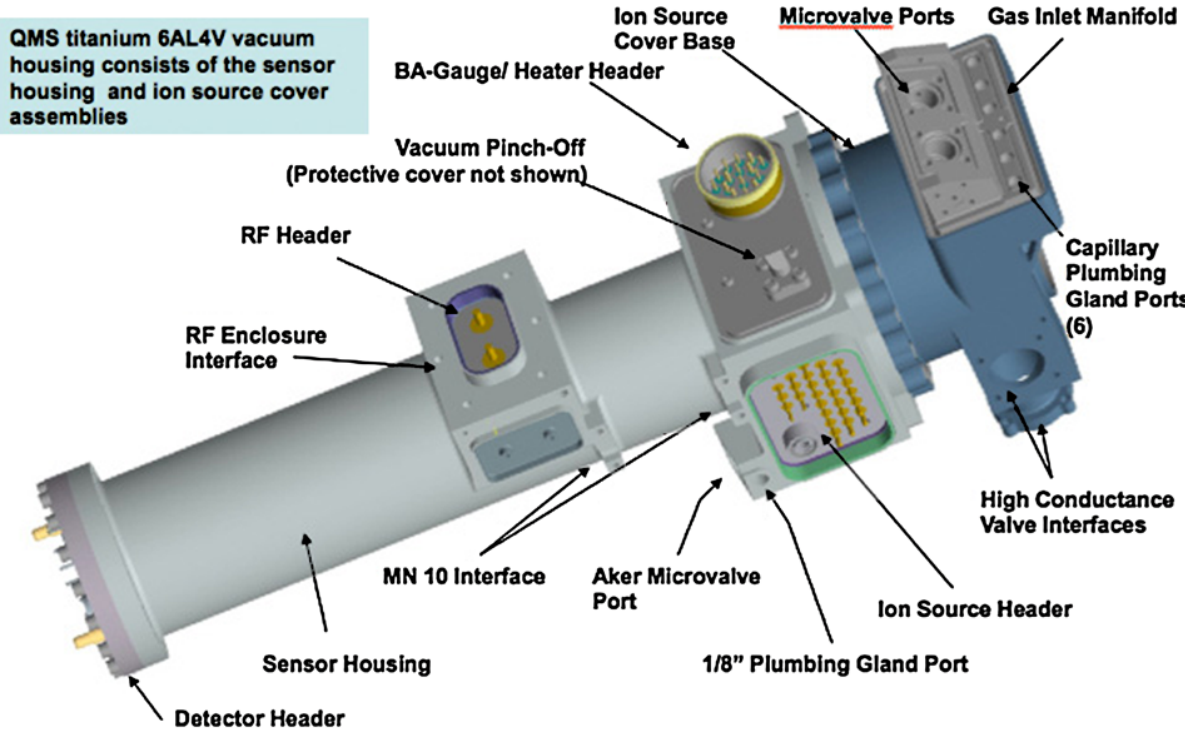

Fig. 12 Model showing various elements of the SAM QMS

MN6 and MN7 (on either side of V20 and V21 valves) to measure the pressure in the GPS. Metal diaphragm sensors are used in the Helium manifolds to prevent Helium permeation across the diaphragm during the long Helium storage time while silicon diaphragm sensors are used in the two other GPS locations. The two low-pressure reservoirs on MN3 (Fig. 3) contain the calibration gas mix and the $\mathrm{O}_{2}$ gas utilized for combustion.

\subsection{Quadrupole Mass Spectrometer}

Mass Analyzer: The QMS (Fig. 12) is derived from high heritage components utilized in previous planetary missions. The mass analyzer is a set of $15.24 \mathrm{~cm}$ long quadrupole rods whose fabrication and rigid assembly is designed to hold their hyperbolic shape over the ion 
path to within $5 \mu \mathrm{m}$. A very similar quadrupole design was utilized for the Galileo Probe Neutral Mass Spectrometer (Niemann et al. 1992). A combination of radio frequency (RF) and static (DC) voltages are applied to opposite rod pairs to achieve mass separation. The voltages $\left(V_{\mathrm{dc}}+V_{\mathrm{ac}} \cos (\omega t)\right)$ and $-\left(V_{\mathrm{dc}}+V_{\mathrm{ac}} \cos (\omega t)\right)$, where $\omega$ is the frequency of $V_{\mathrm{ac}}$, result in a two-dimensional quadrupole field of the form

$$
\varphi(x, y)=\left(V_{\mathrm{dc}}+V_{\mathrm{ac}} \cos (\omega t)\right)\left(x^{2}-y^{2}\right) / R_{0}^{2}
$$

where $R_{0}(0.5 \mathrm{~cm})$ is the distance from the $z$ (symmetry) axis to the nearest rod surface, and $x$ and $y$ are the axes crossing both the $z$ axis and nearest point of the adjacent rods. Three fixed frequencies were used over the 2-535 Da mass range of QMS: $3.013 \mathrm{MHz}$ for the mass range 1.5 to $19.5 \mathrm{Da}, 1.438 \mathrm{MHz}$ for the range 19.5 to $150.5 \mathrm{Da}$, and 0.853 for the range 150.5 to $535.5 \mathrm{Da}$. Small amplitude changes in the RF are made under software control to compensate for temperature and frequency drifts. These corrections keep the analyzer tuning essentially constant over the instrument operating temperature range of $-40{ }^{\circ} \mathrm{C}$ to $+50{ }^{\circ} \mathrm{C}$.

Mass Scan Modes: The SAM flight software is designed to allow the QMS detectors to sample during any $0.017 \mathrm{sec}$ integration period (IP) any unit or fractional $\mathrm{m} / \mathrm{z}$ value within the 1.5 to $535.5 \mathrm{Da}$ mass range of the instrument with a resolution of $0.1 \mathrm{Da} .0 .003$ seconds of deadtime elapse before counts are summed in a different $m / z$ value. In addition, the $\mathrm{RF} / \mathrm{DC}$ rod voltages can be independently and precisely set to transmit a selected range (band) of $m / z$ values in a single IP. A variety of mass scan modes are utilized to optimize return from any particular measurement. A unit mass scan designates a mass scan over a selected range of $\mathrm{m} / \mathrm{z}$ values stepping to the nominal unit mass value. A high-resolution mass scan is most often implemented with a 0.1 Da step size. A combination of these two modes combined with sampling at both $20 \mu \mathrm{A}$ and $200 \mu \mathrm{A}$ emission has proven useful for measurements where the gas density in the ion source is constant or changing slowly with time as in the atmospheric measurements. When more rapid sampling of transients is needed as in the case of GCMS experiments where the GC peak may be only a few seconds wide, the bands are utilized extensively. A combination of the band data and the GC Thermal Conductivity Detector (TCD) data allows the peak shape to be determined and allow correction for a single $\mathrm{m} / \mathrm{z}$ signal even when this mass value is substantially under sampled.

Ion Source: The SAM ion source is adapted from that developed for the Comet Nucleus Tour (CONTOUR) mass spectrometer and utilized a W/3 \%Re wire as its electron source. The electron emission level is set by a script parameter and typically emission currents $20 \mu \mathrm{A}$ or $200 \mu \mathrm{A}$ are utilized. More than an order of magnitude increase in sensitivity from the Cassini Ion and Neutral Mass Spectrometer (Kasprzak et al. 1996) was realized in the CONTOUR instrument with optimization of the electron and ion optics design and the SAM mass spectrometer demonstrates similar sensitivity $\left(\sim 5 \times 10^{-3}\right.$ (counts/sec)/( $\mathrm{N}_{2}$ particle/cc).

Detector: The SAM detector assembly incorporated redundant continuous dynode secondary electron multipliers. A gain of more than 50 million was typical for these model 4870 detectors mounted in a housing also adapted from the CONTOUR mass spectrometer design. The detectors were utilized in a pulse counting mode and the dark current of each detector was less than one count/minute.

\subsection{Gas Chromatograph}

The gas chromatograph assembly (Fig. 13) contains six complementary chromatographic columns (Table 9) each $30 \mathrm{~m}$ in length with an internal diameter of $0.25 \mathrm{~mm}$. The stationary phases of the columns are selected to provide a broad range of detection capability for 
Fig. 13 A picture of the SAM gas chromatograph assembly prior to integration into the suite

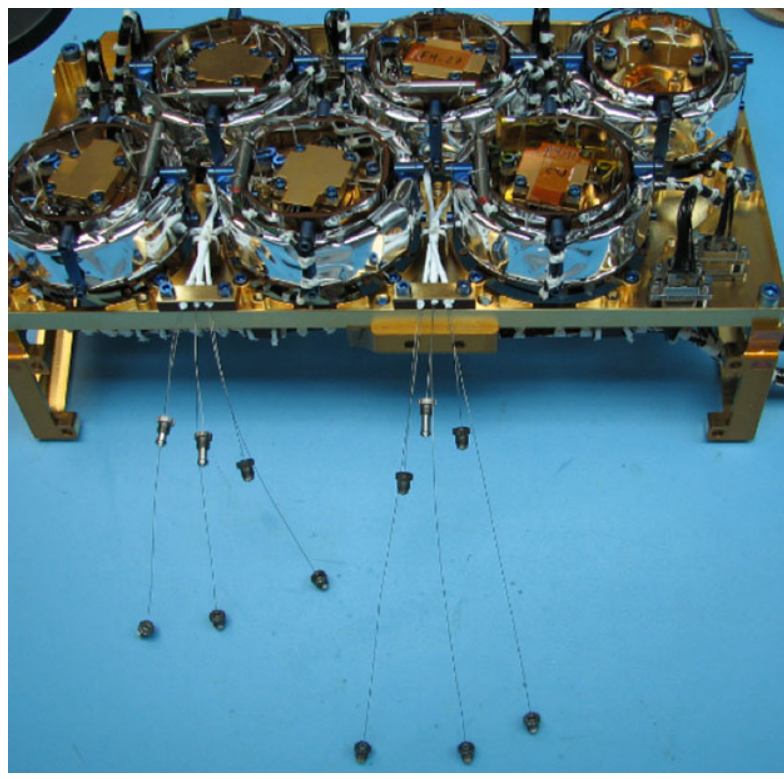

Table 9 Gas chromatograph columns

\begin{tabular}{|c|c|c|}
\hline Column & Stationary phase & Species targeted \\
\hline GC1-MXT 20 WCOT & $\begin{array}{l}\text { Polydimethylsiloxane with } \\
20 \% \text { of phenyl }\end{array}$ & $\begin{array}{l}\text { Medium molecular weight organics } \\
\text { (C5-C15 organics) }\end{array}$ \\
\hline GC2-MTX 5 (WCOT) & $\begin{array}{l}\text { Polydimethylsiloxane with } \\
5 \% \text { of phenyl }\end{array}$ & $\begin{array}{l}\text { High molecular weight VOCs including } \\
>\text { C } 15 \text { chemical derivatives }\end{array}$ \\
\hline GC3-Carbobond (PLOT) & Carbon molecular sieve & Permanent gases and $\mathrm{C} 1-\mathrm{C} 2 \mathrm{HCs}$ \\
\hline GC4-Chirasil- $\beta$ Dex CB & $\beta$ cyclodextrin & Enantiomers of VOCs \\
\hline GC5-MXT CLP (WCOT) & $\begin{array}{l}\text { Polydimethylsiloxane with } \\
\text { phenyl and cyanopropyle }\end{array}$ & $\begin{array}{l}\text { Medium molecular weight organics } \\
\text { (C5-C15 organics) }\end{array}$ \\
\hline GC6-MXT Q (PLOT) & $\begin{array}{l}\text { Divinylbenzene or } \\
\text { substituted divinylbenzene }\end{array}$ & $\begin{array}{l}\mathrm{C} 1-\mathrm{C} 4 \text { VOCs } \mathrm{NH}_{3}, \mathrm{~S} \text { containing } \\
\text { compounds }\end{array}$ \\
\hline
\end{tabular}

PLOT $=$ porous layer open tubes; WCOT $=$ wall coated open tublar; VOC $=$ volatile organic compounds

both light and heavy organic molecules for a range of molecular polarity and for inorganic volatiles. Nevertheless, there is significant overlap in several of the columns such as GC1, GC2, and GC4 each of which can transmit a range of mid-molecular weight compounds (Table 9). Three of the columns (GC4, GC5, and GC6) incorporate a small adsorption trap in line with the column, using either Tenax TA for organics, or Carbosieve SIII for inorganic volatiles or the lightest organics, as the adsorbent. The role of these traps is to preconcentrate the analytes, and then to release them as fast as possible in the GC columns by flash heating to increase the separation power of the chromatograph. The analytes are released from the injection trap by a typical 4-10 seconds actuation of a heater that provides local heat to the trap. Very heavy organic compounds might not transmit through the injection trap and the injection mode for GC1 and GC2 is to trap these heavy organic compounds on the entrance to the GC column prior to initiation of the column heating ramp. GC3 is specifically 
included to provide the option of analyzing permanent gases and very light hydrocarbons. Each column is independently heated by the proportional integral differential (PID) heater circuit provided by the SAM electronics. Only one column is operated at a time. Five of the columns incorporate a TCD that provides a signal independent of the mass spectrometer. This detector can detect the major species contained in the sample down to the part per million level, with a $10^{5}$ dynamic linear range. This detector, which generates a simple physical signal, not only provides an additional level of redundancy, but also the peak shapes derived from the TCDs can be utilized to better fit the GCMS data from the QMS where there is the possibility of under sampling of a fast eluting GC peak.

\subsection{Tunable Laser Spectrometer}

Unlike the QMS, SAM's tunable laser spectrometer (TLS) has no survey ability, but rather targets specific gases $\left(\mathrm{CH}_{4}, \mathrm{CO}_{2}\right.$, and $\left.\mathrm{H}_{2} \mathrm{O}\right)$ and high-precision ratios of their isotopic species in $\mathrm{H}, \mathrm{C}$, and $\mathrm{O}$. TLS uses a technique long established in terrestrial applicationsthat of long-pathlength infrared laser absorption within a multipass sample cell to record ultra-high resolution $\left(0.0005 \mathrm{~cm}^{-1}\right)$ spectra of selected lines of targeted species. The method is a direct, non-invasive, simple technique that can produce remarkable sensitivities (sub parts-per-billion) for gas detection and isotope ratio determinations (Webster 2005). By choosing spectral line regions either predicted by the High Resolution Transmission Molecular Absorption Database (HITRAN) line list or studied empirically with gas mixtures, tunable laser spectrometers can avoid the mass interferences of mass spectrometers (e.g. CO and $\mathrm{N}_{2}, \mathrm{~N}_{2} \mathrm{O}$ and $\mathrm{CO}_{2}, \mathrm{CH}_{3} \mathrm{D}$ and ${ }^{13} \mathrm{CH}_{4}$, HDO and $\mathrm{H}_{2}^{17} \mathrm{O},{ }^{13} \mathrm{CO}_{2}$ and ${ }^{17} \mathrm{OCO}$ ), or the need to convert gases like $\mathrm{CH}_{4}$ to $\mathrm{CO}_{2}$ for isotopic analysis. In this way, TLS complements the measurements of the MS, and focuses on specific measurements for which it offers increased precision. Of course, because infrared spectroscopy relies on changes in molecular dipole moments with vibration, TLS has no capability for noble gas measurements.

Two semiconductor continuous-wave laser sources scan over three wavelength regions chosen to target gas abundances and isotope ratios: a near-infrared (NIR) tunable diode laser at $2.78 \mu \mathrm{m}$ for carbon dioxide and water, and an interband-cascade (IC) laser at $3.27 \mu \mathrm{m}$ for methane. The NIR laser from Nanoplus, Germany operates at room temperature with a single stage thermoelectric cooler (TEC), and the JPL-built IC laser operates at $245 \mathrm{~K}$ with a two-stage TEC. Detectors are high-sensitivity $\mathrm{HgCdZnTe}$ photovoltaics with immersion lenses provided by Vigo Systems S.A. of Poland.

TLS scans over selected rotational lines within a given vibrational band. At typical Mars pressures near 7 mbar, the molecular line shapes associated with the rovibrational lines of interest are close to Doppler-limited. So narrow is the laser linewidth compared to the molecular linewidth, that in the convoluted spectrum, the individual lines show insignificant loss in their absorption optical depth and are well-resolved. Target spectral regions are chosen for strong lines but with minimal spectroscopic interference, and comparable temperature dependence important for isotope ratio determinations. Combined with a relatively long pathlength (an 81-pass Herriott cell with mirror separation near $20 \mathrm{~cm}$ ) the high spectral resolution results in high-sensitivity detection for the stand-alone TLS with limits at the parts-per-billion level.

SAM leverages the capabilities of TLS with the ability of the suite to enrich target gases or trap out unwanted gases, so that significant increases in gas sensitivity may be achieved. For TLS, methane enrichment is one example. Another example is the scrubbing of carbon dioxide to reduce interferences in the water spectral region. This latter gas processing provides mitigation to the earlier descoping of a third TLS channel for water isotopes at 


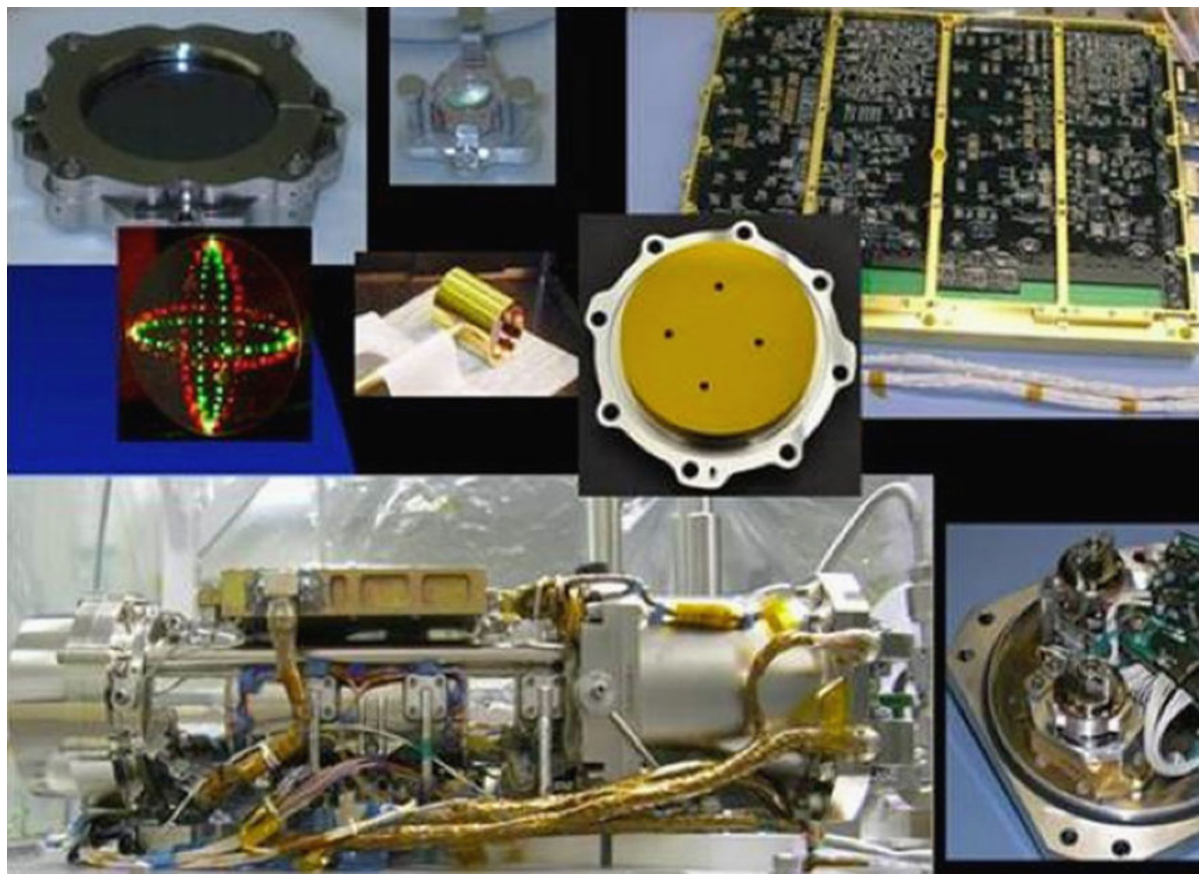

Fig. 14 Photographs of components of the TLS flight hardware. Left to right across three rows: Ge wedge window assembly, detector field lens mount, main electronics board, alignment laser pattern for two channels on one mirror, reference gas cell, far mirror of Herriott cell, complete TLS spectrometer, and the laser plate prior to installation

$2.68 \mu \mathrm{m}$ and the replanning to make these measurements in the carbon dioxide region near $2.78 \mu \mathrm{m}$.

The TLS spectrometer design (Fig. 14) is based on a central multipass Herriott cell defined by two identical spherical mirrors set $20 \mathrm{~cm}$ apart. The mirrors are aluminum with gold-nickel coatings, and drilled with small holes $(2.5 \mathrm{~mm}$ diameter $)$ that allow the laser beams to enter and exit. The main sample gas volume is defined by an internal diameter of $\sim 5 \mathrm{~cm}$ and a length of $\sim 20 \mathrm{~cm}$ producing a cell volume of $\sim 405 \mathrm{~cm}^{3}$. This "Herriott" cell is fitted with 4 high vacuum (Aker) valves that connect to the SAM gas handling system under its control, allowing evacuation and filling of the Herriott cell directly from the atmosphere or from the SAM gas handling system. The cell is fitted with two heaters for fringe washing, its own pressure gauge and temperature sensors. The TLS Herriott cell is designed (Tarsitano and Webster 2007) to provide 81 passes $(16.8 \mathrm{~m})$ for methane measurements, and 43 passes $(8.93 \mathrm{~m})$ for carbon dioxide and water measurements. Although the Herriott configuration provides its greatest stability by having the laser beam enter and exit the same hole, this is barely compromised in the TLS configuration of exiting at the far mirror, with the advantage of reducing the packing density that would be needed in the foreoptics chamber to accommodate the additional detectors and lenses.

Two additional chambers are joined to this central one through wedged Ge windows and o-ring seals - a foreoptics chamber at one end, and a small science detector chamber at the other end. The foreoptics chamber houses the lasers and their collimators, reference detectors, reference gas cells, and beamsplitters that therefore sit outside the main. The foreoptics chamber has its own pressure gauge and internal temperature sensors. 
Table 10 TLS measurement capability

\begin{tabular}{|c|c|c|c|}
\hline Channel & Wavelength & Scan name & 15 minute predicted capability \\
\hline 1-IC laser & $3.3 \mu \mathrm{m}$ & Methane & $\begin{array}{l}\text { to } 0.3 \text { ppbv } \\
\delta^{13} \mathrm{C} \text { to } 2 \text { per mil }\end{array}$ \\
\hline \multirow[t]{2}{*}{ 2-Near IR laser } & $2.785 \mu \mathrm{m}$ & Carbon dioxide & $\begin{array}{l}\text { to } 0.2 \text { ppmv in } \mathrm{CO}_{2} \text { and } \mathrm{H}_{2} \mathrm{O} \\
\delta^{13} \mathrm{C} \text { to } 2 \text { per mil } \\
\delta^{18} \mathrm{O} \text { to } 3 \text { per mil } \\
\delta^{17} \mathrm{O} \text { to } 5 \text { per mil }\end{array}$ \\
\hline & $2.783 \mu \mathrm{m}$ & Water & $\begin{array}{l}\mathrm{H}_{2} \mathrm{O} \text { to } 0.1 \text { ppmv } \\
\delta \mathrm{D} \text { to } 2 \text { per mil } \\
\delta^{18} \mathrm{O} \text { to } 3 \text { per mil } \\
\delta^{17} \mathrm{O} \text { to } 5 \text { per mil }\end{array}$ \\
\hline
\end{tabular}

Spectral regions for TLS were carefully selected for high sensitivity in detection but insensitivity to temperature in line pairs chosen for isotope ratio measurements. TLS employs both direct absorption and second harmonic (2f) detection techniques that enhance its sensitivity since the $2 \mathrm{f}$ signal is zero-based and the detection regime is moved to higher frequencies $(\sim 4 \mathrm{kHz})$ where $1 / \mathrm{f}$ noise is lower and bandpass techniques can be used.

TLS Water and Carbon Dioxide Measurements: TLS will measure the ${ }^{13} \mathrm{C} /{ }^{12} \mathrm{C}$ and $\mathrm{D} / \mathrm{H}$ isotope ratios in methane; the ${ }^{13} \mathrm{C} /{ }^{12} \mathrm{C}$ and ${ }^{16} \mathrm{O} /{ }^{17} \mathrm{O} /{ }^{18} \mathrm{O}$ isotope ratios in $\mathrm{CO}_{2}$; and the $\mathrm{D} / \mathrm{H}$ and ${ }^{16} \mathrm{O} /{ }^{17} \mathrm{O} /{ }^{18} \mathrm{O}$ isotope ratios in water. While formal instrument requirements specify isotope ratio capability to 10 per mil $(0.1 \%)$, flight instrument environmental testing shows that in some cases we can expect to exceed these requirements and determine specific isotope ratios to a few per mil.

The predicted sensitivities for the TLS target gases and isotope ratios are given in Table 10 . For carbon dioxide and water abundances, TLS has very high sensitivity that will be valuable in the atmospheric, pyrolysis, and combustion experiments.

The TEC temperature settings of the NIR $2.78 \mu \mathrm{m}$ laser can be changed to sequentially access two regions: one for carbon dioxide at $2.785 \mu \mathrm{m}$ and the other for water at $2.783 \mu \mathrm{m}$. Although the water region is not ideal for water isotope measurements in that it has some interference from carbon dioxide, it will be very valuable for water isotope determinations of hydrated minerals, since the EGA thermal processing is expected to produce large water amounts. Enrichment utilizing the SAM water scrubber followed by its release after other gases have been removed from the manifold could also reduce interferences. Water abundances can be measured in the cell using both of these channels to provide a cross-check.

Methane and Its Isotopes: Fig. 15 shows a comparison between isotope ratio results for a laboratory tunable laser spectrometer prototype of TLS and the flight instrument as compared with a commercial IRMS mass spectrometer tailored for methane isotope ratio determination. The agreement of the fit was excellent-at the 2-5 per mil level, with scatter of a few per mil, showing the capability for methane isotope ratio measurement on Mars. However, this data is for methane abundances much higher than expected and is not using the flight TLS instrument itself. Flight data calibration results are summarized in Sect. 8.9.

Methane Enrichment: TLS methane capability is enhanced within the SAM suite by several factors. First, SAM provides the capability to repeatedly pump out the TLS cell, and seal it with a full sample of atmosphere that allows very long integration times (e.g. during the night) that are possible with such a stable, unreactive gas. Second, SAM provides crosschecking of related measurement data and housekeeping that provide additional context. Most directly, SAM provides methane enrichment through the Chemical Separation 
Fig. 15 Methane ${ }^{13} \mathrm{CH}_{4}$ isotope measurements. Top: Spectral scan from flight instrument with $x$-axis in wave numbers. Middle: isotope ratio measurement data from 2 minute averages with 4 ppmv methane that with a SAM enrichment of 100 would be equivalent to sampling $40 \mathrm{ppbv}$ on Mars. The sample is enriched four-fold over terrestrial. Bottom: Isotope data (points) and its mean value (dash) from a TLS prototype compared to IRMS mean value (solid line) as recorded by Lance Christensen, JPL

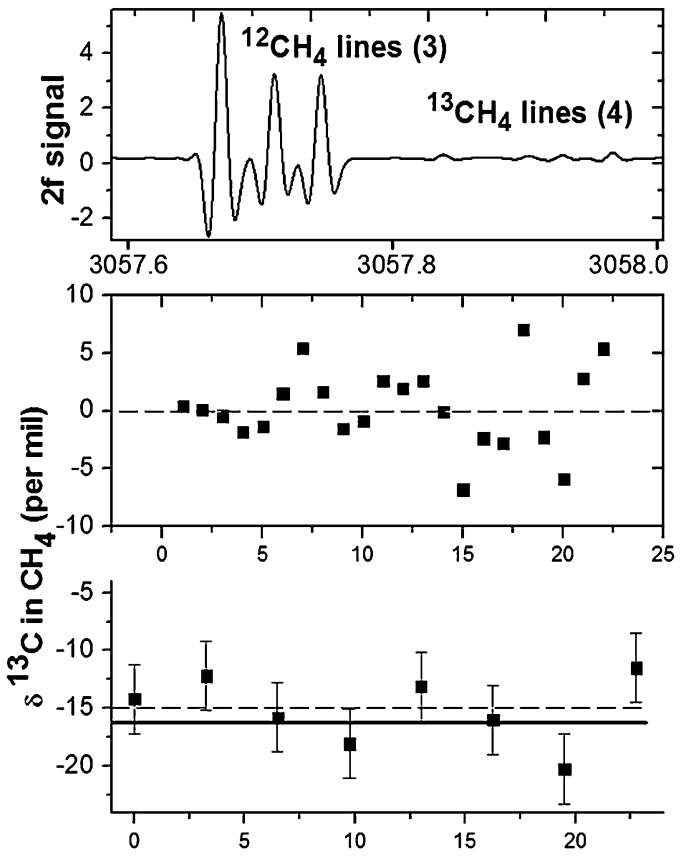

and Processing Laboratory (CSPL), in particular the ability to flow and trap gas over time, remove atmospheric $\mathrm{CO}_{2}$ through a heated chemical scrubber, and enrich in methane with these and with a hydrocarbon trap (Sect. 2.4).

Two basic processes are employed that use a getter and a hydrocarbon trap. First, a titanium/molybdenum based getter is used that operates at $25^{\circ} \mathrm{C}$ but is baked out for use at $800{ }^{\circ} \mathrm{C}$, and this absorbs active gases except noble gases (Ar is $1.6 \%$ abundance in Mars atmosphere) and $\mathrm{CH}_{4}$ to produce a methane enrichment of $\sim 50$. To increase this factor, the SAM HC-trap is utilized to increase the $\mathrm{CH}_{4}$ density in the TLS. Methane enrichment by a factor of 50-100 is expected in routine operations with SAM. Studies on the laboratory SAM testbed are planned to understand the resource requirements for further enrichment prior to surface operations.

Methane abundance and its D/H Ratio: The spectral region chosen for the TLS methane measurements is shown in Fig. 15, accessing a group of three strong lines of ${ }^{12} \mathrm{CH}_{4}$, four strong ${ }^{13} \mathrm{CH}_{4}$ lines, and a single strong $\mathrm{CH}_{3} \mathrm{D}$ line adjacent to the strongest ${ }^{12} \mathrm{CH}_{4}$ line. While all the isotopic lines are inherently strong absorbers (similar line strengths), the lower abundances of the isotopic forms means that the ${ }^{13} \mathrm{CH}_{4}$ lines are $\sim 1 / 80$ th that of the ${ }^{12} \mathrm{CH}_{4}$ lines, and the $\mathrm{CH}_{3} \mathrm{D}$ line $\sim 1 / 50$ th of the ${ }^{13} \mathrm{CH}_{4}$ lines, despite the higher D/H on Mars $(\times 5)$ compared to Earth.

\subsection{Electronics Architecture}

Rover Interface: 5 independent, Rover switched, power feeds with a nominal voltage of $28 \mathrm{~V}$ (voltage range is $24 \mathrm{~V}$ to $33 \mathrm{~V}$ using a JPL standard chassis-referenced bipolar bus architecture). Bus 1 and 2 supply pre-regulated DC/DC converters that exhibit a constant power characteristic. Busses 3, 4, and 5 provide power to switched resistive heater loads.

Any, or all, buses may be turned on in any order, however the normal mode of operation is to have all busses operating with the minimum power in the range of approximately $25 \mathrm{~W}$ 
(all on Bus 1) and the maximum possible power from all busses is approximately $990 \mathrm{~W}$. Power usage during experiment operation is a function of the commanded instrument mode with the power sequencing on $30 \mathrm{~ms}$ intervals.

Bus 1 provides power to the instrument control functions, mass spectrometer system and wide range pump system while Bus 2 provides power to the TLS, GC, pyrolysis subsystem and regulated heater system. Both busses incorporate a power limiter function that can actively reset that is reset the instrument via an automatic power on/off cycle. The loads on Busses 3, 4 and 5 do not have a limiter.

The power interface also includes 4 Platinum Resistive Temperature (PRT) temperatures monitored by the Rover system. The Data Interface consists of two, high speed ( $2 \mathrm{Mb} / \mathrm{s})$, serial busses using $5 \mathrm{~V}$ logic levels balanced relative to system ground. Primary and redundant discrete command lines are used for bus selection with only one bus is active at a time.

SAM Module Overview: Most of the SAM instrument electronics are integrated into the $M E B$ as 8 slices or modules. The list below is in the order they are incorporated as slices into the MEB.

- $V H$-Valve/Heater

- PS-Power Supply

- $P T$-Pyrolysis/Thermistor

- $F B$-Filament/Bias

- $\mathrm{CDH}$-Command Data Handling

- MOT-Motor Control

- GCE-Gas Chromatograph Electronics

- TLSE-Tuned Laser Spectrometer Electronics

The three stand-alone modules are the $R F$ (Radio Frequency) which is attached to the Quadrupole Analyzer housing, the $H V$ (High Voltage) and the PDB (Power Distribution Box). With the exception of the PDB, all of the modules receive their power from the common SAM Suite power supply.

Power Supply (PS) Module: The PS incorporates four regulated converters into a single module with two converters (MAIN and MOTOR) sharing Bus 1, and two converters (AUX1 and AUX2) sharing Bus 2. All four converters are slaved to the MAIN power supply $50 \mathrm{kHz}$ oscillator providing a total of 25 different $\mathrm{AC}$ and DC voltages associated with 9 separate ground systems. An EMI filter and soft start are used for each of the two power inputs together with the power limiter described above. Current monitors are provided for each of the 4 power supplies. There are no current monitors for the resistive heater loads powered through the PDB. The PS is not a redundant system but is designed be very reliable through the combination of robust derating and incorporation of protective circuitry.

Command and Data Handling (CDH) Module: The CDH (Command and Data Handling) module (Fig. 16) includes a number of functions. The CPU is the Coldfire CF5208 running at $20 \mathrm{MHz}$. The $\mathrm{CDH}$ communicates with the Rover via redundant $2 \mathrm{Mbps}$ high speed RS422 serial bus along with a discrete interface (NMI). The $\mathrm{CDH}$ also interfaces with the VH, MOT, GC, and TLS modules via a RS-422 interface. The boot software resides in a $32 \mathrm{k}$ word PROM. There are 2 Mbytes of RAM, and 1 Mbyte of EEPROM. Flight Software including scripts and tables, are uploaded into the EEPROM as required.

A master AMUX (16), with 4 A/Ds (2 for Housekeeping and 2 for PWMs, is used to switch additional module AMUXs (6), for a total of 256 channels of Analog data. There are 16 8-bit DACs (4 are spares) and 4 16-bit DACs.

Valve/Heater ( $\mathrm{VH}$ ) Module: The VH module provides a $7 \mathrm{amp}$ forward or reverse drive pulse to any one of the 52 micro valves as well as regulated switched power to 60 heaters ( 9 


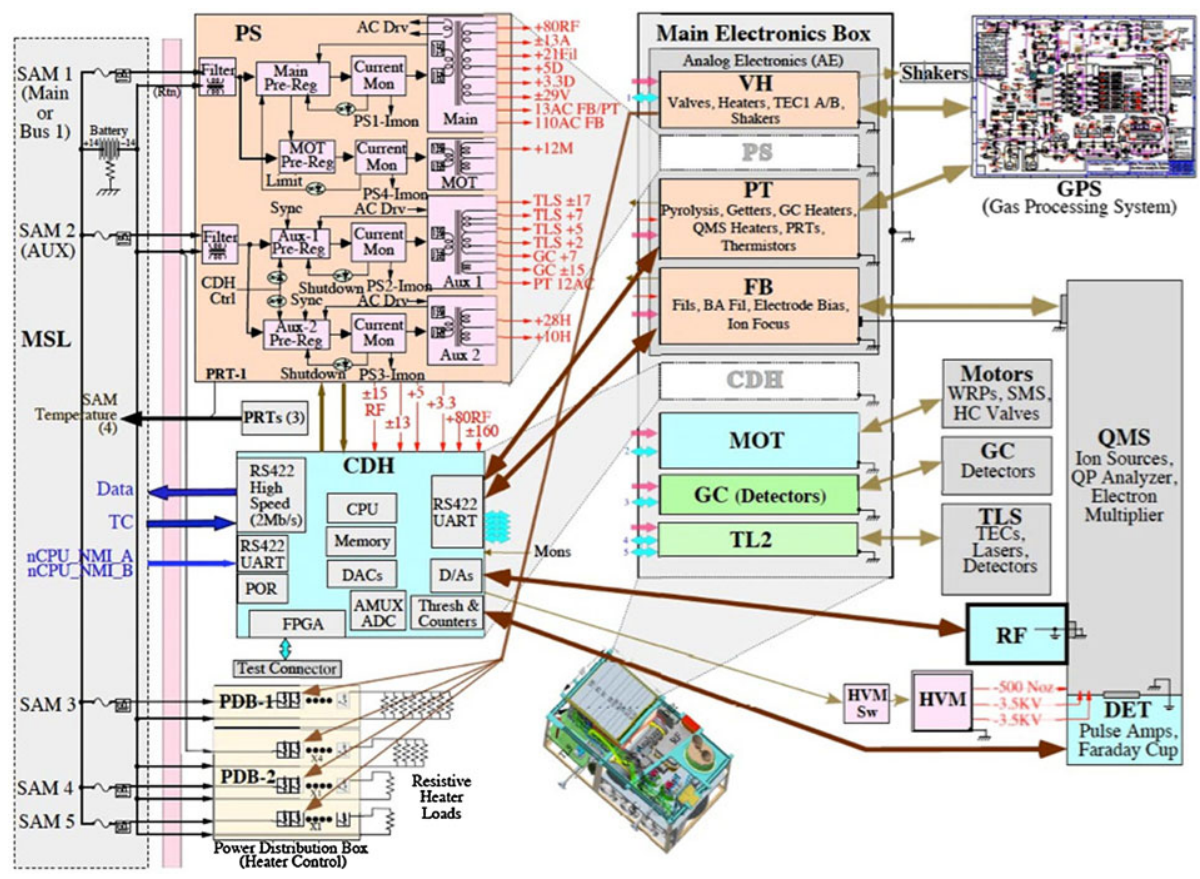

Fig. 16 SAM electrical block diagram showing the eight elements of the Main Electronics Box (MEB), the power distribution modules, the RF and detector modules and the interfaces to the rover, the instruments, the sample manipulation system, and the gas processing system

at $28 \mathrm{~V}$ and 51 at $10 \mathrm{~V}$ ) under the control of the $\mathrm{CDH}$. It also generates the $\mathrm{AC}$ drive sweep for the SSIT shakers. The original VH design included 60 regulated heaters $(9$ at $28 \mathrm{~V}$ plus 51 at $10 \mathrm{~V})$.

Energy storage for the valve driver is provided by an $1100 \mu \mathrm{f}$ capacitor bank with a typical pulse of 7 amps for 0.003 seconds. The drive is bipolar with dual capacitor banks for energy storage at both +30 volts and -30 volts. The separation of the plus and minus pulses can be as short as 0.03 seconds. The recharge rate for the energy storage system restricts the spacing of same-polarity pulses to at least 0.5 seconds.

Pyrolysis/Thermistor (PT) Module: The PT module provides dual programmable 0 to 10 volt precision DC at up to 4 amps for the pyrolysis function and other programmable heaters. Each of the drivers uses a dedicated magnetic amplifier for PWM control of output with better than 14-bit accuracy (over the range of 1 to 9 volts). Each output can be switched to one of six outputs such that the system service 12 individual precision heater functions. Both of the drivers can be operated simultaneously with the capability of providing up to 35 watts on an individual output or 45 watts when the outputs are operated together. The pyrolysis output is functionally enabled on the first switch for each of the outputs and incorporates a synchronous precision current source and associated sampling circuit to allow a direct feedback 16-bit measurement of the associated sample cup temperature.

In addition to the precision heaters and pyrolysis functions, the PT module also services multiple analog functions including 4 pressure sensors and 63 temperature sensors using multiplexed excitation and sampling. The temperature sensors consist of a mix of $10 \mathrm{k} \Omega$ and $30 \mathrm{k} \Omega$ YSI thermistors and $500 \Omega$ Platinum Resistive Temperature (PRT) sensors. 
Filament/Bias (FB) Module: The FB module provides the filament power and control plus the many electrode bias voltages required to operate the QMS Ion Source (IS). 35 regulated voltages, ranging from $-200 \mathrm{~V}$ to $+160 \mathrm{~V}$ to the QMS electrodes in order properly focus the electrons and ions. Each of the electrode outputs are monitored to detect an electrode shorted to ground.

The filament power (in the range of 2 volts and 2 amps) for each of the two IS filaments (which cannot operate simultaneously) is provided by a dual-loop controller to allow slow start-up of the filament in a voltage controlled mode followed by transition to emission control. The emission control level is programmable from $10 \mathrm{uA}$ to $400 \mathrm{uA}$ with control normally set to either 20 to $200 \mathrm{uA}$. A unique feature of the emission control loop design is that the filament floats at $-70 \mathrm{~V}$ using a virtual ground configuration in order to directly measure the emission current.

The Bayard Alpert (BA) gauge filament is controlled by a single voltage loop under DAC control and does not have direct emission regulation. The BA gauge is normally be used to check the internal sensor pressure before turning on the QMS filament but can be run simultaneously with the QMS as a continuous pressure monitor. As part of the BA measurement circuit, the filament is floated at +20 volts using a virtual ground configuration to allow a direct emission measurement. +160 volts for the Collector and an electrometer capability are also provided in order to make the pressure measurement.

Motor Controller (MOT) Module: The MOT module operates and monitors the 6 motors located in the SAM Instrument, as well as several monitors associated with the sample cup loading and operation in the Sample Manipulation System (SMS). The two wide range pumps operate at 100,000 RPM and utilize 3 phase windings and the back EMF to sense the phase for the winding commutation. A multi-stage control sequence is needed for WRP startup to bring the pump up to speed as rapidly as possible without exceeding the trip current of the power supply. Two motors controlled by the MOT open and close high conductance vacuum valves in the GPS (Gas Processing System). These motors use a Hall sensor for commutating. The motor current is used to set and sense the force in the valve-closed position.

An additional MOT-controlled motor used to rotate the sample carousel, and another one used to elevate the selected sample cup into one of the SAM ovens. There are several optical sensors associated with the carousel and elevator whose electrical circuits reside on the MOT module. Three Actel FPGA devices are used to control the many functions of this module and several A/Ds and AMUXs to monitor these operations. The MOT communicates with the $\mathrm{CDH}$ board via a 422 interface. The MOT controller utilizes a state machine implementation.

Gas Chromatograph Electronics (GCE) Module: The GCE module resides in the MEB and serves to control and monitor the Thermal Conductivity Detector (TCD) sensors. The GC column subsystem includes a number of heaters and PRTs that are controlled by the PT module. An Actel FPGA is used as a controller for the GC module functions. The GC module communicates with the $\mathrm{CDH}$ board via a 422 interface.

TLS Electronics and Flight Software: TLS on SAM is designed to be a two-channel laser spectrometer built up on a single board (Fig. 14) and controlled by experimental sequence scripts run from the SAM CDH. Each of the two channels has its own independent and nearly identical electronics, differing only in the type of laser technology they support. Each channel has its own rad-hard Aeroflex 8051 microcontroller for overall process control, communications/error handling, laser/TEC control and data acquisition. Since the instrument is fully controlled by SAM, TLS FSW implements a command/response type of communications protocol that, with the exception of continuous laser health monitoring, never initiates any actions on its own but is instead completely commanded by SAM sequencing. 
FSW process control initializes the instrument and then enters a quiescent state where it waits for commands to arrive in a packet from the RS-422 interface. As a command arrives, it is parsed and if it is found to be free of errors, the command is executed and the software returns to the quiescent state. Laser and TEC software control is performed through a Pulse Width Modulation Digital to Analog Converter (PWM-DAC) scheme that filters a rapidly switching digital signal to a DC level. These filtered DC signals are then presented to analog drive circuitry for their respective functions. Data is acquired through a 16-bit ADC that multiplexes over 6 different spectral signals and 10 other housekeeping readings of pressures, temperatures and voltages. The pressure sensor and thermistors are the only shared resources between the channels and either channel may read them at any time. These devices and their signals are handled by the preamp board, which the channels also share common real estate to provide high gain amplification of the initially weak (micro-amp) detector signals.

Laser light is channeled via steering optics and a beam splitter through both a reference cell and the main sample cell, resulting in two detectors per laser channel. In addition to producing a simple trapezoidal laser-tuning ramp, the drive circuitry also adds a small sine wave modulation on top of the ramp to implement a Wavelength Modulated Spectroscopy (WMS) technique. The resulting detector signal is demodulated at twice the original frequency, yielding a second harmonic, or $2 \mathrm{f}$ spectra. This design produces three signals per detector: an un-modulated direct absorption spectra (synchronously sampled to remove modulation effects), a low gain $2 \mathrm{f}$ spectra and a high gain $2 \mathrm{f}$ spectra. Thus, there are six different scans resulting from each laser: three from the measurement Herriott cell (direct absorption, $2 \mathrm{f}$ low-gain, $2 \mathrm{f}$ high-gain) and three from the reference channel that contains calibration gases within tiny optical reference cells housed in the fore-optics and sampled by the same laser simultaneously using a beam-splitter. Finally, a simple switch was added to allow the NIR laser channel to switch between non-adjacent scanning windows to accommodate measurements of $\mathrm{H}_{2} \mathrm{O}$ and its isotopologues.

Radio Frequency Electronics Circuit $(R F)$ : The amplitude of the DC and RF voltages provided to the QMS rods is controlled by the analog output of 16 bit DACs located in the $\mathrm{CDH}$. The RF tuning requires an unusually high accuracy and stability. For example, a 1/10th Da shift can result from an amplitude change of $0.03 \%$ or a frequency change of $0.015 \%$. This sensitivity requires tight requirements for the stability of these open loop analog circuits over the wide temperatures that this circuit is required to operate under. A circuit $\mathrm{Q}$ of about 300 is required to achieve low power $(8 \mathrm{~W})$ and high voltage $(1500 \mathrm{~V}$ p-p).

Power Distribution Box (PDB): The PDB module has switches to control 14 resistive heaters operating directly off of Busses 3-5. To achieve the load isolation required by the Rover, a solid-state (optical) relay is used between the control signal from the VH module and the loads. Figure 16 illustrates the general distribution of the power.

High Voltage Module (HVM): The HVM is a stand-alone module that uses three separate oscillators to generate 0 to $-3.5 \mathrm{kV}$ under DAC control for each of the two electron multipliers plus a switched $-500 \mathrm{~V}$ output for the QMS nozzle. In order to protect against Paschen breakdown internal to the QMS, the HVM powers up in the 0 voltage state. Special Paschen breakdown immune cables and connectors are utilized for the outputs and the connections to the QMS.

Detector (DET): The DET receives, amplifies, discriminates, and counts pulses from the electron multiplier. It has two redundant channels. Each channel has an 8-bit DAC controlled threshold. There is also a Faraday cup on the DET board whose current is read by a circuit on this board. 


\subsection{Thermal Design}

The SAM thermal design seeks to satisfy numerous heating and cooling requirements within a very resource-limited environment. Throughout development, the two largest constraints on the thermal design were power and volume. Because much of the gas transfer path (including numerous valve manifolds and sections of stainless steel or nickel tubing) must be heated to $135^{\circ} \mathrm{C}$ during SAM experiment sequences, considerable effort was expended on modeling and controlling heat losses. Contributing greatly to these losses is the Mars atmosphere itself. Although this atmosphere remains relatively stagnant within the SAM enclosure, heat leakage via convection and gas conduction precluded the use of multi-layer insulation blankets, which are normally effective in outer space. Insulation on SAM is largely achieved by utilizing low emittance surface finishes and attempting to maintain large $\mathrm{CO}_{2}$ "gas gaps" between components that operate at dissimilar temperatures. Finding adequate space for gas gaps within a densely packed instrument volume was often not possible, and this reality resulted in larger-than-anticipated power consumption for many heater circuits.

Mars atmosphere and surface temperatures vary widely with seasonal and diurnal cycles, and variations in wind, atmospheric turbidity, and levels of dust on the rover's external thermal radiators all produce great variability in the thermal environment. The primary heat sink for SAM is its mounting interface with the Rover Avionics Mounting Panel (RAMP). The RAMP serves as the primary mechanical/thermal interface for SAM and CheMin as well as most rover avionics boxes. Temperature of the RAMP is controlled via the rover's Heat Recovery System (HRS), a mechanically-pumped system which utilizes waste heat from the Radioisotope Thermoelectric Generator (RTG) to reduce the rover's need for heater power. The continuously circulating HRS fluid also helps to minimize thermal gradients and transients across the entire RAMP. SAM and the other components mounted to the RAMP are largely enclosed within the rover chassis and are shielded from Martian wind and dust. $\mathrm{CO}_{2}$ gas gaps between the outer walls of the rover chassis and SAM provide further insulation from the cold surface environment.

Several power-dissipating components within SAM require contact with a good thermal sink (i.e. the RAMP). These include the Main Electronics Box, RF Electronics, the two WRP's, the TLS TEC's, the HC-trap TEC's, and the GC injection trap TEC's. The MEB and HC-trap TEC baseplate are in direct contact with the RAMP, and an RTV thermal interface filler (NuSil CV-2942) is applied during SAM installation to ensure a high quality thermal contact. The RF Electronics is thermally connected to the RAMP via an encapsulated annealed pyrolytic graphite (APG) strap. Heat pipes transport heat from the four remaining components (TLS, WRP1, WRP2, GC TEC's). On the surface of Mars, these ammonia-charged heat pipes will operate in gravity-assisted (reflux) mode.

Since SAM is mounted within the interior of the rover, no survival heaters are required. However, a large number of SAM components do require active heating during experiment operations. These include the SSIT's $\left(\sim 120^{\circ} \mathrm{C}\right)$, the pyrolysis ovens (from ambient to 900 $1100{ }^{\circ} \mathrm{C}$ ), the WRP's $\left(>20{ }^{\circ} \mathrm{C}\right.$ for safe start-up), the TLS Herriott Cell (at least $-10{ }^{\circ} \mathrm{C}$ to maintain accurate composition measurements), the active gas path $\left(135^{\circ} \mathrm{C}\right.$ or greater to efficiently transport organic compounds from the oven to the GC and QMS instruments), the HC-trap (rapidly flashing to $>350{ }^{\circ} \mathrm{C}$ ), the GC injection traps (rapidly flashing to $\sim 350{ }^{\circ} \mathrm{C}$ ), the GC columns (ramping from ambient to $110-250{ }^{\circ} \mathrm{C}$ ), and several other items including the QMS ion source region, the $\mathrm{H}_{2} \mathrm{O}$ and $\mathrm{CO}_{2}$ scrubbers, and the getters.

The high temperatures required of many SAM components exceed the limits associated with "typical" spacecraft thermal control hardware, and much effort was invested in finding or developing materials and components that could be qualified for use on SAM. While 
conventional etched foil heaters are utilized for heating the TLS Herriott Cell and the SSIT funnels, the vast majority of heaters along the SAM gas transfer path are customized versions of the AeroRod BXX heater (ARI Industries). These Inconel-sheathed heaters are flexible and thin $(\mathrm{OD}=0.83 \mathrm{~mm})$ and can withstand temperatures in excess of $500{ }^{\circ} \mathrm{C}$. On SAM, they are typically wrapped in a spiral fashion around sections of tubing or pressed into grooves machined into the valve manifolds. In areas where sheathed heaters weren't suitable, simple resistance-wire heaters were manufactured in house. These heaters are utilized within the QMS source region, the GC capillary transfer lines, and the scrubbers. The heaters on a few SAM components are under PWM control, but most are electronically switched within the MEB. Although this would ordinarily provide simple on-off control, heater switching on SAM occurs at high speed (better than $1 \mathrm{~Hz}$ ) and PID control logic is implemented in flight software. Consequently, it was possible to "tune" the PID parameters for each heater circuit (PWM and switched) during suite-level environmental testing.

For temperature monitoring and control, SAM utilizes $60+$ flight temperature sensors including platinum RTDs (U.S. Sensor PPG501A1, rated to $500{ }^{\circ} \mathrm{C}$ ), glass-encapsulated thermistors (YSI $45006 \mathrm{H}$ type $10 \mathrm{k} \Omega$ and $44008 \mathrm{H}$ type $30 \mathrm{k} \Omega$ series, rated to $250{ }^{\circ} \mathrm{C}$ ), and board-mounted AD590 devices. The highest-temperature components with SAM (the pyrolysis ovens) utilize a long platinum alloy wire embedded within the oven heater block as a temperature sensor, and special tests were devised to calibrate this sensing element during development testing.

\subsection{Mechanical Structure}

The SAM structure is fabricated primarily from high strength T7075 aluminum alloy optimized for high strength to weight ratio. Gold plating was applied to the structure, which provides low emittance as a good thermal coating and good stability against flaking at high temperature. The structure was designed to optimize the limited volume allocated to the instrument suite, while maximizing the ability to integrate or remove any component during integration and test (I\&T) without disassembly of adjacent structures or components. Weight reduction was a primary driver of the design prompting the development of creative and unorthodox techniques to achieve the tight requirements. In some cases this involved utilizing existing adjacent hardware or structure to serve as the closeout or seal for the suite RF/EMI enclosure structures (Rover Payload Mounting Plate, SMS cover, etc.) rather than producing a more standard 6 sided box. Another means was to designate the primary structure support path such that the shear panels could be made into structural and non-structural areas for the overall closeout, yet provide the minimal required thickness of metal to shield the suite. Structural webs on the panels were typically $0.035^{\prime \prime}$ thick with non-structural areas as thin as $0.010^{\prime \prime}$; this required high-precision machining with the mill under vacuum to provide stability and maintain flatness.

\subsection{Flight Software}

The SAM flight software (FSW) is software embedded in the SAM instrument that controls all aspects of instrument operation. The FSW runs on a ColdFire microprocessor on the $\mathrm{CDH}$ board, and is in control of the instrument whenever the instrument is powered on. The FSW communicates with the MSL Rover over a bidirectional serial interface operating at $2 \mathrm{MBit} / \mathrm{s}$. Other serial interfaces allow the FSW to communicate with various SAM subsystems (GC, MOT, TLS). Control of the QMS and low-level hardware functions is through memory-mapped I/O on the $\mathrm{CDH}$ board. 
The FSW is in two components: the boot loader (approximately $30 \mathrm{kB}$ ) and the operational image (approximately $300 \mathrm{kB}$ ). The boot loader is burned in PROM, where it cannot be overwritten and is highly resistant to upsets. The operational image is stored in EEPROM, where it is written only under program control. This design allows the FSW to be safely modified or replaced, even during surface operations.

Boot Loader: The boot loader gains control when power is applied to the instrument. The boot loader nominally waits for $30 \mathrm{~s}$, then loads the operational image from EEPROM and transfers control to its entry point. However, if a command is received from the MSL Rover during the $30 \mathrm{~s}$ waiting period, the boot loader will abandon the timer and wait for commands. The boot loader has a full set of commands to maintain the EEPROM file system. It can create, modify, copy, move, or delete files; it can completely erase and reformat the EEPROM; it can consolidate files and mark bad blocks; and it can select among operational image files for one-time or default booting. The boot loader can also receive and load an operational image directly from the MSL Rover, without writing it first to EEPROM. The boot loader operates in a safe mode with interrupts disabled and the memory cache turned off. It does not contain an operating system; rather, it performs the necessary chip-level and board-level initializations and enters a background loop, listening for commands. The only exit from the background loop is via a program load operation.

EEPROM File System: SAM has the need to store a variety of parameter tables, script files, and other auxiliary information, in addition to its operational software image, in nonvolatile memory. The $1 \mathrm{MB}$ EEPROM, one of two nonvolatile memory stores in the CDH, is designated for this purpose. (The other is a $64 \mathrm{MB}$ flash memory array, used for telemetry storage as described below.) An EEPROM file system was developed specifically for use in the SAM FSW. The EEPROM memory is organized as a flat file system. Two directories are allocated at locations that are fixed when the EEPROM is formatted, nominally at the high and low end of the memory. The directories are redundant, with automatic fail-over. Directory entries are protected by checksums, and the file system will use the secondary directory entry for any given file if the primary entry is corrupted. In addition, all informationincluding both directories and files-is stored with an error-correcting code (ECC) that is capable of correcting any single-bit error and detecting any two-bit error in a 256-byte block. To guard against corruption by runaway code, the EEPROM is protected by a software write lock when not being written. The EEPROM file system is accessible by both the boot loader and the operational image.

Files occupy contiguous blocks of EEPROM memory. Each file is assigned a unique ID number when it is created. The ID number is used by the EEPROM maintenance commands (move, copy, delete, etc.) Each file has an assignable type code, which is used by the FSW to request a specific kind of file (e.g., a bootable image, a mass tuning parameter file, a MOT program load, etc.) without requiring knowledge of its ID number. A file is also given a file name, which appears in the directory listing and can be supplied as an argument to certain script commands, as well as aiding in human readability. A file checksum is computed when each file is written, and the checksum is stored in the file's directory entry. The file system will not permit the use of a file whose checksum on reading does not match the stored checksum. A directory listing is generated by the FSW at startup and on any commanded change to the file system. The directory listing appears in the message log telemetry. The directory listing includes ID, name, type, and checksum for each file, and aids in configuration management.

Flash File System and Telemetry: SAM is designed to operate autonomously for an entire experiment sequence of many hours duration. The data rate can vary during the course of the experiment sequence, but the total data volume is expected to be as much as $30 \mathrm{MB}$. 
During this time the MSL Rover may be powered off (to save energy), and operational constraints may dictate that SAM be powered off following the completion of the experiment sequence, with the expectation that it will be powered on to transmit its stored telemetry to the Rover at a later time. The $\mathrm{CDH}$ has a $64 \mathrm{MB}$ flash memory array for telemetry storage. Like EEPROM, data written to flash is retained across power cycles. However, flash memory supports many times more erase-write cycles than EEPROM and is better suited to autonomous operation.

A flash file system was developed specifically for SAM and incorporated into the FSW to handle the storage and retrieval of telemetry data in flash memory. SAM telemetry data is a continuous sequence of packets of varying origin within the instrument, but with a common packet structure. Each packet is time-tagged, and contains sufficient information to be processed by ground software independently of any other packet. Taking advantage of these characteristics, the flash file system organizes the memory as a FIFO. The file system maintains a read and a write pointer, and stores sufficient information in the flash metadata to reconstruct the two pointers even if power is abruptly disconnected. The locations of packet boundaries are also stored as the packets are written, so each read operation will always return an integral number of packets.

Like the EEPROM file system, the flash memory stores data with an error correcting code, which is capable of seamlessly correcting any single-bit error in a 256-byte block. The flash file system is capable of detecting and marking bad blocks, and the bad block status is preserved across power cycles. A flash memory scan at power-up rapidly detects bad blocks and locates the read and write pointers, so data storage is uninterrupted. Flash memory blocks are not erased until they are needed, so it is possible to recover lost data by "rewinding" the FIFO read pointer. Ordinarily, however, the read and write pointers continually cycle through the 64-MB flash memory, contributing to even wear over all flash memory blocks.

Telemetry data is read out of the flash file system under control of the Rover. Telemetry readout is performed when the rover computer transmits a "send telemetry" command to SAM, which is then iterated as long as there is telemetry data remaining in flash memory. In response to each command, SAM extracts packets from the flash read pointer until its telemetry buffer is full (approximately $32 \mathrm{~KB}$ ), and transmits the packets to the Rover. When the read pointer catches up to the write pointer, SAM returns a "no more data" indication and the iteration stops. During ground testing, SAM can be polled at regular intervals (e.g., a few seconds) to cause telemetry data to be delivered in real-time. Even during polling, however, the telemetry data passes through the flash memory as it does during surface operations.

Alarm Handler: The SAM FSW contains an alarm handler that is integrated with the script processor (see below). The alarm handler has continuous access to the more than 150 analog housekeeping values, hundreds of digital status points, and certain high-priority events such as the receipt of an "abort" command from the Rover. Integration with the script processor permits customized responses to individual alarms through scripted actions, without requiring specialized handlers within the flight software itself.

The alarm handler is configured by a table in EEPROM. The table specifies limits for analog and digital data points, and associates each limit with a scripted action. The action can be a script within the currently executing experiment sequence, in which case the experiment sequence continues after the alarm is handled, or it can be an external script, loaded from EEPROM, that stops the current activity and replaces it with the alarm handling activity. Since the original script cannot be resumed, these alarm handlers must make the instrument safe and ready to be powered off. Alarms are prioritized, to ensure that each event is fully processed, and to avoid recursion from multiple alarms. 
Script Processor: SAM's command system is a radical departure from prior spaceflight instrumentation. SAM is a BASIC interpreter. Its native command language encompasses the complete set of BASIC language constructs-FOR-NEXT, DO-WHILE, IF-ENDIF, GOTO and GOSUB - as well as a large set of unique built-in commands to perform all the specific functions necessary to configure and operate the instrument in all its possible modes.

SAM's commands, which are BASIC commands with SAM-specific commands built in, are transmitted in readable ASCII text. This eliminates the need for a binary translation layer within the instrument command flow, and makes it possible for operators to directly verify the commands being transmitted. There are more than 200 commands built in to the SAM BASIC script language. A lexical convention was established to group commands by subsystem (e.g., QMS, GC, TLS, SMS) to help organize the commands.

The majority of instrument commands offer low-level control over individual hardware components. The command set is designed to present a simple and complete hardware abstraction layer. In practice, however, few commands are used directly. Instead, sequences of low-level commands are grouped together to perform common tasks on the instrument. This is where the scripting capability is most useful. In BASIC, a "function" or "subroutine" can be defined, containing multiple lines of BASIC script, which may take arguments or parameters, and may return a value. These functions can then be called by name as if they were built-in commands. In this way, they become the building blocks used in the creation of the complex experiment sequences.

In SAM, a large collection of functions, subroutines, and variables, coded in BASIC and tested on instrument simulators, have been assembled in a BASIC script library file called "samlib.bas." This script library file is resident in SAM's EEPROM file system and available to all scripts. Samlib.bas defines 160 functions and contains over 3000 lines of BASIC script. A stripped-down version, with comments and excess white space removed, has been created for use in the flight system, occupying 87 kilobytes of nonvolatile storage on board.

Any SAM script can access the current values of all housekeeping and status data items. Telemetry data points are assigned numbers from 1 to 1000 for ground data system display and analysis. SAM BASIC has a predefined integer array, sam.hk(1000), in which each element is automatically populated with the current value of the corresponding data point. By reference to these values, a script can make decisions and control the experiment sequence flow based on the currently observed conditions in the instrument. This is an extremely powerful capability, which gives SAM scripts a level of control that far exceeds what could be accomplished through spacecraft command sequences.

The SAM BASIC commanding system introduces a new paradigm for complex instrumentation. It enables efficiencies in flight software development, instrument development, and operations. Flight software efficiencies arise from the capability of the script processor to combine and sequence hardware operations. With a highly capable script processor, flight software needs to provide only the hardware abstraction layer, i.e., a set of script commands that exercises every function and setting that the hardware is capable of taking on. Script commands are implemented in individual functions written in $\mathrm{C}$, using a standard calling convention. The correspondence between script commands and $\mathrm{C}$ functions is maintained in a table compiled in the software. BASIC internals take care of argument type checking and conversion between BASIC and C variable types. The actual implementation of hardware control functions is relatively straightforward, and the flight software can be developed and tested early in the instrument life cycle. In fact, this was the case with SAM, in which flight software led the flight hardware development even though the BASIC implementation itself was completely new for this instrument. 
The script processor also enabled efficiencies in the development of the instrument and its subsystems. Flight software supported component and subsystem testing without the need for special commands or modes. Scope loops and one-off tests were easily scripted, and a hardware regression test suite could also be developed and run as needed. Examples of the latter are the quick-look and electrical baseline tests that are run throughout the instrument life cycle to trend performance.

Finally, once the instrument was integrated and tested, the experiment sequences to be used in surface operations could be constructed from building blocks that were developed during component and subsystem testing. Thus, the language of operations and the language of flight software are different, and each is tailored to its purpose.

\subsection{SMS Calibration Cups}

The SAM SMS contains six individual metal calibration cups grouped together on the inner and outer rings (Fig. 5). One of the metal calibration cups was filled with $103.7 \mathrm{mg}$ of calcium carbonate powder (Sigma-Aldrich, ACS reagent, >99.95\% purity, \#398101) and capped with a porous $0.2 \mu \mathrm{m}$ metal frit. This calcium carbonate cup will be used by SAM to calibrate the evolved gas temperature for $\mathrm{CO}_{2}$ as measured by the QMS and the evolved $\mathrm{CO}_{2}$ will also serve as a calibration standard for carbon isotope measurements of $\mathrm{CO}_{2}$ measured by the TLS. The calcium carbonate standard was analyzed by a Costech ECS 4010 combustion elemental analyzer (EA) connected to a MAT 253 isotope ratio mass spectrometer (IRMS) and the $\mathrm{CO}_{2}$ released from the calcium carbonate had an average $\delta^{13} \mathrm{C}$ value of $-17.2 \pm 0.2 \%(N=3)$. The other five foil capped metal calibration cups contain a mixture of both calcium carbonate and fused silica (FS120, HP Technical Ceramics, LTD) and are sealed under vacuum via a pinch-off tube. The FS120 fused silica used in the calibration cups is the same material that was used for the Organic Check Material (OCM) canisters on the MSL rover. The FS120 used in the SAM calibration cups was powdered using a mortar and pestle, sieved to a 20 to $150 \mu \mathrm{m}$ particle size range, and then baked in air at $550{ }^{\circ} \mathrm{C}$ for 2 hours to remove any residual organic contamination prior to adding to the metal cup. One of the five foil capped metal cups contains only calcium carbonate $(3.7 \mathrm{mg})$ and FS120 powder $(121.7 \mathrm{mg})$. The other four metal calibration cups contain the same identical mixtures of calcium carbonate and FS120, but were spiked with $5.1 \mathrm{nmol}$ of 3-fluorophenanthrene (3FP, Chiron AS, 99.9\% neat, product \#1316.14) and $5.1 \mathrm{nmol}$ of 1-fluoronaphthalene (1FN, Sigma-Aldrich, $99 \%$ purity, product \#196657) prior to sealing by pinch-off under vacuum. The amounts of $1 \mathrm{FN}$ and $3 \mathrm{FP}$ in the calibration samples correspond to concentrations of 6 and 8 parts-per-million by total sample mass, respectively. The same fluorohydrocarbons were used to dope the Organic Check Material canisters used on MSL. These fluorohydrocarbon spiked solid sample calibrants will be run in situ on Mars in order to test the end-to-end pyrolysis GCMS mode of SAM. The SAM pyrolysis evolved gas and pyrolysis GCMS experimental modes were tested extensively in a Mars chamber using mixtures of calcium carbonate $\left(\mathrm{CaCO}_{3}, 3.7 \mathrm{mg}\right)$, melanterite $\left(\mathrm{FeSO}_{4} \cdot 7 \mathrm{H}_{2} \mathrm{O}, 3.5 \mathrm{mg}\right)$, and FS120 (42.8 mg) that were spiked with similar concentrations of 1FN and 3FP.

\subsection{Gas Calibration Cell}

The SAM calibration gas cell $(4.76 \mathrm{ml})$ is used to monitor for shifts in performance over the long duration of this mission. It will also be used for tuning the QMS and providing a characterized reference gas for isotopic analyses of martian gas by both the QMS and TLS.

The calibration gas cell contains a mix of four primary gases plus trace quantities of four higher-molecular-weight compounds (Table 11). The calibration gas mix is composed of 
Table 11 Components of the SAM calibration gas cell

\begin{tabular}{lllcc}
\hline Abbrev & Chemical & Purity & Mol.wt. & $100{ }^{\circ} \mathrm{C} \mathrm{MR}$ \\
\hline $\mathrm{CO}_{2}$ & Carbon dioxide & $99.99 \%$ & 44 & $24.32 \%$ \\
$\mathrm{~N}_{2}$ & Nitrogen & $99.9995 \%$ & 28 & $24.10 \%$ \\
$\mathrm{Ar}$ & Argon & $99.999 \%$ & 40 & $24.04 \%$ \\
$\mathrm{Xe}_{\mathrm{T}}$ & Xenon (near terrestrial isotopic composition) & Research grade & 131 & $8.48 \%$ \\
${ }^{129} \mathrm{Xe}$ & 129-Xenon & $(88.9$ atom $\%)$ & 129 & $15.51 \%$ \\
PFTBA & Perfluorotributylamine & Not available & 671 & $3.00 \%$ \\
1 FN & 1-Fluoronaphthalene & $99 \%$ & 146.161 & $0.54 \%$ \\
DFBP & 2,2'-Difluoro-1,1'-biphenyl & $98 \%$ & 190.189 & $0.016 \%$ \\
PFBP & Perfluorobiphenyl & $\geq 98.0 \%$ & 334.112 & $0.0078 \%$ \\
\hline
\end{tabular}

Fig. 17 Structures of calibration compounds

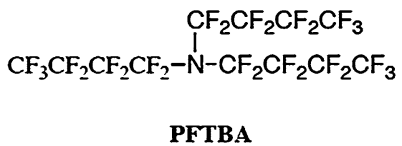<smiles>Fc1cccc2ccccc12</smiles>

1-FN

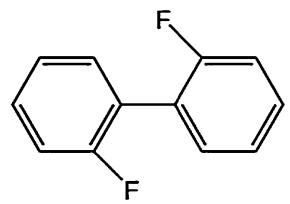

DFBP<smiles>Fc1c(F)c(F)c(-c2c(F)c(F)c(F)c(F)c2F)c(F)c1F</smiles>

PFBP

$\sim 25 \%$ each of high purity $\mathrm{CO}_{2}, \mathrm{~N}_{2}$, Ar, and Xe. A three-fold enrichment of ${ }^{129} \mathrm{Xe}$ relative to terrestrial $\mathrm{Xe}$ was used in order to produce a Xe isotopic composition strongly different from the low ${ }^{129} \mathrm{Xe} /{ }^{132} \mathrm{Xe}$ composition modeled for Mars' atmosphere $\left({ }^{129} \mathrm{Xe} /{ }^{132} \mathrm{Xe}<1.07\right.$; Mathew and Marti 2001).

The four higher-molecular-weight compounds are fully to partially fluorinated compounds, which are not formed naturally, thus making them readily distinguishable as terrestrial organics during MSL operations. The compounds chosen for the calibration gas include perfluorotributylamine (PFTBA), a standard for mass calibration and sensitivity checks during QMS tuning, 1-fluoronaphthalene (1FN), 2,2'-difluoro-1,1'-biphenyl (DFBP), and perfluorobiphenyl (PFBP) (Fig. 17). The latter three compounds serve as additional standards for checking peak resolution and relative retention times on the four GC columns designed for medium to high molecular weight organics (MTX-20, MTX-5, ChiralDex, and MTXCLP). In addition, 1FN in the calibration cell provides an independent detection test for the more volatile standard in the OCM. Differences in the molar ratio may be used as an independent, compound-specific sensitivity check via the GCs. The 1FN, DFBP, and PFBP are 
at least one order of magnitude less abundant than PFTBA and do not interfere with mass tuning of the RF of the QMS.

During transfer of material from the calibration cell, the cell temperature will be raised to $100{ }^{\circ} \mathrm{C}$ or higher to insure that all components are fully in the gas phase so that a known gas mix ratio can be introduced into the manifold. A flow restrictor associated with V36 enables a known volume of gas to be introduced into the manifold by for a selected time that this valve is set to be open. The installation pressure for the calibration gas cell was 1.3 bar at the targeted gas cell temperature of $125^{\circ} \mathrm{C}$. The relative abundances of the fluorocarbons in the calibration cell were selected to provide a variation of more than two orders of magnitude. This enables the limits of detection of the GCMS to well below $0.1 \mathrm{nmol}$ to be readily confirmed using on-board calibrants. The calibration gas cell will support at least $100 \mathrm{such}$ in situ calibration experiments.

\subsection{Organic Check Material (OCM)}

The OCM consists of 5 hermetically sealed metal vessels containing quantified and trace levels of distinctive fluorocarbon compounds distributed in a fused silica glass matrix. The OCM will be used on Mars for an end-to-end check of the state of the sample transfer chain with regard to terrestrial contamination, including the SAM SSITs. The detailed requirements, design, and expected usage of the OCM are described by another manuscript (Conrad et al.) in this issue.

\section{SAM Development Approach}

\subsection{Overview of Systems Engineering Processes}

A set of systems engineering processes are mandated by NASA/GSFC in the development and implementation of spaceflight hardware. Although the list of NASA Procedural Requirements (NPRs) is extensive, most of the important Systems guidelines are documented in:

1. NPR 7120.5, the NASA Space Flight Program and Project Management Requirements

2. NPR 7123.1; NASA Systems Engineering Processes and Requirements

3. GSFC-STD-7000; General Environmental Verification Standard (GEVS)

4. GSFC-STD-1000; Rules for the Design, Development, Verification, and Operation of Flight Systems (GOLD Rules)

Adherence to the mandated requirements is evaluated at the Project Reviews that conclude each Project Lifecycle phase (Fig. 18).

\subsection{Integration and Test Considerations}

The Electrical Baseline Test (EBT): A battery of SAM tests were performed during the various phases of pre-launch development. The one run most often was the EBT; a fully automated serial check of over 1000 electrical circuits (test the wire) that ran in $\sim 33$ minutes.

The Comprehensive Performance Test $(C P T)$ : This test mimics the two most common SAM experiments to be run on Mars: the atmospheric sample analysis, and the core solid sample analysis sequence. The tests are run from scripts that are identical to those that will 


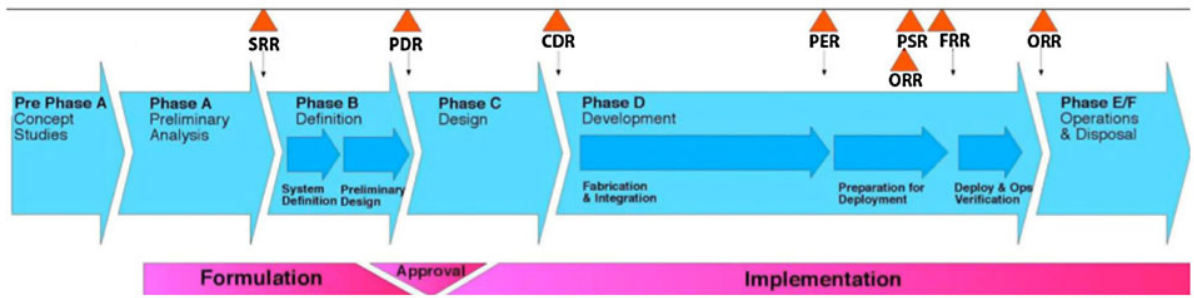

Fig. 18 Project phases and reviews detailed in the Systems Engineering Handbook (SP-6105) and the NASA Project Lifecycle (NPG-7120). Major project independent reviews for SAM are designated as follows: SRR (Systems Requirements Review), PDR (Preliminary Design Review), CDR (Critical Design Review), PER (Pre-Environmental Review), ORR (Operational Readiness Review), and FRR (Flight Readiness Review)

be run on Mars. Several one-off tests that verified related processes were built from the CPT, including calibration tests. The repeated runs of the CPT over time and temperature ranges allowed for functional unit performance, as well as scientific system performance to be analyzed and trended for patterns of change.

Test as you Fly: With very few exceptions tests were designed to be duplicates of the runs to be made on Mars. The drop of a solid sample under Mars gravity was an exception. Extensive analysis of flow, seals, joints, and transitions was used for sample drop verification. Key to the test-as-you-fly philosophy was the design of the Mars Chamber. This chamber was designed to duplicate the thermal interface to the MSL Rover both conductively and radiatively. The conductive thermal plate was built and delivered by the Rover team to Rover flight drawings. The chamber pressure is adjustable to the range of Mars pressures as specified by MSL for the range of mission locations since during SAM tests, the exact landing site was not yet selected.

Testbed Development: A duplicate of the Flight Model SAM Instrument suite is in fabrication as a Testbed. The SAM Testbed will be utilized to exercise experiment sequences that were not exercised on the SAM FM in order to conserve consumables on the flight unit or in order not to introduce an excess of terrestrial material into the SAM FM Differences between the Testbed and the Flight Model are well documented minimized. For instance the solid sample quartz cups are expensive, so the Testbed is populated with only 20 cups while the FM contains 59 quartz cups. The Testbed will stay resident in the Mars Chamber during the Mission, running a parallel mission on Earth. The Mars Chamber can provide a highly robust and controllable simulation of the environment encountered by the SAM FM on Mars. When a script is substantially changed to run a variation of an experiment sequence that has not run before, it will be run on the Testbed, before it is run on the Flight instrument to maximize the chance for a successful completion of the experiment on Mars. In addition, if flight spectral mass data points to ambiguous or uncertain conclusions, the Testbed can be loaded with an analogous analog sample and processed as on Mars to correlate with the flight data. Data archives developed during test are resident on servers for distribution to scientists and engineers to process according to their interest. The SAM Testbed telemetry and data structure is identical to that of the SAM FM and the analysis of Testbed data can utilize the extensive set of software tools developed for the processing of the SAM FM data. 


\section{SAM Qualification and Pre-Launch Calibration}

\subsection{Verification and Calibration Approach}

The complexity of the SAM suite motivated a rigorous test and verification program at several stages of development prior to integration and test at the suite level and subsequently in the rover after delivery to the Jet Propulsion Laboratory. Qualification units of several components such as high conductance valves, micro-valves, heaters, ovens, traps, and getters were tested under relevant environments to their predicted lifetime times a safety factor of 1.5 or greater in the nominal landed mission. Although thermal stress analysis was carried out on all electronic boards, each board was also subjected to an extensive set of operational tests at temperatures that spanned the required environmental test conditions required for qualification at the suite level. Vibration tests were carried out on many components such as the QMS, the TLS, the GC, the MEB, and the HC-trap prior to their integration into the suite. The suite level tests at the NASA GSFC prior to delivery of SAM included vibration of the suite at proto-flight qualification levels and sine burst tests on the lateral axes ( $X$ and $Y$ ) to $12.5 \mathrm{~g}$ 's and in the thrust $(Z)$ axis to 18.75 to test for the most stringent Mars entry load conditions. Vibration tests of the suite and most components and shock tests of the suite were carried out in the NASA GSFC multi-project facilities (Fig. 19) while EMI and thermal tests were carried out the NASA GSFC building 33 facilities of the Planetary Environments Laboratory. During vibration 24 accelerometers were attached to components and critical locations on the suite to monitor levels and during thermal tests many thermocouples were similarly integrated to closely monitor the temperature response through the suite. These thermal and vibration tests at the suite level uncovered a small number of issues such as a mechanical failure in the bellows attaching a getter to the mass spectrometer, failure of a vacuum feedthrough to one of the SAM ovens, and a workmanship error in the SAM high voltage module. These issues at the suite level while straightforward to correct were time consuming because of the suite disassembly needed to remove and repair the components and the subsequent retesting of the suite. Although redesigns of the WRP rotor/shaft mating configuration and its bearing retainer were in hand well before delivery of the SAM suite to JPL, the required lifetime testing of this pump stretched out until it successfully reached $2 \times$ the nominal surface lifetime just prior to SAM delivery. SAM was delivered to JPL in December 2010 without impact on the assembly schedule for the rover.

Fig. 19 SAM instrument is shown being lowered on to the vibration fixture

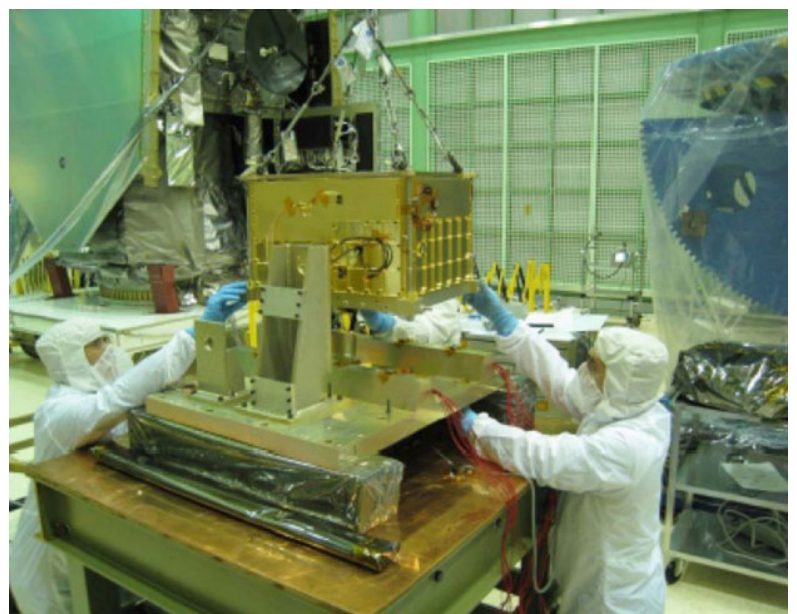



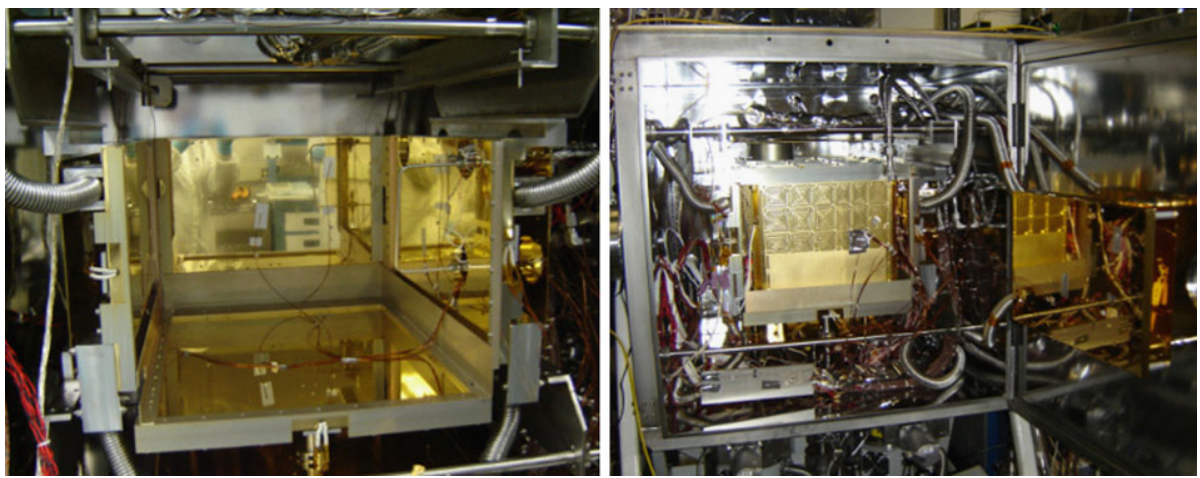

Fig. 20 Picture of the SAM environmental/calibration chamber with (right) and without (left) SAM in place. The chamber itself is a cube approximately 1 meter a side

The SAM environmental chamber (Fig. 20) was developed to carry out both thermal testing and qualification and calibration in an environment that could simulate the thermal conditions in the rover on the surface of Mars with high fidelity. For efficiency, the sequence of thermal cycling required for suite qualification was combined with a required set of calibration experiments utilizing both solid samples in the SMS and atmospheric samples introduced into SAM through chamber feedthroughs from a gas processing system external to the chamber. The final set of calibration/thermal vacuum tests prior to delivery of the suite to JPL consisted of a series of EGA/GCMS/TLS experiments with SAM at the ambient temperature of $20{ }^{\circ} \mathrm{C}$ followed by a series of 6 hot/cold excursions in a Mars $\mathrm{CO}_{2}$ pressure environment. On the high temperature end of each hot cycle $\left(50{ }^{\circ} \mathrm{C}\right)$ and the low temperature end of each cold cycle $\left(-40^{\circ} \mathrm{C}\right)$ a standard Electrical Baseline Test (EBT) was run that tested all of the SAM electrical functions that were possible to test without turning on the pumps or the instruments. At temperatures of $+45^{\circ} \mathrm{C},+25^{\circ} \mathrm{C}$, and $-20{ }^{\circ} \mathrm{C}$ core atmospheric and solid sample sequences identical to those that could be run on Mars were exercised. These tests were most valuable in establishing energy requirements for SAM as a function of temperature and practical suite temperature operational limits for some of the temperature sensitive items such as the TLS methane laser or the GC TCD detectors.

\subsection{SAM Mars Environment Chamber}

Chamber Overview: The SAM instrument Mars chamber is located in the planetary environments laboratory of at NASA GSFC. The SAM chamber is a 36" electro-polished stainless steel cube fitted with an internal thermal shroud. The system is capable of vacuum below $10^{-7}$ Torr after extended bake-out as well as dynamic atmospheric pressure control. The thermal shroud internal test volume of $26^{\prime \prime} \times 22^{\prime \prime} \times 16^{\prime \prime}$ is comprised of 6 independent thermal zones. The Mars chamber is equipped with a dedicated 120 channel thermocouple data acquisition system and standard contamination control and monitoring systems to include a Thermoelectric Quartz Crystal Microbalance (TQCM), Residual Gas Analyzer (RGA) and scavenger plate.

Vacuum System: Special attention was given to all interior surfaces and materials in construction of the Mars chamber. All metal Conflat seals, electro and gold plated walls, structure, shrouds and oil free vacuum pumps were utilized. The chamber rough vacuum system 
is comprised of a Pfeiffer 150-2 Extra-Dry pump and high conductance right angle valve. The roughing system is used to evacuate the chamber to less than $1 \times 10^{-1}$ Torr prior to high vacuum cross-over. High vacuum operations are supported by two Pheiffer 521 turbo molecular pumps which are backed by independent 150-2 Extra-Dry mechanical pumps. The bottom wall of the chamber is equipped with two VAT 8 " Conflat gate valves for turbo pump isolation. The vacuum system is fitted with a dynamic Mars atmospheric control feature. The system is comprised of an external Mars mix gas cylinder and 2 stage pressure regulator piped to the chamber volume through a $1 \times 10^{-2}$ atm cc/sec flow restrictor. Chamber pressure balance is maintained by a variable conductance metering valve attached to an Adixen Drytel 1025 molecular drag pump.

Thermal System: Chamber thermal control is accomplished by $51^{\prime \prime}$ thick gold plated copper thermal plates with serpentine gaseous nitrogen plumbing loops and embedded cartridge heaters as well as 1 high fidelity thermal plate controlled by an external re-circulating chiller. The high fidelity plate was designed to mimic in great detail the SAM thermal interface of the Rover Avionics Mounting Plate (RAMP). The RAMP plate is capable of 300 Watts of heat rejection from $-55^{\circ} \mathrm{C}$ to $+70{ }^{\circ} \mathrm{C}$. A SAM instrument thermal/mass mockup was used to calibrate the RAMP simulator prior to SAM testing. Five independently controlled gold plated copper thermal plates are used to simulate the rover cavity walls surrounding the SAM instrument. An external 240 Liter LN2 dewar was utilized as a phase separator to serve as a high capacity cold gas source. An ambient temperature GN2 line was routed from the main building LN2 tank and regulated down to 20 PSI as a heat input to the LN2 dewar. This warm gas source is reverse fed through the liquid in/out valve and boils through the internal liquid volume. Cold gas is extracted from the dewar vent valve and transferred via vacuum jacketed transfer line to the chamber gas distribution manifold. Cooling gas is distributed by PID-controlled cryogenic solenoid valves to the individual plates. All chamber surfaces must remain above $-125{ }^{\circ} \mathrm{C}$ when operating with a Mars atmosphere to prevent condensation of the $\mathrm{CO}_{2}$.

Gas Distribution: The SAM instrument has multiple atmospheric inlets, vents and a solid sample processing system. A major component of SAM testing and calibration involves the introduction of calibration gases and simulation of Mars atmospheric pressure at the vents of the wide range turbo pumps. This feature is supported by a complex external pumping system comprised of 2 Pheiffer 261 turbo pump carts, 2 Drytel 1025 molecular drag pumps and 25 valve manifold.

Data Acquisition: The Thermal Vacuum Data System (TVDS) is a 120 channel standalone system providing 24/7 monitoring and alarming of facility and flight hardware temperatures. Temperature measurements are made by a Keithley 2750 data acquisition system commanded by an Agilent VEE software application. All test data is archived to an Oracle database running on a RAID 5 windows server. Real time and historical data are available in tabular and graphical formats. An addition script running on the SAM instrument EGSE supports the logging of flight telemetry to the Oracle TVDS.

\subsection{Electrical Ground Support Equipment}

Several MSL rover simulators were developed for SAM testing and for operation of SAM in calibration and in qualification testing either at the NASA GSFC, JPL, or Cape Canaveral. The rover simulator generated power on each of the SAM buses over the range of voltages expected from the rover. The simulator included GSE to enable accurate measurements of SAM power consumed to be made over the course of an experiment. The simulator also included a high fidelity data interface and a clock that allowed a measure of the ON time for the SAM suite. 


\subsection{Contamination Control}

A substantial driver for both SAM fabrication methods and measurement protocols was the requirement to measure parts per billion by mass of organic compounds in martian samples without confusion from terrestrial contamination. Most sample path hardware was processed with a rigorous toluene, acetone, detergent, deionized water, alcohol ultrasonic cleaning process with many metal components receiving a $200{ }^{\circ} \mathrm{C}$ or higher vacuum bake. Special handling protocols were implemented for the most critical components with UHV aluminum foil utilized as the first surface contact for packaging and bagging for transport to the SAM integration area. Assembly of the QMS was carried out separately in a Laminar flow bench and after assembly the QMS was separately baked at temperatures greater than $200{ }^{\circ} \mathrm{C}$ for several days. Initial assembly of the SAM suite was in a class 1000 cleanroom where the assembly bench where most of the work on SAM was carried out was typically class 100 . Full gowning protocols were required of the assembly team. Major subassemblies required outgassing certification to a level determined by the proximity of that element to the sample processing chain. Surface external to the sample processing chain were required to be cleaned to a non-volatile residue level of $<500 \mathrm{ng} / \mathrm{cm}^{2}$ and a particle level of VC-II (inspected under black light conditions) or L300 (<93 particles in the 100-250 micrometers, $<3$ particles in the 250-300 micrometer, and no particles $>300$ micrometers per square 0.1 meter).

Once the suite was mostly assembled prior to final installation of the SAM WRP's, the gas manifolds and the QMS were rigorously baked for many days (temperatures mostly between $150-200{ }^{\circ} \mathrm{C}$ ). During this period the WRP's were replaced with commercial laboratory ultra-clean turbomolecular pumps. The gas manifold was periodically purged with $\mathrm{CO}_{2}$ and organic compounds flushed out of the system were periodically trapped on a Tenax trap and analyzed on sensitive laboratory GCMS instruments. After the final suite assembly, it was baked in the calibration chamber for $\sim 75$ hours and the outgassing monitored with quartz microbalances (QCM's). The QCM trend line established at $50{ }^{\circ} \mathrm{C}$ generated a predicted TQCM rate of $\sim 35 \mathrm{~Hz} / \mathrm{hr}$. Since there was no direct path from the SAM electronics and other external components to the SAM sample transport path, this level was judged sufficiently low so as to be in the noise of the contribution from the avionics, the structure, and other payload elements of the rover.

The best verification of SAM internal cleanliness was the SAM instrument itself. The measurement protocol on Mars requires a quartz cup to be pre-cleaned to $>900{ }^{\circ} \mathrm{C}$ and the cup left sealed in the ultra-clean environment of the oven until a sample is ready for delivery. Only then is the cup moved over to the SSIT to accept sample and returned to the oven within a few minutes and then sealed. Tests were carried out during qualification and calibration of this protocol. When this protocol was followed the nano-moles or sub-nanomoles of calibrant materials dominated the GCMS spectra. On the other hand, when a SAM quartz cup was utilized without this pre-cleaning step significant levels of contamination were observed in the spectra. These experiments are useful as they enable the nature of the terrestrial organic component in the SMS that eventually can diffuse to adsorb on a SAM cup to be characterized.

Shortly before launch the SA/SPaH team at JPL uncovered in laboratory engineering test units a source of contamination originating from the rotary/percussive drill element of this system. A partition diaphragm in the interior of the drill system releases particles of Teflon and molybdenum disulfide lubricant into the sample chain. A portion of these particles makes their way through the $150 \mu \mathrm{m}$ sieve and would contaminate samples delivered to the SAM cups if the identical behavior were to occur on the flight SA/SPaH. Studies to 
understand the potential impact of this contamination on the SAM GCMS analysis and to explore mitigation strategies are presently ongoing.

\subsection{The SAM Experiment Simulator}

The SAM Experiment Simulator (SAMSIM) is a numerical model dedicated to help plan operations during the commissioning and science phases of the mission by providing predicts of the instrument electrical, thermal, mechanical and fluid responses to scripted commands. The SAMSIM model was developed using the Matlab and Simulink libraries of Mathworks. Matlab and Simulink form a widely used platform for multi-domain simulation and modelbased design of dynamical systems. This platform offers also an interactive graphical environment and a customizable set of block libraries that let the user easily design, implement, and validate models of complex time varying systems.

The SAMSIM model is divided into four distinct but interacting modules.

The Software Module: This element simulates the instrument Command and Data Handling System $(\mathrm{C} \& \mathrm{DH})$ by executing in a Matlab environment a copy of the actual instrument flight software. The outputs of this synthetic C\&DH are mapped to virtual sensors and command busses that mimic in their structure and connectivity the layout of the instrument harnesses. This module can execute, and thus validate, command scripts that are identical to those that can be executed by the SAM instrument. This module will also generate synthetic data at a rate that is similar to the actual instrument.

The Mechanical Module: This element simulates the actions of a number of mechanical subsystems of the SAM instrument. This module contains the valves, the pumps, and the SMS. The response of each of these elements is modeled to produce outputs of state variables (velocity, position, force, electric currents, etc.) that duplicate the behavior of the real elements.

The Thermal Module simulates the action of each of the many heaters, and their thermal impact on the instrument as measured by SAM's $\sim 60$ thermistors and as it is seen by the $\mathrm{C} \& \mathrm{DH}$. The thermal behavior of each element in this module is modeled as a linear parametric function. The coefficients of these parametric functions were identified by using real thermal data gathered during the testing phase of the instrument in a controlled environment chamber.

The Flow Module simulates the gas dynamics (time dependant pressures and gas flows) in the gas processing system of SAM. This module was built using a Simulink electrical analogue to each element of the gas flow system. The conductance of each gas transfer element is dynamically computed using the Knudsen empirical formula (Delchar 1993) as a function of the element dimension, gas composition, pressure and temperature. Quantitative comparison between the model and the actual gas processing system of SAM showed that this formula provides gas flow parameters with better than $20 \%$ accuracy. This module was utilized numerous times during the development of the SAM instrument to size flow restrictors and calculate gas loads and pressures.

The Electrical Module captures the electrical behavior of motor control boards and all elements that contribute to the instrument power profile. Each element's electric performance is modeled using either a parametric formula or a lookup table.

The behavior of each element of the SAMSIM model is reconstructed by accounting for all the relevant modules for that element. Complex elements like pumps use contributions from all these modules to simulate the evolution of their command logic, rotor speeds, currents, temperatures, and throughputs. 
The SAMSIM model was extensively used during the instrument testing and calibration in the laboratory and will be deployed as one of the ground support tools during the commissioning and science operations on Mars. The model will help validate each new script that will be uploaded to the instrument and will provide predicts of the time duration and power profile that will be needed and the data volume that will be generated by the instrument. The model will also track the usage of consumables and will be used to follow trends and any performance degradation that may occur as the instrument ages.

\subsection{SAM Resource Utilization}

The most challenging MSL resource that will fold into mission operations planning is the SAM energy budget. The SAM solid sample ovens, the GC and manifold heaters, the turbomolecular pumps and the energy associated with the TEC's on the TLS and the RF on the QMS are all drivers in this regard. The energy consumed during any given sol for a SAM activity can vary greatly depending on the particular analysis script selected and the time of year, time of day, and other factors such as winds and atmospheric opacity that determine the thermal boundary conditions for SAM. For this reason the energy intensive sequence of cleaning a quartz sample cup, delivering sample to this cup, and implementing a full EGA/GCMS/TLS analysis is split into three sols. In the first sol the cup is preconditioned, in the second sol the sample is acquired and delivered, and in the third sol the SAM analysis takes place. Atmospheric analysis will typically utilize considerably less energy. Considerable optimization has been done, to date, to reduce the energy consumption of SAM. The suite incorporates a power distribution box for heaters which avoids the overhead of going through the main SAM power supply and letting multiple elements of the system more rapidly achieve their operating temperature. For example, the WRPs are not turned on until they are heated to $20^{\circ} \mathrm{C}$ to allow the elastomer o-rings in the system to reach the necessary viscoelastic properties for robust pump operation. Typically manifolds and transfer plumbing lines are heated from $135^{\circ} \mathrm{C}$ to $175^{\circ} \mathrm{C}$ to allow for more efficient transport of organic compounds from the oven to the GC columns. Figure 21 shows a power profile for the full EGA/GCMS/TLS sequence of operations in a cold $\left(-30^{\circ} \mathrm{C}\right)$ RAMP interface environment for each of the three MSL power busses that service SAM. This is a typical worst case utilization of power, data, and experiment duration resources with a total energy consumption of $\sim 1050$ watt-hours, a data volume of $\sim 11.8$ Mega bytes, and a duration of $\sim 6$ hours.

Fig. 21 A power profile for a full EGA/GCMS/TLS sequence on a $-30{ }^{\circ} \mathrm{C}$ SAM RAMP thermal interface temperature is shown. This data was measured during SAM thermal vacuum qualification

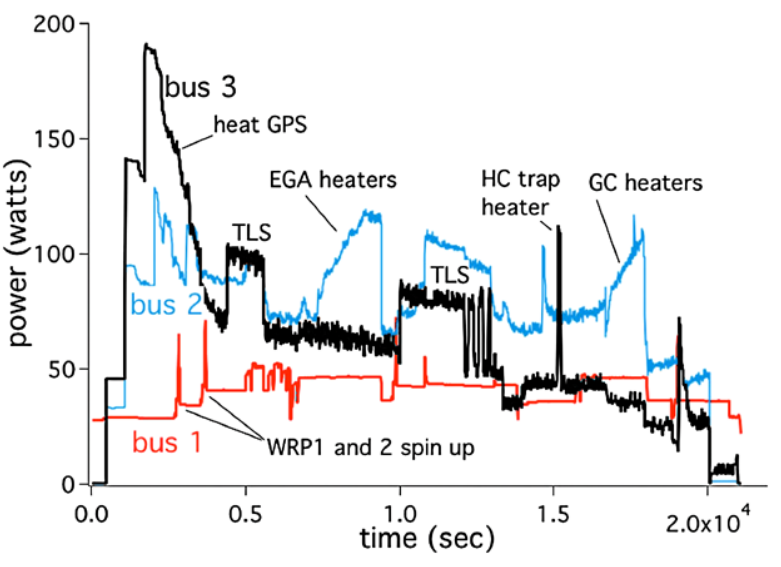




\subsection{SAM Breadboards Developed for Analog Studies}

Several laboratory instrument breadboards have been developed to represent various SAM subsystems and model the performance of the flight unit without subjecting it to unnecessary contamination or use of consumable resources such as the lifetime of the WRP. The highest fidelity QMS breadboard utilizes a mass spectrometer nearly identical to the SAM FM, controlled by a high fidelity RF electronics breadboard circuit driven by a breadboard of the FM CPU. The mass spectrometer can be pumped either by a commercial pump or by a flight like WRP. For an EGA experiment a sample in a quartz cup can be heated with the same temperature profile as in the SAM FM oven and with the same pressure and flow rate of the carrier helium gas (Franz et al. 2011). Another breadboard in the Planetary Environments Laboratory at the NASA GSFC utilizes a similar oven and similar temperature and carrier gas conditions but replaces the SAM QMS breadboard with a commercial instrument (McAdam et al. 2011). Analogous units utilized for studies related to the TEGA instrument of the Phoenix Scout Mission have been adapted to SAM EGA conditions by SAM team members at the NASA Johnson Space Flight Center. A laboratory analog for the TLS is a commercial cavity ringdown spectrometer (CRDS) that measures $\delta^{13} \mathrm{C}_{\text {in }} \mathrm{CO}_{2}$ in atmospheric gas or gas evolved from a solid sample on heating, with precisions approaching that of the TLS (Stern et al. 2011). Ground truth for these isotope measurements is provided by a commercial isotope ratio mass spectrometer (IRMS). Several commercial pyrolysis-GCMS breadboards include modules that utilize identical or similar columns as the flight GC combined with injection traps of the same design. These breadboards are being used for wet-chemical processing and inert pyrolysis experiments involving standards and analogs.

\subsection{Mineral and Analogues EGA Library}

A SAM library of EGA analyses from pure mineral reference materials and Mars analog samples is under development. Much of the data in this library is acquired under SAM EGA conditions of ramp rate and helium flow and the experiment conditions and breadboard instruments used are documented. For many samples, geochemical characterization by XRD/XRF or other analytical methods has also taken place and this data is included. Pure mineral samples included in the EGA library may be either natural mineral reference materials or synthetic analogs, such as the calcite and melanterite used for flight model validation. Analysis of pure single minerals makes the interpretation of the EGA data as straightforward as possible by reducing the number and extent of chemical reactions during the EGA process that can shift peak positions and create new reaction products. To assess these effects, the library will include data from experiments using specific combinations of minerals and natural analog materials for comparison to data from single mineral runs. Figure 22 shows example EGA signals from selected $\mathrm{m} / z$ values for several of these experiments.

The top frame of Fig. 22 compares the evolved gas curves obtained on the SAM breadboard for two carbonates. The shift in the peak temperature is illustrative of the type of information that can often be used to distinguish between mineral types. Calcite, not shown in this plot, releases $\mathrm{CO}_{2}$ at even higher temperatures. The quantity of $\mathrm{CO}_{2}$ evolved in these examples, which to first order is proportional to the area under the $\mathrm{CO}_{2}$ curve can be used to quantitatively determine the molar quantity of carbonate present. In addition, the SAM calibration experiments with the FM QMS have demonstrated that the sensitivity of the SAM QMS will enable quantities down to micrograms (or parts per million mixing ration by mass for a typical volume of sample delivered to a SAM cup) to be detected. The middle frame of 
Fig. 22 Evolution of $\mathrm{CO}_{2}, \mathrm{SO}_{2}$, and $\mathrm{H}_{2} \mathrm{O}$ with temperature from selected minerals in the SAM breadboard. Temperature ramps and helium carrier gas flow and pressure were controlled to nominal values for the SAM landed mission. A library of such data is being built for use in the interpretation of SAM data
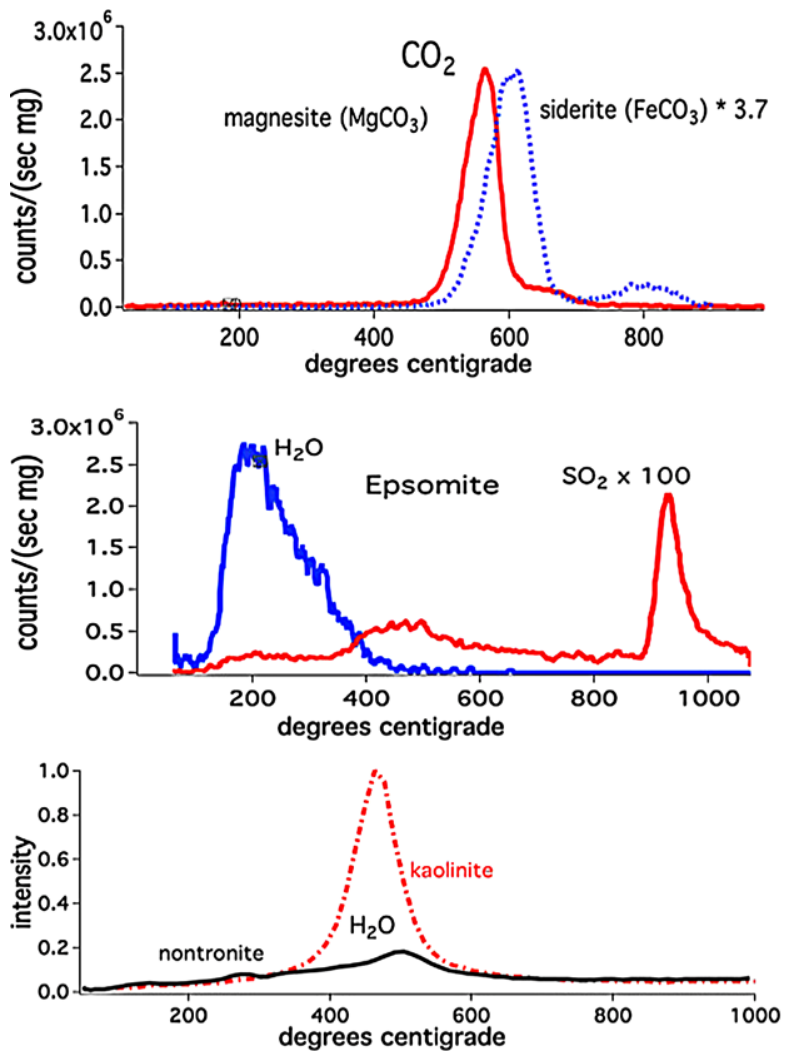

Fig. 22 shows both the $\mathrm{SO}_{2}$ and $\mathrm{H}_{2} \mathrm{O}$ evolution curves resulting from the thermal processing of melanterite also under SAM helium carrier gas flow and pressure and temperature ramp rate conditions. Water of hydration is released early, peaking at $\sim 200{ }^{\circ} \mathrm{C}$ and the $\mathrm{SO}_{2}$ released from the decomposition of the sulfate is released above $900{ }^{\circ} \mathrm{C}$. The bottom frame of Fig. 22 illustrates the high temperature release of water from two clays. The evolved gas curves secured with the SAM QMS will enable the temperature range to be bracketed for introduction of gas into the TLS during an evolved gas experiment. The TLS data will further quantify the amount of water or carbon dioxide released from the solid sample and will the full isotopic composition of these molecules to be determined. Background water in the QMS will be the limiting factor in its sensitivity for water release from hydrated minerals. The SAM sensitivity for $\mathrm{SO}_{2}$ release from many sulfur containing minerals is hundreds of nano-grams even better than that established for $\mathrm{CO}_{2}$ since the SAM pumps in this case will not have an instrument background due to backstreaming through the WRP as in the case of $\mathrm{CO}_{2}$ for the carbonate measurements. Table 12 lists some of the reference materials analyzed by these EGA runs.

\subsection{Calibration Analysis Methods and Representative Data}

Each instrument was tested and calibrated prior to delivery to the SAM integration team and then the full suite was then calibrated in the Mars-like chamber environment with a variety of samples over a range of environmental conditions. 
Table 12 Mineral reference materials analyzed by SAM-like EGA

\begin{tabular}{ll}
\hline Mineral type & Specific minerals \\
\hline Carbonates & Calcite \\
& Magnesite \\
Sulfates & Siderite \\
& Melanterite \\
& Gypsum \\
& Jarosite \\
Oxides and oxyhydroxides & Kieserite \\
Sulfide & Epsomite \\
Phyllosilicates & Hematite \\
& Goethite \\
Poorly ordered aluminosilicate & Pyrite \\
Perchlorates & Nontronite \\
& Montmorillonite \\
& Chamosite \\
& Kaolinite \\
& Illite \\
& Allophane \\
& Mg-perchlorate \\
\hline
\end{tabular}

TLS Calibration: Calibration of TLS has been done in a variety of stages, both as a standalone instrument during pre-delivery thermal-vacuum testing, and more thoroughly during suite-level calibrations using NIST-traceable standard calibration gases over a wide variety of operating temperatures and pressures. In addition, TLS carries a reference gas cell for the $\mathrm{CO}_{2}$ channel with an isotopic standard (checked with IRMS) for in-flight reference to improve isotope ratio precision and accuracy.

$T L S \mathrm{CO}_{2}$ and $\mathrm{H}_{2} \mathrm{O}$ Calibration: Thermal-vacuum test results from the flight TLS instrument showed capability for both $\mathrm{H}_{2} \mathrm{O}$ and $\mathrm{CO}_{2}$ sensitivity at the levels given in Table 10 over a wide range of operating temperatures from $-25^{\circ} \mathrm{C}$ to $44{ }^{\circ} \mathrm{C}$. In the $2 \mathrm{f}$ mode, TLS records the $\mathrm{CO}_{2}$ spectral scan of Fig. 15 with very high signal-to-noise ratio (SNR); about $\sim 40,000: 1$ for moderate amounts of $\mathrm{CO}_{2}$. While high $\mathrm{SNR}$ is achieved for the individual $\mathrm{CO}_{2}$ and water isotope lines, the reproducibility of the ratio of these lines ultimately drives the accuracy of the determined ${ }^{13} \mathrm{C} /{ }^{12} \mathrm{C},{ }^{18} \mathrm{O} /{ }^{17} \mathrm{O} /{ }^{16} \mathrm{O}$ and $\mathrm{D} / \mathrm{H}$ ratios; data from thermal-vacuum testing show that 1-5 per mil is achievable in time scales typical for a SAM experiment in the rover.

SAM soil samples will nominally be about $0.08 \mathrm{~cm}^{3}(>0.1$ gram) for a single sample drop from $\mathrm{SPaH}$ for both EGA and combustion experiments. EGA will produce $\mathrm{CO}_{2}$ at characteristic temperatures from carbonates $\left(\mathrm{FeCO}_{3}, \mathrm{MgCO}_{3}, \mathrm{CaCO}_{3}\right)$ and $\mathrm{H}_{2} \mathrm{O}$ from clays over a wide range of temperatures, and the combustion will oxidize organic carbon to $\mathrm{CO}_{2}$.

With the assumption that Mars samples might contain typically $\sim 0.1$ weight $\%$ of carbonate, $\sim 2$ weight $\%$ of water, and no more than $10^{-3}$ weight $\%$ of organic carbon, the demonstrated sensitivities of TLS given in Table 10 gives an excellent TLS limit of detection equivalent to $\sim 10^{-9}$ wt $\%$ carbonate, and $\sim 10^{-10} \mathrm{wt} \%$ water in solid samples. Much higher carbonate and water content would certainly be expected and these numbers were simply chosen to illustrate the sensitivity of the TLS in SAM for carbonate detection.

TLS $\mathrm{CH}_{4}$ Calibration: Fig. 15 shows actual flight TLS data collected over nearly an hour after the completion of thermal-vacuum environmental testing. The spectral average over this period is shown in the upper panel, showing excellent signal-to-noise for the three parent lines and the four isotopic ${ }^{13} \mathrm{CH}_{4}$ lines. In contrast with the laboratory data of a ${ }^{13} \mathrm{C}$ depleted 
Fig. 23 QMS sensitivity for argon at emission levels of 50 , 100 , and $200 \mu \mathrm{A}$ in (counts/second)/(particle/cc). These experiments were carried out prior to integration of the QMS into the suite

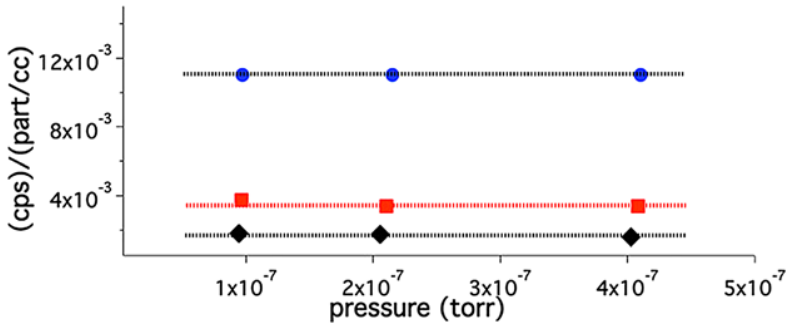

sample, this data is for a sample with ${ }^{13} \mathrm{CH}_{4}$ enriched about four times above its terrestrial value. By integrating over the four isotopic lines and ratioing to the integral over the three parent lines, a single data point (lower panel) is produced for each two-minute spectrum. Scatter about the mean is seen to be a few per mil, and the standard error is $\sim 5$ per mil. The methane concentration in the cell was $\sim 4 \mathrm{ppmv}$, that with a nominal SAM enrichment of one hundred would be equivalent to sampling 40 ppbv on Mars.

During thermal-vacuum testing at JPL, TLS was calibrated over a wide range of gas pressures and cell temperatures using NIST-traceable standards including those of methane at 1800 and 80 ppbv methane. TLS achieved stand-alone methane detection sensitivity across the wide range of cell temperatures from $-25^{\circ} \mathrm{C}$ to $+42{ }^{\circ} \mathrm{C}$ of $0.8 \mathrm{ppb}$ average (Table 10) in a 100 second average, and with data taken for longer integration times, achieved a LOD of $0.3 \mathrm{ppb}$ in 900 seconds (15 minutes). On Mars, longer averaging time will be available, and TLS is expected to achieve $0.1 \mathrm{ppb}$ (100 parts-per-trillion) stand-alone detection sensitivity. We say "expected" because there is still the possibility that the operational conditions on Mars will be different than those of our thermal-vacuum testing at JPL and the NASA GSFC.

As described in Sect. 4.2 above, SAM will provide methane enrichment of at least a factor of 50, with a factor of 100 or more possible (test-bed verification needed). Therefore, based on thermal-vacuum test results using the flight TLS instrument, with an enrichment of 100, TLS in SAM will have capability to detect atmospheric methane abundances of parts-per-trillion in the Martian atmosphere.

Assuming that MSL lands in a region of relatively high methane abundance of $24 \mathrm{ppbv}$, we have used the actual achieved sensitivities to calculate the integration times needed to determine the ${ }^{13} \mathrm{C} /{ }^{12} \mathrm{C}$ isotope ratio in methane to $10 \%$ and the $\mathrm{D} / \mathrm{H}$ ratio in methane to $100 \%$ as shown in Table 10. We note that both measurements will be very difficult without the enrichment process, and that the ${ }^{13} \mathrm{C} /{ }^{12} \mathrm{C}$ isotope ratio should be measurable in a reasonable time with this methane abundance.

GC Calibration: The final qualification of the GC system was in the integrated SAM suite and is described below. Nevertheless, prior to delivery each GC column was calibrated with a gas mixture and the absolute response of the TCD detectors established.

QMS Sensitivity: The sensitivity and linearity of the QMS was established prior to integration into the suite using a variety of gases. For example, Fig. 23 illustrates the sensitivity values derived from a calibration run where argon was introduced into the instrument utilizing a vacuum manifold designed to minimize pressure gradients between the gas in the ion source of the QMS and an ion gauge certified as a National Institute of Standards (NIST) reference. The ${ }^{40} \mathrm{Ar}$ and the ${ }^{36} \mathrm{Ar}$ isotopes at $m / z=40$ and 36 were used to calculate these sensitivity values. The sensitivity is nearly independent of pressure over the density range illustrated. The sensitivity at the highest emission level of $200 \mu \mathrm{A}$ utilized for SAM is somewhat more than double the sensitivity at $100 \mu \mathrm{A}$. This may be due to the changing potential 
Fig. 24 The raw signal $(r)$ and the corrected $(c)$ detector counts per second from the SAM FM are plotted against the manifold pressure for the $m / z=20$ signal in a run where a gas mixture consisting of equal parts of $\mathrm{CO}_{2}$, $\mathrm{N}_{2}, \mathrm{O}_{2}$, and Ar was introduced into the SAM inlet and entered the instrument through the capillary flow restrictor associated with valve V11

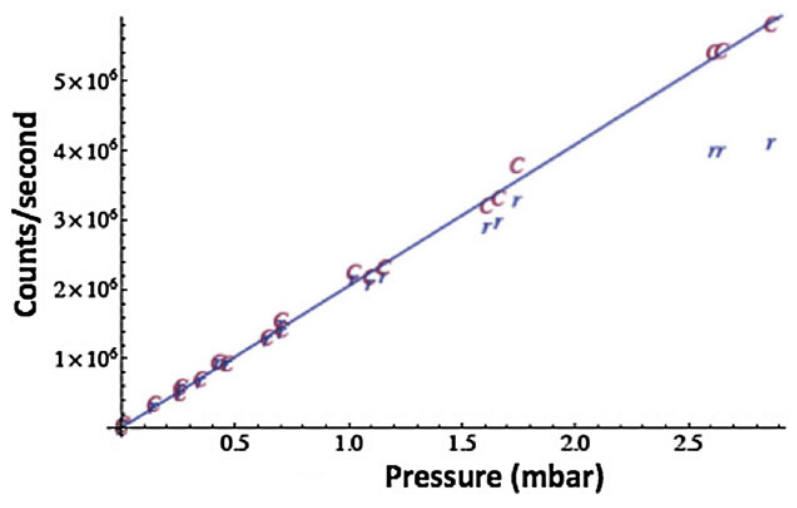

distribution in the ionization region due to space charge effects at these high electron emission currents.

QMS Detector Dead Time Corrections: For single channel electron multipliers operated in a pulse counting mode, there is a minimum length of time that must separate two events for them to be recorded as two separate pulses. This is known as the "dead time" (Knoll 2000). If multiple events occur within the time required for a single output pulse, some events may be lost, leading to erroneously low measurements at high count rates. Accurate quantitative analysis requires a correction to the raw QMS data to account for this phenomenon. A standard form for this correction for paralyzable detector systems is $o=n e^{-\mathrm{n} \tau}$, where $o=$ observed count rate, $n=$ the true count rate, and $\tau=$ the dead time (Knoll 2000). Note that this correction takes the same form as that for "pulse pile-up." Usually $\tau$ is represented by a constant value, typically between 20-80 ns for these types of detectors.

The QMS dead time was first studied on the SAM breadboard (Franz et al. 2011) and determined from calibration of pure $\mathrm{Kr}$ and $\mathrm{SO}_{2}$ by two different methods, which assumed either linearity of the signal at a given $\mathrm{m} / \mathrm{z}$ with pressure or a constant ratio of two isotopes with increasing count rate. Initially it was assumed that the dead time was constant for the system. However, no single value for the dead time could be found that would fit the breadboard calibration data across the full range of pressures and count rates. Instead the mathematical form $\tau=a e^{\mathrm{bo}}$, where $a$ and $b$ are constants and $o=$ observed count rate, was used for the variable dead time correction. Dead times obtained with this formula for $\mathrm{SO}_{2}$, obtained by assuming a constant ratio of $\mathrm{m} / \mathrm{z} 66$ to 64 for the $\mathrm{SO}_{2}$ tank gas, ranged from approximately 2 to $66 \mathrm{~ns}$. This is currently the nominal method for SAM data analysis. The detector begins to saturate at several million counts per second, and the correction is valid only up to the point where the detector output begins to decrease with increasing ion current. Figure 24 illustrates the effect of the correction at these count rates.

QMS Tuning and Mass Calibration: Highly fluorinated synthetic compounds such as perfluorotributylamine $\left(\mathrm{CF}_{3}\left(\mathrm{CF}_{2}\right)_{3}\right)_{3} \mathrm{~N}$ and perfluorophenanthrene $\left(\mathrm{C}_{14} \mathrm{~F}_{24}\right)$ were utilized to tune the QMS and implement temperature compensation algorithms in the software to eliminate or minimize peak shifts with the temperature of the RF circuit. Figure 25 illustrates tuning across the $150 \mathrm{Da}$ boundary between the mid frequency and low frequency RF and the peak shapes in the high frequency portion of the spectrum.

GCMS Data Treatment and SAM Sensitivity: The wide mass range sampled by SAM and the 20 millisecond repeat period for sampling of a new $\mathrm{m} / \mathrm{z}$ value means that a transient such as GC elution of a few seconds duration may be substantially under sampled by the QMS. For this reason the SAM QMS scan algorithm utilizes mass bands to more rapidly sample 

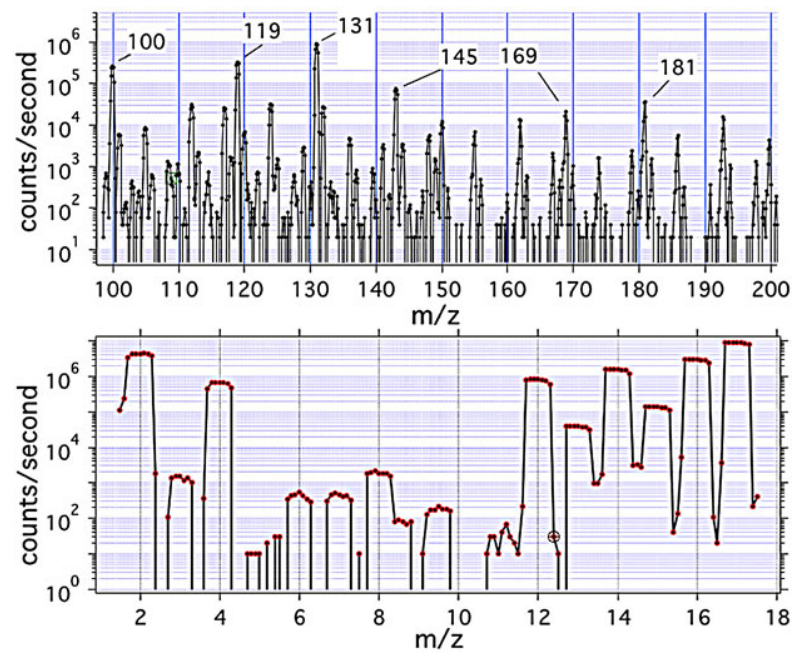

Fig. 25 Peak shapes in the mid and low frequency portions of the FM QMS are illustrated in the top spectrum using PFTBA and in a portion of the high frequency spectrum in the bottom pane. The frequency transition in the top spectrum is at $150 \mathrm{Da}$. Each spectrum is scanned with $0.1 \mathrm{Da}$ mass increments. The flat top peaks enable the unit mass scan modes to be reliably implemented. Note the doubly charged species evident at $m / z=6.0,7.0,8.0,8.5$, and 9.0 from $\mathrm{C}^{++}, \mathrm{N}^{++}, \mathrm{O}^{++}, \mathrm{OH}^{++}$, and $\mathrm{H}_{2} \mathrm{O}^{++}$respectively. Comparison of the counts at $2 \mathrm{Da}$ with the zero counts at $3 \mathrm{Da}$ demonstrates that the crosstalk between adjacent unit mass values for the high frequency portion of the spectrum is greater than 6 orders of magnitude

a wide $m / z$ space with higher time resolution. The bandwidth for a particular mass range of interest can be set by selection of the DAC's that control the RF and DC potentials. All ions within that mass delta are then transmitted through the quadrupole to the detector and counted in a single integration period. A useful bandwidth for providing robust sampling of GCMS peak shapes has been found to be $\Delta m=25 \mathrm{Da}$. During a GCMS run each band is also associated with a threshold and a priority. If the signal from that IP is above the threshold then the unit $m / z$ values in that mass region are sampled as soon as the priority for that band allows. To enable robust sampling at every $m / z$ value the band peak shapes during a GCMS run are used to fit any under-sampled individual unit $m / z$ values. This scheme presently implemented in the SAM flight software allows accurate fragmentation patterns for identification of a rapidly eluting organic compound to be established (Fig. 26). For typical general purpose, scans employing these band algorithms over the full mass range a typical sensitivity limit for GCMS analysis (Fig. 27) was found to be $\sim 10^{-12}$ moles. For a typical single volume of sample delivered $(0.078 \mathrm{cc})$ this sensitivity would translate to $\sim 0.6 \mathrm{ppb}$ by mass for an assumed sample density of $2 \mathrm{~g} / \mathrm{m}$ and a molecular weight of organic of $100 \mathrm{Da}$. Delivery of several aliquots of sample into the SAM cup or a focused search for a particular set of molecules using a more limited QMS mass scan could provide improved sensitivity. Figure 28 illustrates chromatograms from both the TCD and QMS detectors.

Static Mass Spectrometry and Trace Noble Gas Measurements: A sufficient set of calibration experiments were carried out on the SAM FM to validate the performance of the QMS in static mode for the trace noble gas measurements. In the example data shown in Fig. 29 xenon was introduced into the QMS to the levels expected for atmospheric mixing ratios of tens of ppbv in a AS-NG sequence after SAM GPS processing. This processing in a AS-NG sequence consists of removal of the chemically active species through the use of 

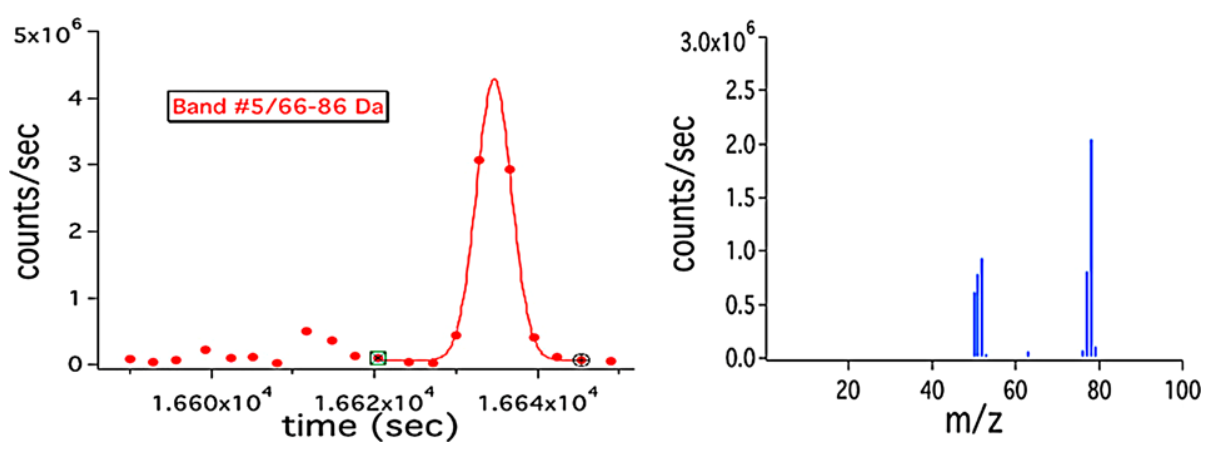

Fig. 26 The band plot in the upper left is fit between the markers with a Gaussian shape peak. Even though in this experiment each benzene fragment is only sampled once during the elution of this molecule from the GC column, the benzene fragmentation pattern (right) can be reconstructed by utilizing the peak shape established by the band where all $\mathrm{m} / z$ values in the 66-86 Da were simultaneously sampled. GC TCD signals can be used to further refine the peak shapes established by the band fits

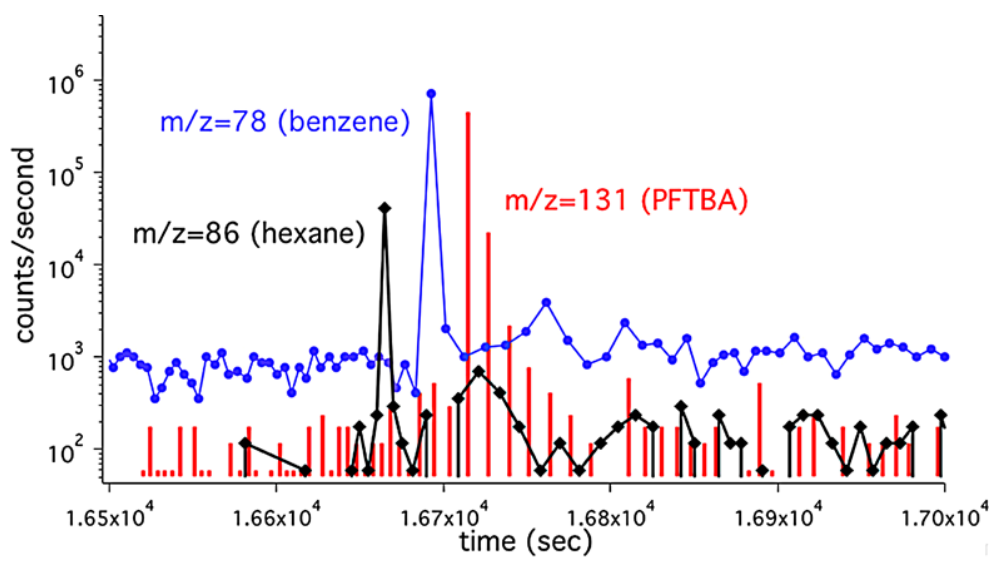

Fig. 27 SAM FM GCMS data acquired during calibration shows S/N ratios of $\sim 1000$ to $\sim 10,000$ from the selected single ion chromatograms depending on the instrument background. The fractional ion signal in the fragment selected is $0.036,0.53$, and 0.48 for hexane, benzene, and PFTBA respectively. In this example, the instrument exhibits a higher background signal at the $m / z=78$ channel from benzene from previous calibration runs. With $\sim 3.7$ nanomoles of benzene and hexane processed in this run the detection limit derived from just these chromatograms is in the picomole range

the scrubbers and getters, trapping of the krypton and xenon on the SAM HC trap, pumping away the light noble gases after their measurement by the QMS in dynamic mode, followed by full transport of the krypton and xenon trapped on the SAM HC trap to the QMS using V11 where static QMS measurements are made with HCV1 closed using only the getter G1 for pumping of residual active gases in the QMS. The precision for the isotopes shown in this figure is better than $0.5 \%$.

\subsection{Upcoming SAM Characterization with the SAM Testbed}

In order to keep the SAM FM as free from terrestrial contamination as possible and to restrict use of consumables such as the onboard calibrants, helium, and the pump bearings, 


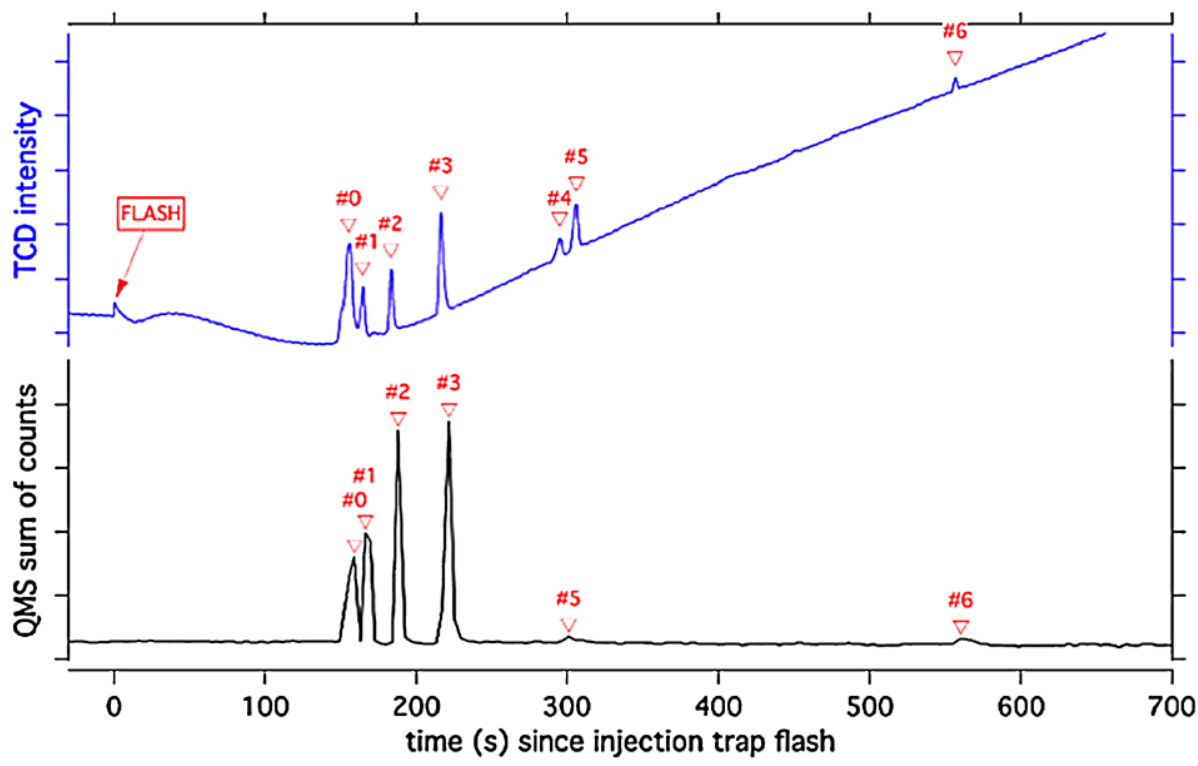

Fig. 28 Chromatograms obtained with the SAM GC instrument for the analysis of a gaseous mixture of hydrocarbons (butane, pentane, hexane and benzene diluted at $1000 \mathrm{ppmv}$ in helium) and PFTBA to provide nano-moles of these compounds on the SAM trap before the GCMS run. The GC5 analytical channel was used. Both TCD and MS signal are plotted, respectively in the upper and lower part of the figure. The QMS signal corresponds to the total ion current measured with the MS, obtained with the 5-250 m/q range. GC operating conditions: column temperature: $50{ }^{\circ} \mathrm{C}$ at $t=0 \mathrm{sec}$ then heated at $10{ }^{\circ} \mathrm{C} / \mathrm{min}$ rate up to $250{ }^{\circ} \mathrm{C}$ for $1 \mathrm{~min}$; TCD temperature $85^{\circ} \mathrm{C}$; IT heated up to approximately $250{ }^{\circ} \mathrm{C}$ for $5 \mathrm{sec}$ at $t=0 \mathrm{sec}$. Peaks identification: 0 : butane, 1 : pentane, 2 : hexane, 3 : benzene, $4,5,6$ : background compounds

only a limited number of well-characterized samples were introduced to SAM during qualification and calibration. Nevertheless, these provided a good measure of the instrument performance, resource requirements, and operational constraints. While breadboard instruments have provided valuable data on pure minerals and terrestrial analog samples, an even higher fidelity measure of the SAM performance will come with the operation of the SAM Testbed (TB). The SAM TB will be operated in the environmental chamber designed for test, qualification, and calibration of the SAM FM. Its completion and initial operation is scheduled for well before MSL landing on August 6, 2012 so that certain sequences that were not run on the SAM FM for contamination or consumable considerations could be vetted prior to MSL landing or during the early part of its landed mission. An important example of this is the wet chemical processing experiments described in Sect. 4.6. The SAM TB will also serve to test new scripts where significant changes have been made in order to increase the probability of robust operation of a new script uploaded to the Curiosity rover for execution by SAM.

\subsection{SAM Archival Data Products}

SAM data products will be archived in the Geosciences Node of the Planetary Data System. The raw SAM data files will be delivered to the SAM team as Experiment Data Record (EDR) products generated by the Multi-mission Image Processing Laboratory (MIPL) at JPL under the Operations Products Generation Subsystem (OPGS), using the telemetry processing software MSLEdrGen. These products are created by MIPL during operations from: 
Fig. 29 Static mode performance of the SAM FM QMS is demonstrated in this calibration experiment where xenon was introduced into the mass spectrometer to a level expected from the present estimate of its atmospheric mixing ratio. In the static mode only the passive getter pump is utilized to remove instrument background gases and the valve to the turbomolecular pump is closed. The entire set of measurements that generated the ratio plot in the bottom frame was acquired in 30 seconds. More extensive testing of this AT-NG sequence is planned utilizing the SAM testbed

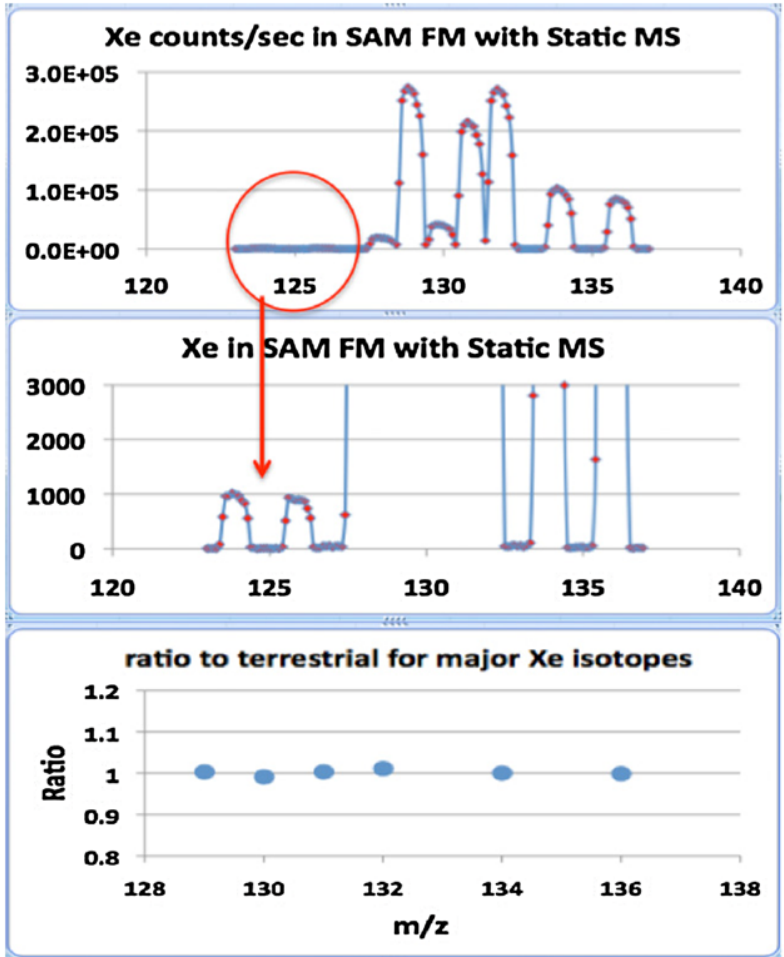

(a) Mission Data Processing and Control System (MPCS) data products, (b) SPICE kernels, and (c) a meta-data database. They are created on the MSL Operations Data Store (ODS) and then deposited into MIPL's File Exchange Interface (FEI) for electronic distribution to remote sites/users via a secure subscription protocol. The EDR product is considered NASA packet-level data. SAM EDR data products will be stored at the PDS for safekeeping, but will not be formally archived in PDS. The SAM team will convert raw data to a more manageable format before delivery to the PDS for archiving as the lowest-level RDR product, which is considered to be NASA level 0 data. Higher-level RDR products will represent the results of further data manipulation and interpretation. The planned SAM data products are shown in Table 13.

\section{Summary}

SAM is designed to enable a range of contributions to Mars science. These include studies of atmospheric chemistry with measurements of diurnal and seasonal changes in the atmospheric composition. An improved determination of noble gases abundances and isotope ratios in the atmosphere can ground models of atmospheric evolution. The SAM measurements should clarify the outstanding question of the presence or absence of methane in the atmosphere of Mars and its variability on a seasonal basis. The comparison of isotopes of noble gases plus $\mathrm{C}, \mathrm{H}, \mathrm{N}, \mathrm{S}$, and $\mathrm{O}$ in atmospheric gases and in noble gases and simple molecules evolved from a diverse set of solid samples in the floor and the layered mounds of Gale crater can provide insight into reservoirs of volatiles and processes that have shaped 
Table 13 SAM data products

Product NASA Description

PDS data set ID

name level

\begin{tabular}{|c|c|c|c|}
\hline SAM LO & 0 & $\begin{array}{l}\text { Unpacking of telemetry into data numbers as raw ADC values or } \\
\text { counts and verification of data integrity }\end{array}$ & $\begin{array}{l}\text { MSL-M-SAM- } \\
\text { 2-RDR-V1.0 }\end{array}$ \\
\hline $\begin{array}{l}\text { SAM } \\
\text { L1A }\end{array}$ & $1 \mathrm{~A}$ & Conversion of raw ADC values or counts to science units & $\begin{array}{l}\text { MSL-M-SAM- } \\
\text { 3-RDR-V1.0 }\end{array}$ \\
\hline $\begin{array}{l}\text { SAM } \\
\text { L1B }\end{array}$ & $1 \mathrm{~B}$ & $\begin{array}{l}\text { Application of corrections to data, e.g., detector dead time, TCD } \\
\text { temperature, noise removal, corrections for saturation and instrument } \\
\text { response function. Instrument-specific data may include the following } \\
\text { types of information: } \\
\text { QMS: time, } m / z \text {, signal, sorted by gas inlet } \\
\text { EGA: oven temperature, } m / z \text {, signal } \\
\text { GC: TCD signal vs. retention time; pressure; temperature; column used } \\
\text { GCMS: time, mass spectra data; smart scanning data } \\
\text { TLS: direct and harmonic spectra }\end{array}$ & $\begin{array}{l}\text { MSL-M-SAM- } \\
\text { 4-RDR-V1.0 }\end{array}$ \\
\hline SAM L2 & 2 & $\begin{array}{l}\text { Tables and reportsdescribing data interpretation as completed by the } \\
\text { SAM science team. Further data correction will include background } \\
\text { subtraction. Examples of instrument-specific data include: } \\
\text { QMS: gas composition; isotope ratios } \\
\text { EGA: gas composition vs. sample temperature } \\
\text { GC: species; relative abundance } \\
\text { GCMS: identification of EGA products and derivatized compounds } \\
\text { TLS: abundance and isotope ratios }\end{array}$ & $\begin{array}{l}\text { MSL-M-SAM- } \\
\text { 5-RDR-V1.0 }\end{array}$ \\
\hline
\end{tabular}

Mars since the formation of this crater more than 3 billion years ago. The controlled evolved gas analysis of solid samples delivered to SAM complements other Curiosity rover instruments and facilitates the analysis of the chemical state and the identification of mineral phases. These measurements in combination with the full MSL science payload address the duration and extent of wetter surface environments and the chemical state and potential habitability of the surface environment on ancient Mars. The combination of SAM's three instrument types with integrated gas and solid sample manipulation subsystems, enables efficiency in processing and allows multiple measurement techniques to be applied to a single sample in a well-coordinated manner.

A core goal of the SAM investigation is to provide a broad survey for organic molecules in the ancient layers of the mound of Gale crater, the first such exploration since the Viking mission more than 3 decades ago. The absence or presence of organic compounds will contribute to our understanding of the effects of the chemical and radiation environments in near-surface materials at sites like Gale crater. The characteristics of terrestrial organic compounds are dramatically shaped in nearly every surface and near-surface environment by biological processes so the in situ discovery of organic compounds in the rocks of Gale crater would motivate a new set of astrobiology studies to explore their sources and processing on this and future missions. Early steps in these more detailed studies would use the wet chemistry GCMS capabilities of SAM to search for molecules of astrobiological relevance.

The determination of habitability potential on Mars is clearly made difficult in the absence of obvious current life, and the environment on Earth is so overprinted with the signatures of life, it is a difficult benchmark against which to compare the martian indicators of past and present habitability. There are tantalizing clues about the former chemical potential of Mars in the SNC meteorites. Substantial advances have been made in the past decade in understanding the extent of surface aqueous processing and mineralogical diversity on Mars 
from surface landers and rovers and from orbital measurements. These meteorite, orbital, and in situ analyses from diverse locations on Mars and the focused orbital studies of the Gale Crater mound layers provide both mineralogical and morphological clues and context that allow us to form hypotheses about the surface processes that emplaced them. These hypotheses, followed by the variety of chemical, isotopic, mineralogical, morphological, and textural measurements to be made by MSL instruments will enable us to evaluate the habitability potential of Mars through time with a greater number of parameters than the presence or absence of the signatures of liquid water. We have successfully followed the water, and the SAM scientific investigation is ready to conduct its varied set of chemical and isotopic composition measurements together with the rest of the MSL science investigations to learn if other features of habitable environments have ever existed on Mars.

Acknowledgements Support from the Mars Science Laboratory Payload Manager Jeff Simmonds and the Project Science Team John Grotzinger, Joy Crisp, and Ashwin Vasvada, the Program Scientist Michael Meyer, and the first Project Scientist Edward Stolper is acknowledged. From the French Space Agency CNES A. Gaboriaud for CNES CST engineering and for the CNES ground segment Eric Lorigny. Contributions to the SAM science definition are acknowledged from Wes Huntress, Robert Pepin, Wayne Kasprzak, Fred Goesmann, Wadhwa Meenakshi, Edward Vicenzi, Kenneth Nealson, Audrey Noblet, Carole Philippon, Loic Soldiani, Alain Person, Claude Geffroy-Rodier, and Guy Israel. Special acknowledgement is given SAM Coinvestigator James Scott and science collaborator Gordon McKay who both passed away during the SAM development. Helpful contributions to analog studies were contributed by Brad Sutter and Doug Archer. In addition to co-authors, numerous individuals contributed to the design, fabrication, qualification, integration, test, and calibration of SAM. These include James Odom, Irving Linares, Phillip Goodman, Mark Wolf, Curt Cooper, Ray Bendt, Steven Cagiano, Yury Flom, Suong Le, John Bishop, Mark McClendon, Roger Counts, Keith Corse, Joyce Manning, Greg Hidrobo, Ryan Wilkinson, Rick Scott, Matthew Lefavor, George Winkert, Norman Dobson, Nick Dobson, Mathew Lefavor, David Mcclaeb, Jerry Hengemihle, Kiran Patel, Micah Johnson, Christa Budinoff, Ferzan Jaeger, Joelle Cooperrider, Akif Ersahin, Charles Fleetwood, John Canham, Rebecca Prats, Robert Kiwak, Anthony Sanders, Jamie Demick, Carol Lilly, Lou Fetter, Bruce Meinhold, Christine Collins, Francesco Giacobbe, Amil Mann, Mario Martins, Shawn McLeod, Carol Mosier, Daniel Powers, Brian Rice, Cynthis Simmons, Ray Trunzo, Janelle Vorreiter, Kiel Davis, Jerri Ji, Lee Carlson, Thomas Kennedy, Michael Rutberg, Marie-Sophie Clerc, Jean-Jacques Correia, Alexandre Galic, Vincent Guerrini, Mustapha Meftah, Christophe Montaron, Patrick Poinsignon, Curt Henry, Martin Buehler, Steve Woodward, and Jean-Baptiste Rigal. Special acknowledgement is given a valued Goddard colleague Robert Abell who contributed greatly to the fabrication of critical SAM elements before his death in 2008. During SAM integration with Curiosity Rover significant support was provided by Richard Redick and Joseph Melko of JPL. SAM-GC industrial partners included Air Liquide Advanced Technologies, Comat Aerospace, ATERMES, Restek, and Varian Inc. (now Agilent Technologies). Thanks to Ray Ardvison and Paul Niles for helpful reviewer comments. Finally support from many individuals at NASA Headquarters and both Goddard and JPL management is acknowledged with special thanks to Orlando Figueroa, Charles Elachi, Doug McCuistion, Jim Garvin, Anne Kinney, and Ed Weiler. The SAM GC was funded by the Centre National d'Etudes Spaciales and the SAM Suite by the Science Mission Directorate of the National Aeronautics and Space Administration.

Open Access This article is distributed under the terms of the Creative Commons Attribution License which permits any use, distribution, and reproduction in any medium, provided the original author(s) and the source are credited.

\section{References}

M.H. Acuna, J.E.P. Connerney, N.F. Ness, R.P. Lin, D. Mitchell, C.W. Carlson, P. Cloutier, Global distribution of crustal magnetization discovered by the Mars Global Surveyor MAG/ER experiment. Science 284(5415), 790-793 (1999)

M. Anpo, K. Chiba, Photocatalytic reduction of $\mathrm{CO}_{2}$ on anchored titanium-oxide catalysts. J. Mol. Catal. A, Chem. 74(1-3), 207-212 (1992)

S.K. Atreya, Z.G. Gu, Stability of the martian atmosphere-is heterogeneous catalysis essential. J. Geophys. Res. 99(E6), 13133-13145 (1994) 
S.K. Atreya, P.R. Mahaffy, A.S. Wong, Methane and related trace species on Mars: origin, loss, implications for life, and habitability. Planet. Space Sci. 55(3), 358-369 (2007)

S.K. Atreya, A.S. Wong, N.O. Renno, W.M. Farrell, G.T. Delory, D.D. Sentman, D.C. Catling, Oxidant enhancement in martian dust devils and storms: implications for life and habitability. Astrobiology 6(3), 439-450 (2006)

A. Banin, L. Margulies, Simulation of viking biology experiments suggests smectites not palagonites, as martian soil analogs. Nature 305(5934), 523-525 (1983)

L. Becker, D.P. Glavin, J.L. Bada, Polycyclic aromatic hydrocarbons (PAHs) in Antarctic martian meteorites, carbonaceous chondrites, and polar ice. Geochim. Cosmochim. Acta 61, 475-481 (1997)

L. Becker, B. Popp, T. Rust, J.L. Bada, The origin of organic matter in the martian meteorite ALH84001. Earth Planet. Sci. Lett. 167, 71-79 (1999)

S.A. Benner, K.G. Devine, L.N. Matveeva, D.H. Powell, The missing organic molecules on Mars. Proc. Natl. Acad. Sci. USA 97, 2425-2430 (2000)

J.L.Y. Bibring, A. Gendrin, B. Gondet, F. Oulet, M. Berthe, A. Soufflot, R. Arvidson, N. Mangold, J. Mustard, P. Drossart, The OMEGA Team, Mars surface diversity as revealed by the OMEGA/Mars Express observations. Science 307, 1576-1581 (2006a)

J.P. Bibring, Y. Langevin, J.F. Mustard, F. Poulet, R. Arvidson, A. Gendrin, O. Team, Global mineralogical and aqueous mars history derived from OMEGA/Mars express data. Science 312(5772), 400-404 (2006b)

J.P. Bibring, S.W. Squyres, R.E. Arvidson, Merging views on Mars. Science 313(5795), 1899-1901 (2006c)

K. Biemann, J. Oro, P. Toulmin III, L.E. Orgel, A.O. Nier, D.M. Anderson, P.G. Simmonds, Search for organic and volatile inorganic compounds in two surface samples from the Chryse Planitia region of Mars. Science 194, 72-76 (1976)

K. Biemann, J. Oro, P. Toulmin III, L.E. Orgel, A.O. Nier, D.M. Anderson, P.G. Simmonds, The search for organic substances and inorganic volatile compounds in the surface of Mars. J. Geophys. Res. 82, 4641-4658 (1977)

J.L. Bishop, R.T. Schelble, C.P. McKay, A.J. Brown, K.A. Perry, Carbonate rocks in the Mojave Desert as an analogue for martian carbonates. Int. J. Astrobiol. 10, 349-358 (2011)

T.R. Blackburn, H.D. Holland, G.P. Ceasar, Viking gas exchange reaction: simulation on UV-irradiated manganese dioxide substrate. J. Geophys. Res. 84, 8391-8394 (1979)

D.L. Blaney, T.B. McCord, Indications of sulfate minerals in the martian soil from Earth-based spectroscopy. J. Geophys. Res. 100, 14433-14442 (1995)

D. Bogard, A reappraisal of the martian ${ }^{36} \mathrm{Ar} /{ }^{38} \mathrm{Ar}$ ratio. J. Geophys. Res. 102, 1653-1661 (1997)

D.D. Bogard, R.N. Clayton, K. Marti, T. Owen, G. Turner, Martian volatiles: isotopic composition, origin, and evolution. Space Sci. Rev. 96, 425-458 (2001)

O. Botta, J.L. Bada, Extraterrestrial organic compounds in meteorites. Surv. Geophys. 23(5), 411-467 (2002)

O. Botta, D.P. Glavin, J.L. Bada, Identification of amino acid signatures in carbonaceous chondrites. Meteorit. Planet. Sci. Suppl. 36, 26 (2001)

A. Bouvier, J. Blichert-Toft, J.D. Vervoort, F. Albarede, The age of SNC meteorites and the antiquity of the martian surface. Earth Planet. Sci. Lett. 240(2), 221-233 (2005)

W.V. Boynton, D.W. Ming, S.P. Kounaves, S.M.M. Young, R.E. Arvidson, M.H. Hecht, R.V. Morris, Evidence for calcium carbonate at the Mars Phoenix landing site. Science 325(5936), 61-64 (2009)

J.C. Bridges, M.M. Grady, Evaporite mineral assemblages in the nakhlite (martian) meteorites. Earth Planet. Sci. Lett. 176, 267-279 (2000)

J.C. Bridges, P.H. Warren, The SNC meteorites: basaltic igneous processes on Mars. J. Geol. Soc. 163, 229-251 (2006)

J.C. Bridges, D.C. Catling, J.M. Saxton, T.D. Swindle, I.C. Lyon, M.M. Grady, Alteration assemblages in martian meteorites: implications for near-surface processes. Space Sci. Rev. 96, 365-392 (2001)

A. Buch, R. Sternberg, C. Szopa, C. Freissinet, C. Garnier, E.J. Bekri, P.R. Mahaffy, Development of a gas chromatography compatible Sample Processing System (SPS) for the in-situ analysis of refractory organic matter in martian soil: preliminary results. Adv. Space Res. 43, 143-151 (2009)

M. Cabane, P. Coll, G. Israël, F. Raulin, H. Niemann, P. Mahaffy, W. Brinckerhoff, Organic and inorganic signatures in Mars ground and underground, one of the goals for "SAM" (Sample Analysis at Mars), in Exo-Astrobiology, vol. 518, ed. by H. Lacoste (2002), pp. 323-326

M.P. Callahan, K.E. Smith, H.J. Cleaves II, J. Ruzicka, J.C. Stern, D.P. Glavin, C.H. House, J.P. Dworkin, Carbonaceous meteorites contain a wide range of extraterrestrial nucleobases. Proc. Natl. Acad. Sci. USA 108, 13995-13998 (2011)

M.H. Carr, J.W. Head, Geologic history of Mars. Earth Planet. Sci. Lett. 294(3-4), 185-203 (2010)

D.C. Catling, M.W. Claire, K.J. Zahnle, R.C. Quinn, B.C. Clark, M.H. Hecht, S. Kounaves, Atmospheric origins of perchlorate on Mars and in the Atacama. J. Geophys. Res. 115 (2010) 
C. Chyba, C. Sagan, Endogenous production, exogenous delivery and impact-shock synthesis of organic molecules: an inventory for the origins of life. Nature 355, 125-132 (1992)

R.T. Clancy, B.J. Sandor, G.H. Moriarty-Schieven, A measurement of the $362 \mathrm{GHz}$ absorption line of Mars atmospheric $\mathrm{H}_{2} \mathrm{O}_{2}$. Icarus 168, 116-121 (2004)

R.T. Clancy, D.O. Muhleman, B.M. Jakosky, Variability of carbon monoxide in the Mars atmosphere. Icarus 55(2), 282-301 (1983)

B.C. Clark, R.V. Morris, S.M. McLennan, R. Gellert, B. Jolliff, A.H. Knoll, R. Rieder, Chemistry and mineralogy of outcrops at Meridiani Planum. Earth Planet. Sci. Lett. 240, 73-94 (2005)

B.C. Clark, D.C. van Hart, The salts of Mars. Icarus 45, 370-378 (1981)

C.S. Cockell, A.C. Schuerger, D. Billi, E. Imre Friedmann, C. Panitz, Effects of a simulated martian UV flux on the Cyanobacterium, Chroococcidiopsis sp. 029. Astrobiology 5, 127-140 (2005)

J. Crovisier, W.M. Irvine, T. Owen, L. Becker, J. Blank, J.R. Brucato, F. Robert, The molecular complexity of Comets, in Astrobiology: Future Perspectives, vol. 305, ed. by P. Ehrenfreund (2004), p. 179

L.R. Dartnell, L. Desorgher, J.M. Ward, A.J. Coates, Modelling the surface and subsurface martian radiation environment: implications for astrobiology. Geophys. Res. Lett. 34, 02207 (2007)

L.R. Dartnell, L. Desorgher, J.M. Ward, A.J. Coates, Modelling the surface and subsurface martian radiation environment: implications for astrobiology. Int. J. Astrobiol. 7(1), 64 (2008)

T.A. Delchar, Vacuum Physics and Techniques (Chapman \& Hall, London, 1993)

G.T. Delory, W.M. Farrell, S.K. Atreya, N.O. Renno, A.S. Wong, S.A. Cummer, D.C. Catling, Oxidant enhancement in martian dust devils and storms: storm electric fields and electron dissociative attachment. Astrobiology 6(3), 451-462 (2006)

B.L. Ehlmann, J.F. Mustard, S.L. Murchie, F. Poulet, J.L. Bishop, A.J. Brown, J.J. Wray, Orbital identification of carbonate-bearing rocks on Mars. Science 322, 1828 (2008)

P. Ehrenfreund, S.B. Charnley, Organic molecules in the interstellar medium, comets, and meteorites: a voyage from dark clouds to the early Earth. Annu. Rev. Astron. Astrophys. 38, 427-483 (2000)

J.L. Eigenbrode, Fossil lipids for life-detection: a case study from the early Earth record. Space Sci. Rev. 135, 161-185 (2008)

T. Encrenaz, B. Bezard, T.K. Greathouse, J.H. Lacy, M.J. Richter, S.K. Atreya, A.S. Wong, Mars. Int. Astron. Union Circ. 8254, 2 (2003)

T. Encrenaz, B. Bezard, T.K. Greathouse, M.J. Richter, J.H. Lacy, S.K. Atreya, F. Forget, Hydrogen peroxide on Mars: evidence for spatial and seasonal variations. Icarus 170, 424-429 (2004)

T. Encrenaz, T.K. Greathouse, M.J. Richter, B. Bezard, T. Fouchet, E. Millour, A search for $\mathrm{SO}_{2}$ on Mars from infrared spectroscopy, in Mars Atmosphere: Modelling and Observation, ed. by F. Forget (2011a), pp. 315

T. Encrenaz, T.K. Greathouse, M.J. Richter, J.H. Lacy, T. Fouchet, B. Bezard, S.K. Atreya, A stringent upper limit to $\mathrm{SO}_{2}$ in the martian atmosphere. Astron. Astrophys. 530, 37 (2011b)

J. Farquhar, J. Savarino, T.L. Jackson, M.H. Thiemens, Evidence of atmospheric sulphur in the martian regolith from sulphur isotopes in meteorites. Nature 404(6773), 50-52 (2000)

K. Fast, T. Kostiuk, F. Espenak, J. Annen, D. Buhl, T. Hewagama, F. Schmülling, Ozone abundance on Mars from infrared heterodyne spectra. I. Acquisition, retrieval, and anticorrelation with water vapor. Icarus 181, 419-431 (2006a)

K. Fast, T. Kostiuk, T. Hewagama, M.F. A'Hearn, T.A. Livengood, S. Lebonnois, F. Lefèvre, Ozone abundance on Mars from infrared heterodyne spectra. II. Validating photochemical models. Icarus 183, 396402 (2006b)

G.J. Flynn, The delivery of organic matter from asteroids and comets to the early surface of Mars. Earth Moon Planets 72, 469-474 (1996)

V. Formisano, S. Atreya, T. Encrenaz, N. Ignatiev, M. Giuranna, Detection of methane in the atmosphere of Mars. Science 306(5702), 1758-1761 (2004)

J.L. Fox, The production and escape of nitrogen atoms on Mars. J. Geophys. Res. 98, 3297-3310 (1993)

J.L. Fox, A. Dalgarno, The production of nitrogen atoms on Mars and their escape. Planet. Space Sci. 28, 41-46 (1980)

H.B. Franz, P.R. Mahaffy, W. Kasprzak, E. Lyness, E. Raaen, Measuring sulfur isotope ratios from solid samples with the sample analysis at Mars instrument and the effects of dead time corrections, in Lunar and Planetary Institute Science Conference Abstracts, vol. 42 (2011), p. 2800

D.H. Garrison, D.D. Bogard, Isotopic composition of trapped and cosmogenic noble gases in several martian meteorites. Meteorit. Planet. Sci. 33, 721-736 (1998)

C. Geffroy-Rodier, L. Grasset, R. Sternberg, A. Buch, A. Ambles, Thermochemolysis in search for organics in extraterrestrial environments. J. Anal. Appl. Pyrolysis 85(1-2), 454-459 (2009)

A. Geminale, V. Formisano, G. Sindoni, Mapping methane in martian atmosphere with PFS-MEX data. Planet. Space Sci. 59(2-3), 137-148 (2011) 
C.D. Gillmann, P. Lognonné, E. Chassefière, M. Moreira, The present-day atmosphere of Mars: where does it come from? Earth Planet. Sci. Lett. 277, 384-393 (2009)

F. Goesmann, H. Rosenbauer, R. Roll, C. Szopa, F. Raulin, R. Sternberg, G. Munoz-Caro, Cosac, the cometary sampling and composition experiment on philae. Space Sci. Rev. 128, 257-280 (2007)

M.P. Golombek, J.A. Grant, L.S. Crumpler, R. Greeley, R.E. Arvidson, J.F. Bell, S.W. Squyres, Erosion rates at the Mars Exploration Rover landing sites and long-term climate change on Mars. J. Geophys. Res. 111, E12 (2006)

M.M. Grady, I.P. Wright, C.T. Pillinger, A carbon and nitrogen isotope study of Zagami. J. Geophys. Res. 102, 9165-9173 (1997)

M.M. Grady, A.B. Verchovsky, I.P. Wright, Magmatic carbon in martian meteorites: attempts to constrain the carbon cycle on Mars. Int. J. Astrobiol. 3, 117-124 (2004)

J.P. Greenwood, Modified sulfur isotopic compositions of sulfides in the Nakhlites and Chassigny. Geochim. Cosmochim. Acta 64, 1121-1131 (2000)

M.H. Hecht, S.P. Kounaves, R.C. Quinn, S.J. West, S.M.M. Young, D.W. Ming, P.H. Smith, Detection of perchlorate and the soluble chemistry of martian soil at the phoenix lander site. Science 325(5936), 64-67 (2009)

M.M. Hirschmann, A.C. Withers, Ventilation of $\mathrm{CO}_{2}$ from a reduced mantle and consequences for the early martian greenhouse. Earth Planet. Sci. Lett. 270, 147-155 (2008)

R.L. Huguenin, K.J. Miller, W.S. Harwood, Frost-weathering on Mars-experimental evidence for peroxide formation. J. Mol. Evol. 14, 103-132 (1979)

J.A. Hurowitz, N.J. Tosca, S.M. McLennan, M.A.A. Schoonen, E. Stansbery, Mechanically produced radical species at silicate surfaces and the oxidant in martian soils, in 36th Annual Lunar and Planetary Science Conference, vol. 36, ed. by S. Mackwell (2005), p. 1991

B.M. Jakosky, R.O. Pepin, R.E. Johnson, J.L. Fox, Mars atmospheric loss and isotopic fractionation by solarwind-induced sputtering and photochemical escape. Icarus 111, 271-288 (1994)

B.M. Jakosky, A.P. Zent, R.W. Zurek, The Mars water cycle: determining the role of exchange with the regolith. Icarus 130(1), 87-95 (1997)

A.J.T. Jull, J.W. Beck, C. Courtney, D.A. Jeffrey, Carbon isotopic evidence for terrestrial organic compounds found in some martian meteorites, in Lunar and Planetary Institute Science Conference Abstracts, vol. 29 (1998a), p. 1184

A.J.T. Jull, C. Courtney, D.A. Jeffrey, J.W. Beck, Isotopic evidence for a terrestrial source of organic compounds found in martian meteorites Allan Hills 84001 and Elephant Moraine 79001. Science 279, 366 (1998b)

E. Kaal, H.G. Janssen, Extending the molecular application range of gas chromatography. J. Chromatogr. A 1184(1-2), 43-60 (2008)

H.R. Karlsson, R.N. Clayton, E.K. Gibson Jr., T.K. Mayeda, Water in SNC meteorites-evidence for a martian hydrosphere. Science 255, 1409-1411 (1992)

W. Kasprzak, H. Niemann, D. Harpold, J. Richards, H. Manning, E. Patrick, P. Mahaffy, Cassini orbiter ion and neutral mass spectrometer instrument, in Society of Photo-optical Instrumentation Engineers (SPIE) Conference Series, vol. 2803, ed. by L. Horn (1996), pp. 129-140

H.P. Klein, N.H. Horowitz, G.V. Levin, V.I. Oyama, J. Lederberg, A. Rich, R.D. Johnson, Viking biological investigation-preliminary-results. Science 194(4260), 99-105 (1976)

G. Klingelhofer, R.V. Morris, B. Bernhardt, C. Schroder, D.S. Rodionov, P.A. de Souza, R.E. Arvidson, Jarosite and hematite at Meridiani Planum from Opportunity's Mossbauer spectrometer. Science 306(5702), 1740-1745 (2004)

G. Kminek, J.L. Bada, The effect of ionizing radiation on the preservation of amino acids on Mars. Earth Planet. Sci. Lett. 245(1-2), 1-5 (2006)

G.F. Knoll, Radiation Detection and Measurement (Wiley, New York, 2000)

M. Komiya, A. Shimoyama, Organic compounds from insoluble organic matter isolated from the Murchison carbonaceous chondrite by heating experiments. Bull. Chem. Soc. Jpn. 69(1), 53-58 (1996)

S.P. Kounaves, M.H. Hecht, J. Kapit, R.C. Quinn, D.C. Catling, B.C. Clark, J. Shusterman, Soluble sulfate in the martian soil at the Phoenix landing site. Geophys. Res. Lett. 37, 09201 (2010)

V.A. Krasnopolsky, Photochemistry of the martian atmosphere (mean conditions). Icarus 101, 313-332 (1993)

V.A. Krasnopolsky, P.D. Feldman, First detection of molecular hydrogen in the atmosphere of Mars: implications for evolution of water. Bull. Am. Astron. Soc. 33, 1122 (2001)

V.A. Krasnopolsky, G.L. Bjoraker, M.J. Mumma, D.E. Jennings, High-resolution spectroscopy of Mars at 3.7 and $8 \mu \mathrm{m}$ : a sensitive search for $\mathrm{H}_{2} \mathrm{O}_{2}, \mathrm{H}_{2} \mathrm{CO}, \mathrm{HCl}$, and $\mathrm{CH}_{4}$, and detection of HDO. J. Geophys. Res. 102(E3), 6525-6534 (1997)

V.A. Krasnopolsky, J.P. Maillard, T.C. Owen, Detection of methane in the martian atmosphere: evidence for life? Icarus 172(2), 537-547 (2004) 
V.A. Krasnopolsky, J.P. Maillard, T.C. Owen, R.A. Toth, M.D. Smith, Oxygen and carbon isotope ratios in the martian atmosphere. Icarus 192, 396-403 (2007)

R.V. Krishnamurthy, S. Epstein, J.R. Cronin, S. Pizzarello, G.U. Yuen, Isotopic and molecular analyses of hydrocarbons and monocarboxylic acids of the Murchison meteorite. Geochim. Cosmochim. Acta 56(11), 4045-4058 (1992)

Y.P. Langevin, F. Poulet, J. Bibring, B. Gondet, Sulfates in the North polar region of Mars detected by OMEGA/Mars Express. Science 307, 1584-1586 (2006)

L. Leshin, Insights into martian water reservoirs from analyses of martian meteorite QUE 94201. Geophys. Res. Lett. 27, 2017-2020 (2000)

G.V. Levin, P.A. Straat, Viking labeled release biology experiment—interim results. Science 194, 1322-1329 (1976)

G.V. Levin, P.A. Straat, Recent results from the Viking Labeled Release experiment on Mars. J. Geophys. Res. 82, 4663-4667 (1977)

G.V. Levin, P.A. Straat, A search for a nonbiological explanation of the Viking Labeled Release life detection experiment. Icarus 45, 494-516 (1981)

W.C. Maguire, Martian isotopic ratios and upper limits for possible minor constituents as derived from Mariner 9 infrared spectrometer data. Icarus 32, 85-97 (1977)

P.R. Mahaffy, Sample analysis at Mars: developing analytical tools to search for a habitable environment on the Red Planet. Geochem. News 121 (2009)

P.R. Mahaffy, D.P. Glavin, J.L. Eigenbrode, H. Franz, J. Stern, D.N. Harpold, S. Team, Calibration of the sample analysis at Mars (SAM) instrument suite for the 2011 Mars science laboratory, in Lunar and Planetary Institute Science Conference Abstracts, vol. 41 (2010), p. 2130

L. Maltagliati, D.V. Titov, T.R.S. Encrenaz, R. Melchiorri, F. Forget, H.U. Keller, J.-P. Bibring, Annual survey of water vapor behavior from the OMEGA mapping spectrometer onboard Mars Express. Icarus 213, 480-495 (2011)

R.L. Mancinelli, M. Klovstad, Martian soil and UV radiation: microbial viability assessment on spacecraft surfaces. Planet. Space Sci. 48, 1093-1097 (2000)

N. Mangold, A. Gendrin, B. Gondet, S. LeMouelic, C. Quantin, V. Ansan, G. Neukum, Spectral and geological study of the sulfate-rich region of West Candor Chasma, Mars. Icarus 194(2), 519-543 (2008)

K.J. Mathew, K. Marti, Early evolution of martian volatiles: nitrogen and noble gas components in ALH84001 and Chassigny. J. Geophys. Res. 106, 1401-1422 (2001)

A.C. McAdam, I.L. Ten Kate, J.C. Stern, P.R. Mahaffy, D.F. Blake, R.V. Morris, A. Team, Field characterization of the mineralogy and organic chemistry of carbonates from the 2010 Arctic Mars analog Svalbard expedition by evolved gas analysis, in Lunar and Planetary Institute Science Conference Abstracts, vol. 42 (2011), p. 2136

T.M. McCollom, Formation of meteorite hydrocarbons from thermal decomposition of siderite $\left(\mathrm{FeCO}_{3}\right)$. Geochim. Cosmochim. Acta 67(2), 311-317 (2003)

T.M. McCollom, B.R.T. Simoneit, Abiotic formation of hydrocarbons and oxygenated compounds during thermal decomposition of iron oxalate. Orig. Life Evol. Biosph. 29, 167-186 (1999)

T.B. McCord, M.L. Nelson, R.N. Clark, A. Meloy, W. Harrison, T.V. Johnson, L. Soderblom, Spectral unit map of Europa. Bull. Am. Astron. Soc. 14, 737 (1982)

F.M. McCubbin, N.J. Tosca, A. Smirnov, H. Nekvasil, A. Steele, M. Fries, D.H. Lindsley, Hydrothermal jarosite and hematite in a pyroxene-hosted melt inclusion in martian meteorite Miller Range (MIL) 03346: implications for magmatic-hydrothermal fluids on Mars. Geochim. Cosmochim. Acta 73, 49074917 (2009)

M.B. McElroy, Mars-evolving atmosphere. Science 175(4020), 443 (1972)

M.B. McElroy, Y.L. Yung, A.O. Nier, Isotopic composition of nitrogen-implications for past history of Mars atmosphere. Science 194(4260), 70-72 (1976)

A.S. McEwen, L. Ojha, C.M. Dundas, S.S. Mattson, S. Byrne, J.J. Wray, S.C. Cull, S.L. Murchie, N. Thomas, C. Gulick, Seasonal flows on warm martian slopes. Science 333(6043), 740-743 (2011)

D.S. McKay, E.K. Gibson, K.L. ThomasKeprta, H. Vali, C.S. Romanek, S.J. Clemett, R.N. Zare, Search for past life on Mars: possible relic biogenic activity in martian meteorite ALH84001. Science 273(5277), 924-930 (1996)

H.Y. McSween, I.O. McGlynn, A.D. Rogers, Determining the modal mineralogy of martian soils. J. Geophys. Res. 115, E00F12 (2010)

U.J. Meierhenrich, W.H.-P. Thiemann, G.M. Muwz Caro, W.A. Schutte, J.M. Greenberg, Simulated cometary matter as a test for enantiomer separating chromatography for use on comet 46P/Wirtanen. Adv. Space Res. 27, 329-334 (2001)

H.J. Melosh, A.M. Vickery, Impact erosion of the primordial atmosphere of Mars. Nature 338(6215), 487489 (1989) 
D. Meunier, R. Sternberg, F. Mettetal, A. Buch, D. Coscia, C. Szopa, F. Raulin, A laboratory pilot for in situ analysis of refractory organic matter in martian soil by gas chromatography-mass spectrometry. Adv. Space Res. 39(3), 337-344 (2007)

J.R. Michalski, P.B. Niles, Deep crustal carbonate rocks exposed by meteor impact on Mars. AGU Fall Meet. Abstr. 44, 04 (2010)

T.J. Millar, Organic molecules in the interstellar medium. Astrobiology 305, 17-31 (2004)

R.E. Milliken, W.W. Fischer, J.A. Hurowitz, Missing salts on early Mars. Geophys. Res. Lett. 36, 11202 (2009)

R.E. Milliken, J.P. Grotzinger, B.J. Thomson, Paleoclimate of Mars as captured by the stratigraphic record in Gale Crater. Geophys. Res. Lett. 37, L04201 (2010)

R.E. Milliken, J.P. Grotzinger, B.J. Thomson, The paleoclimate of Mars as captured by the stratigraphic record in Gale Crater. Lunar Planet. Sci. XLII(2230), L04201 (2011)

D.W. Ming, R.V. Morris, B.C. Clark, Aqueous alteration on Mars, in The Martian Surface-Composition, Mineralogy, and Physical Properties, ed. by J. Bell III (2008), p. 519

I.G. Mitrofanov, M.L. Litvak, A.S. Kozyrev, A.B. Sanin, V.I. Tret'yakov, V.Y. Grin'kov, R.S. Saunders, Soil water content on Mars as estimated from neutron measurements by the HEND instrument onboard the 2001 Mars Odyssey spacecraft. Sol. Syst. Res. 38(4), 253-265 (2004)

R.K. Mohapatra, S.P. Schwenzer, S. Herrmann, S.V.S. Murty, U. Ott, J.D. Gilmour, Noble gases and nitrogen in martian meteorites Dar al Gani 476, Sayh al Uhaymir 005 and Lewis Cliff 88516: EFA and extra neon. Geochim. Cosmochim. Acta 73, 1505-1522 (2009)

R.V. Morris, S.W. Ruff, R. Gellert, D.W. Ming, R.E. Arvidson, B.C. Clark, S.W. Squyres, Identification of carbonate-rich outcrops on Mars by the Spirit Rover. Science 329, 421 (2010)

M.J. Mumma, G.L. Villanueva, R.E. Novak, T. Hewagama, B.P. Bonev, M.A. DiSanti, M.D. Smith, Strong release of methane on mars in northern summer 2003. Science 323(5917), 1041-1045 (2009)

S. Murchie, L. Roach, F. Seelos, R. Milliken, J. Mustard, R. Arvidson, R. Morris, Evidence for the origin of layered deposits in Candor Chasma, Mars, from mineral composition and hydrologic modeling. J. Geophys. Res. 114 (2009a)

S.L. Murchie, J.F. Mustard, B.L. Ehlmann, R.E. Milliken, J.L. Bishop, N.K. McKeown, J.P. Bibring, A synthesis of martian aqueous mineralogy after 1 Mars year of observations from the Mars Reconnaissance Orbiter. J. Geophys. Res. 114 (2009b)

R. Navarro-Gonzalez, K.F. Navarro, J. de la Rosa, E. Iniguez, P. Molina, L.D. Miranda, C.P. McKay, The limitations on organic detection in Mars-like soils by thermal volatilization-gas chromatography-MS and their implications for the Viking results. Proc. Natl. Acad. Sci. USA 103(44), 16089-16094 (2006)

R. Navarro-Gonzalez, E. Iniguez, J. de la Rosa, C.P. McKay, Characterization of organics, microorganisms, desert soils, and Mars-like soils by thermal volatilization coupled to mass spectrometry and their implications for the search for organics on Mars by Phoenix and future space missions. Astrobiology 9(8), 703-715 (2009)

R. Navarro-Gonzalez, E. Vargas, J. de la Rosa, A.C. Raga, C.P. McKay, Reanalysis of the Viking results suggests perchlorate and organics at midlatitudes on Mars. J. Geophys. Res. 115 (2010)

H.B. Niemann, D.N. Harpold, S.K. Atreya, G.R. Carignan, D.M. Hunten, T.C. Owen, Galileo Probe MassSpectrometer Experiment. Space Sci. Rev. 60(1-4), 111-142 (1992)

A.O. Nier, M.B. Mcelroy, Composition and structure of Mars upper-atmosphere-results from neutral mass spectrometers on Vikings 1 and 2. Trans. Am. Geophys. Union 58(8), 827 (1977)

A.O. Nier, M.B. Mcelroy, Y.L. Yung, Isotopic composition of martian atmosphere. Science 194(4260), 68-70 (1976)

P.B. Niles, W.V. Boynton, J.H. Hoffman, D.W. Ming, D. Hamara, Stable isotope measurements of martian atmospheric $\mathrm{CO}_{2}$ at the Phoenix landing site. Science 329(5997), 1334-1337 (2010)

E.G. Nisbet, N.H. Sleep, The habitat and nature of early life. Nature 409(6823), 1083-1091 (2001)

T. Owen, A. Barnun, Comets, impacts and atmospheres. 2. Isotopes and noble gases. In: Volatiles in the Earth and Solar System, vol. 341, pp. 133-138 (1995a)

T. Owen, A. Barnun, Comets, impacts, and atmospheres. Icarus 116(2), 215-226 (1995b)

T. Owen, A. Barnun, I. Kleinfeld, Possible cometary origin of heavy noble-gases in the atmospheres of Venus, Earth and Mars. Nature 358(6381), 43-46 (1992)

T. Owen, K. Biemann, J.E. Biller, A.L. Lafleur, D.R. Rushneck, D.W. Howarth, The composition of the atmosphere at the surface of Mars. J. Geophys. Res. 82, 4635-4639 (1977)

T. Owen, J.P. Maillard, C. Debergh, B.L. Lutz, Deuterium on Mars-the abundance of Hdo and the value of D/H. Science 240(4860), 1767-1770 (1988)

T. Owen, K. Altwegg, W. Huebner, T. Owen, R. Schulz, The contributions of comets to planets, atmospheres, and life: insights from Cassini-Huygens, Galileo, Giotto, and Inner Planet missions, in Origin and Early Evolution of Comet Nuclei, ed. by H. Balsiger (2009), p. 301 
K.I. Oyama, B.J. Berdahl, The Viking Gas Exchange Experiment results from Chryse and Utopia surface samples. J. Geophys. Res. 82, 4669-4676 (1977)

A.A. Pavlov, Organic degradation by ionizing radiation in the shallow subsurface of Mars. Implications for the search of organic molecules by MSL mission. Geophys. Res. Lett. (2011, submitted)

R.O. Pepin, On the origin and early evolution of terrestrial planet atmospheres and meteoritic volatiles. Icarus 92, 2-79 (1991)

R.O. Pepin, Evolution of the martian atmosphere. Icarus 111, 289-304 (1994)

S. Perrier, J.L. Bertaux, F. Lefèvre, S. Lebonnois, O. Korablev, A. Fedorova, F. Montmessin, Global distribution of total ozone on Mars from SPICAM/MEX UV measurements. J. Geophys. Res. (Planets) 111 (2006)

S. Pizzarello, The chemistry of life's origin: a carbonaceous meteorite perspective. Acc. Chem. Res. 39(4), 231-237 (2006)

R.C. Plumb, R. Tantayanon, M. Libby, W.W. Xu, Chemical model for Viking biology experiments: implications for the composition of the martian regolith. Nature 338, 633-635 (1989)

J. Pollack, T. Roush, F. Wittenborn, J. Bregman, D. Wooden, C. Stoker, R. Freedman, Thermal emission spectra of Mars (5.4-10.5 $\mu \mathrm{m})$ : evidence for sulfates, carbonates, and hydrates. J. Geophys. Res. 95, 14559-14627 (1990)

C. Ponnamperuma, A. Shimoyama, M. Yamada, T. Hobo, R. Pal, Possible surface reactions on Mars: implications for Viking biology results. Science 197, 455-457 (1977)

R.C. Quinn, P.J. Grunthaner, C.L. Taylor, C.E. Bryson, F.J. Grunthaner, The radiolytic decomposition of soil perchlorates on Mars, in Lunar and Planetary Institute Science Conference Abstracts, vol. 42 (2011), p. 2003

M.N. Rao, S.R. Sutton, D.S. McKay, G. Dreibus, Clues to Martian brines based on halogens in salts from nakhlites and MER samples. J. Geophys. Res. 110 (2005)

L.H. Roach, J.F. Mustard, S.L. Murchie, J.-P. Bibring, F. Forget, K.W. Lewis, J.L. Bishop, Testing evidence of recent hydration state change in sulfates on Mars. J. Geophys. Res. 114 (2009)

S. Schroder, S.G. Pavlov, I. Rauschenbach, E.K. Jessberger, H.-W. Hübers, Identifying perchlorates under Mars conditions in soil samples and in frozen solutions using LIBS. In: Lunar and Planetary Institute Science Conference Abstracts, vol. 42, p. 1912 (2011)

G. Schwehm, M. Hechler, Rosetta-ESAs Planetary Cornerstone Mission. ESA Bull. 77, 7-18 (1994)

M.A. Sephton, Meteorite composition: organic matter in ancient meteorites. Astron. Geophys. 45, 8 (2004)

M.A. Sephton, I.P. Wright, I. Gilmour, J.W. de Leeuw, M.M. Grady, C.T. Pillinger, High molecular weight organic matter in martian meteorites. Planet. Space Sci. 50, 711-716 (2002)

B. Sherwood Lollar, T.D. Westgate, J.A. Ward, G.F. Slater, G. Lacrampe-Couloume, Abiogenic formation of alkanes in the Earth's crust as a minor source for global hydrocarbon reservoirs. Nature 416, 522-524 (2002)

M.D. Smith, Interannual variability in TES atmospheric observations of Mars during 1999-2003. Icarus 167, 148-165 (2004)

A. Steele, M.D. Fries, H.E.F. Amundsen, B.O. Mysen, M.L. Fogel, M. Schweizer, N.Z. Boctor, Comprehensive imaging and Raman spectroscopy of carbonate globules from martian meteorite ALH 84001 and a terrestrial analogue from Svalbard. Meteorit. Planet. Sci. 42(9), 1549-1566 (2007)

J.C. Stern, A.C. McAdam, I.L. Ten Kate, P.R. Mahaffy, A. Steele, H.E.F. Amundson, $\delta 13 C$ of Mars analog carbonates using evolved gas-Cavity Ringdown Spectrometry on the 2010 Arctic Mars Analog Svalbard Expedition (AMASE), in Lunar and Planetary Institute Science Conference Abstracts, vol. 42 (2011), p. 2403

R.E. Summons, P. Albrecht, G. McDonald, J.M. Moldowan, Molecular biosignatures. Space Sci. Rev. 135, 133-159 (2008)

R.E. Summons, J.P. Amend, D. Bish, R. Buick, G.D. Cody, D.J. Des Marais, D.Y. Sumner, Preservation of martian organic and environmental records: final report of the Mars Biosignature Working Group. Astrobiology 11, 157-181 (2011)

C. Szopa, R. Sternberg, F. Raulin, H. Rosenbauer, What can we expect from the in situ chemical investigation of a cometary nucleus by gas chromatography: first results from laboratory studies. Planet. Space Sci. 51, 863-877 (2003)

L.K. Tamppari, D. Bass, B. Cantor, I. Daubar, C. Dickinson, D. Fisher, M. Wolff, Phoenix and MRO coordinated atmospheric measurements. J. Geophys. Res. 115 (2010)

C.G. Tarsitano, C.R. Webster, Multilaser Herriott cell for planetary tunable laser spectrometers. Appl. Opt. 46(28), 6923-6935 (2007)

F. Tian, J. Kasting, S. Solomon, Thermal escape of carbon from the early martian atmosphere. Geophys. Res. Lett. 36, L02205 (2009)

F. Tian, M.W. Claire, J.D. Haqq-Misra, M. Smith, D.C. Crisp, D. Catling, J.F. Kasting, Photochemical and climate consequences of sulfur outgassing on early Mars. Earth Planet. Sci. Lett. 295(3-4), 412-418 (2010) 
J.S. Toporski, A. Stale From Microbial Follils to Astrobiology (Kluwer, Dordrecht, 2004)

N.J. Tosca, A.H. Knoll, Juvenile chemical sediments and the long term persistence of water at the surface of Mars. Earth Planet. Sci. Lett. 286, 379-386 (2009)

H. Wanke, J. Bruckner, G. Dreibus, R. Rieder, I. Ryabchikov, Chemical composition of rocks and soils at the Pathfinder Site. Space Sci. Rev. 96, 317-330 (2001)

L.L. Watson, I.D. Hutcheon, S. Epstein, E.M. Stolper, Water on Mars-clues from deuterium/hydrogen and water contents of hydrous phases in SNC meteorites. Science 265, 86 (1994)

C.R. Webster, Measuring methane and its isotopes ${ }^{1} 2 \mathrm{CH}_{4},{ }^{1} 3 \mathrm{CH}_{4}$, and $\mathrm{CH}_{3} \mathrm{D}$ on the surface of Mars with in situ laser spectroscopy. Appl. Opt. 44, 1226-1235 (2005)

C.R. Webster, P.R. Mahaffy, Determining the local abundance of martian methane and its, ${ }^{13} \mathrm{C} /{ }^{12} \mathrm{C}$ and $\mathrm{D} / \mathrm{H}$ isotopic ratios for comparison with related gas and soil analysis on the 2011 Mars Science Laboratory (MSL) mission. Planet. Space Sci. 59, 271-283 (2011)

A.S. Wong, S.K. Atreya, T. Encrenaz, Chemical markers of possible hot spots on Mars. J. Geophys. Res. 108, E4 (2003)

I.P. Wright, M.M. Grady, C.T. Pillinger, Chassigny and the nakhlites—carbon-bearing components and their relationship to martian environmental-conditions. Geochim. Cosmochim. Acta 56(2), 817-826 (1992)

A.S. Yen, S.S. Kim, M.H. Hecht, M.S. Frant, B. Murray, Evidence that the reactivity of the martian soil is due to superoxide ions. Science 289, 1909-1912 (2000)

A.S. Yen, D.W. Mittlefehldt, S.M. McLennan, R. Gellert, J.F. Bell, H.Y. McSween, S.W. Squyres, Nickel on Mars: Constraints on meteoritic material at the surface. J. Geophys. Res. 111 (2006)

K. Zahnle, R.S. Freedman, D.C. Catling, Is there methane on Mars? Icarus 212(2), 493-503 (2011)

K. Zahnle, R.M. Haberle, D.C. Catling, J.F. Kasting, Photochemical instability of the ancient Martian atmosphere. J. Geophys. Res. 113, E11 (2008)

A.P. Zent, C.P. McKay, The chemical reactivity of the martian soil and implications for future missions. Icarus 108, 146-157 (1994)

M.Y. Zolotov, E.L. Shock, An abiotic origin for hydrocarbons in the Allan Hills 84001 martian meteorite through cooling of magmatic and impact-generated gases. Meteorit. Planet. Sci. 35(3), 629-638 (2000)

R.W. Zurek, S.E. Smrekar, An overview of the Mars Reconnaissance Orbiter (MRO) science mission. J. Geophys. Res. 112, E5 (2007) 